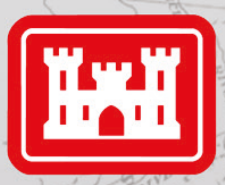

US Army Corps of Engineers ${ }_{\otimes}$

\title{
Bed-Load and Water Surface Measurements during the 2011 Mississippi River Flood at Vicksburg, Mississippi
}

MRG\&P Report No. 18 • April 2018

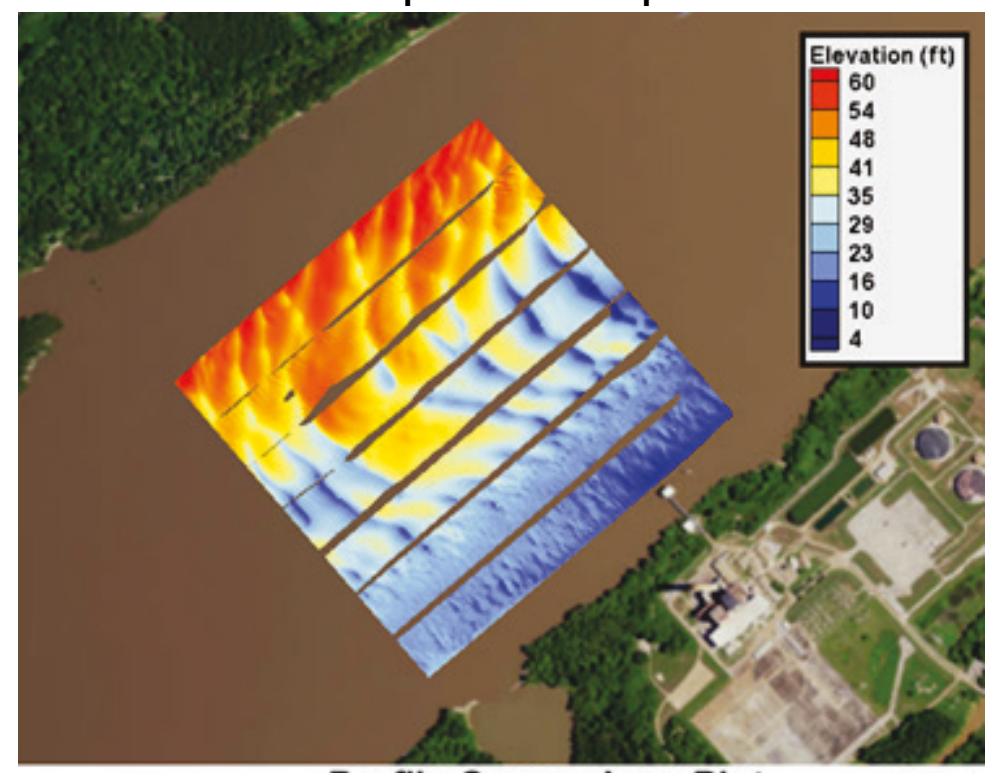

\section{MRG\&P}

Mississippi River Geomorphology \& Potamology Program

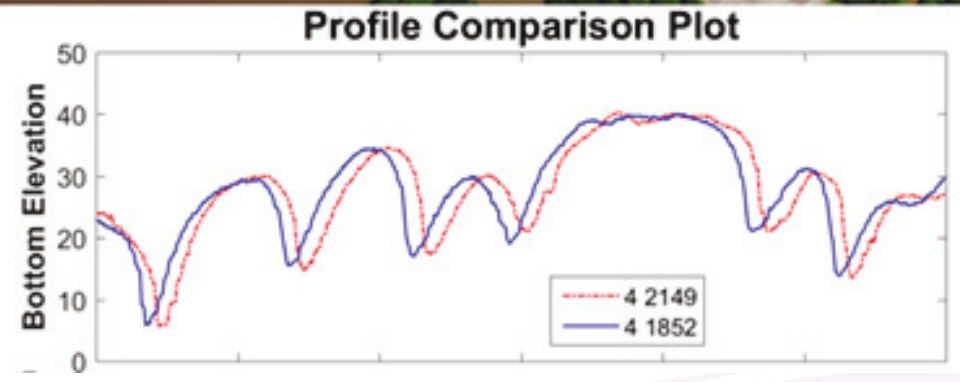




\section{Bed-Load and Water Surface Measurements during the 2011 Mississippi River Flood at Vicksburg, Mississippi}

Keaton E. Jones, David D. Abraham, and Tate O. McAlpin

Coastal and Hydraulics Laboratory

U.S. Army Engineer Research and Development Center

3909 Halls Ferry Road

Vicksburg, MS 39180-6199

Final report

Approved for public release; distribution is unlimited.

Prepared for U.S. Army Corps of Engineers, Mississippi Valley Division Mississippi River Geomorphology \& Potamology Program 1400 Walnut Street

Vicksburg, MS 39180

Under Project 127672, "Bedload Measurement" 


\section{Abstract}

The U.S. Army Engineer Research and Development Center, Coastal and Hydraulics Laboratory, Field Data Collection and Analysis Branch collected bathymetry data and water surface elevations during the 2011 Flood. According to the gage at Vicksburg, MS, the flood peaked at a stage of 57.1 and a flow of 2,310,000 cubic feet per second (cfs) on 17 May 2011, both the highest of record. The reported water surface data include a continuous water surface profile from river mile (RM) 597 to RM 362 and water surface measurements in the vicinity of the Yazoo Backwater levee and Vicksburg, MS. Bathymetry data were also collected and include five sets of surveys, compatible with the Integrated Section Surface Difference over Time Version 2 (ISSDOTv2) method of measuring bed-load transport. These five sets were surveyed at flow rates between 1.6 million and 2.3 million cfs. ISSDOTv2 was used to calculate bed-load transport for the five sets of data. These new bed-load data populate the Lower Mississippi River bed-load rating curve at higher flows and provide a higher level of confidence in the higher-flow portions of the curve. The resulting curve can be a powerful tool for inferring past bed-load transport and predicting future transport.

DISCLAIMER: The contents of this report are not to be used for advertising, publication, or promotional purposes. Citation of trade names does not constitute an official endorsement or approval of the use of such commercial products. All product names and trademarks cited are the property of their respective owners. The findings of this report are not to be construed as an official Department of the Army position unless so designated by other authorized documents. 


\section{Contents}

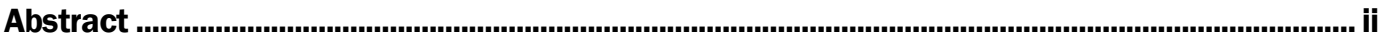

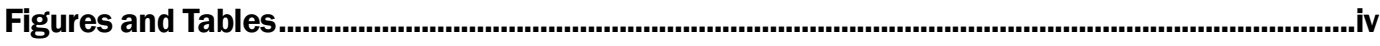

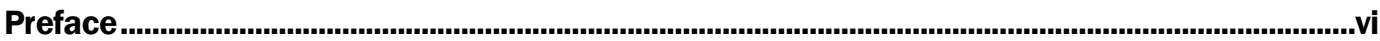
Unit Conversion Factors ................................................................................................................................vii
1 Introduction .................................................................................................................................. 1

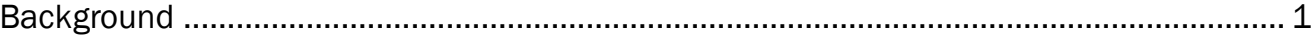

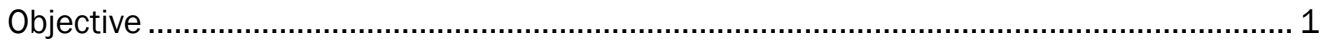

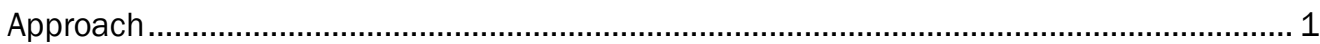

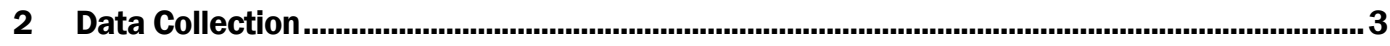

Bathymetry and bed-load transport measurements ....................................................... 3

Water surface elevation ........................................................................................ 7

Water surface profile from Rosedale, MS, to Natchez, MS ................................................... 7

Yazoo Backwater levee water surface elevations ................................................................ 9

Water surface super elevations in vicinity of Vicksburg, MS ............................................... 15

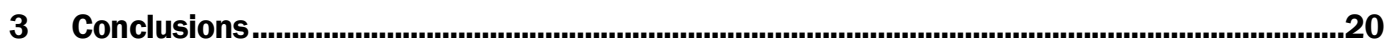

References ................................................................................................................................................22

Appendix A: Dune Survey and Profile Plots ..............................................................................23

Appendix B: Water Surface Profile Data ......................................................................................29

Report Documentation Page 


\section{Figures and Tables}

\section{Figures}

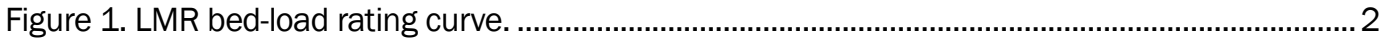

Figure 2. Vicksburg bathymetry measurement locations...................................................................

Figure 3. 13 May 2011 dune field survey................................................................................... 4

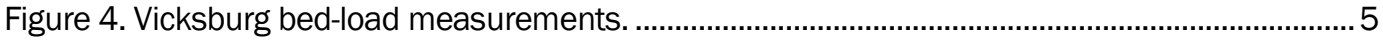

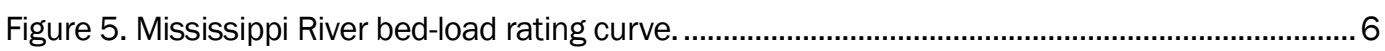

Figure 6. Bed-load delivery at Vicksburg, MS, using the bed-load rating curve for 2009-

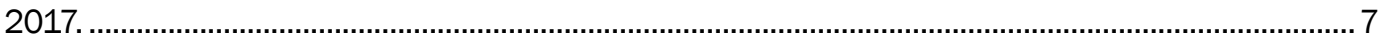

Figure 7. 18 May 2011 water surface elevations. .......................................................................... 8

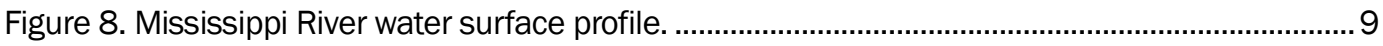

Figure 9. Yazoo Backwater levee water surface elevation survey points and dates...........................10

Figure 10. 06 May 2011 Yazoo Backwater levee water surface measurements (NAVD 88)............12

Figure 11. 06 May 2011 Yazoo Backwater profile (legend indicates time data were

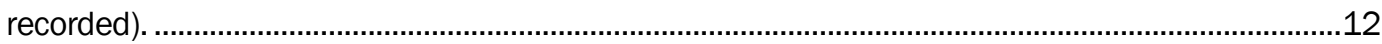

Figure 12. 10 May 2011 Yazoo Backwater levee water surface measurements (NAVD 88)............13

Figure 13. 10 May 2011 Yazoo Backwater profile (legend indicates time data were

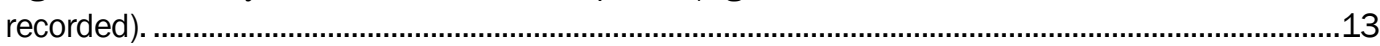

Figure 14. 11 May 2011 Yazoo Backwater levee water surface measurements (NAVD 88)...........14

Figure 15. 11 May 2011 Yazoo Backwater profile. ……………………………………………...14

Figure 16. Spur dike location (image is from 30 July 2015)...........................................................15

Figure 17. 12 May 2011 Vicksburg water surface measurements.....................................................16

Figure 18. 13 May 2011 Vicksburg water surface measurements. ..................................................17

Figure 19. 16 May 2011 Vicksburg water surface measurements. ..................................................18

Figure 20. 15 May 2011 dune field...........................................................................................23

Figure 21. 15 May 2011 example profile. .................................................................................24

Figure 22. 19 May 2011 dune field...............................................................................................2

Figure 23. 19 May 2011 example profile. ..................................................................................2

Figure 24. 26 May 2011 dune field............................................................................................25

Figure 25. 26 May 2011 example profile. ..................................................................................26

Figure 26. 31 May 2011 dune field.................................................................................................26

Figure 27. 31 May 2011 example profile....................................................................................2

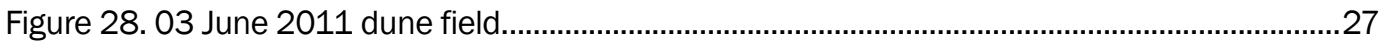

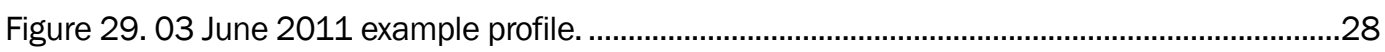

\section{Tables}

Table 1. Vicksburg bed-load measurements........................................................................... 5

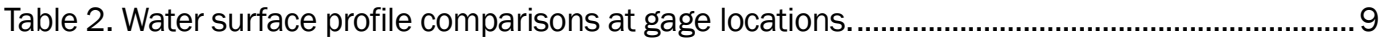

Table 3. Yazoo Backwater levee water surface elevations. ..................................................................10 


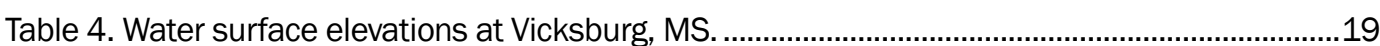

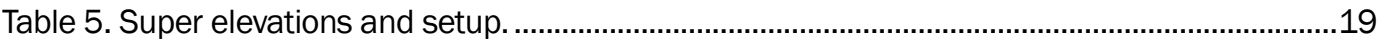




\section{Preface}

The study documented in this report was conducted as part of the Mississippi River Geomorphology and Potamology (MRG\&P) Program; Project 127672, "Bedload Measurement." The MRG\&P is sponsored by Headquarters, U.S. Army Corps of Engineers (USACE), and is managed by the USACE Mississippi Valley Division (MVD) in Vicksburg, MS. The MRG\&P Technical Director was Dr. Ty Wamsley. The MVD Commander was MG Richard G. Kaiser. The MVD Director of Programs was Mr. Jim Bodron.

The Mississippi River Commission provided Mississippi River engineering direction and policy advice. The Commission members were MG Richard G. Kaiser, USACE, President; the Honorable Sam E. Angel; the Honorable Norma Jean Mattei, PhD; RDML Shepard Smith, National Oceanic and Atmospheric Administration; BG Mark Toy, USACE-LRD; and BG Paul E. Owen, USACE-SWD.

At the time of publication, Mr. Jeffrey R. Eckstein was the Acting Director of the U.S. Army Engineer Research and Development Center, Coastal and Hydraulics Laboratory.

COL Bryan S. Green was the Commander of ERDC, and Dr. David W. Pittman was the Director. 


\section{Unit Conversion Factors}

\begin{tabular}{|l|c|l|}
\hline Multiply & By & To Obtain \\
\hline feet & 0.3048 & meters \\
\hline feet & 5280 & miles \\
\hline tons (2,000 pounds, mass) & 907.1847 & kilograms \\
\hline cubic feet & 0.02831685 & cubic meters \\
\hline
\end{tabular}




\section{Introduction}

\section{Background}

The flood on the Mississippi River during the summer of 2011 was a flood of record with 57.1 feet (ft) being read on the Vicksburg gage according to rivergages.com. Measured flow rates at Vicksburg, MS, reached 2.3 million cubic feet per second (cfs) during the flood. Due to the severity and low probability of similar events, the Mississippi Valley Division funded numerous data collection efforts near Vicksburg during this period. The data collected includes the following:

- approximately 2 miles of multi-beam bathymetry collected below the Vicksburg Bridge to determine the ISSDOTv2 bed load measurement site

- five sets of repetitious bathymetric surveys compatible with the ISSDOTv2 bed-load calculation method

- continuous Mississippi River water surface profile from Rosedale, MS, to Natchez, MS (280 miles) on 18 May 2011

- water surface elevations along the Yazoo Backwater levee from the mainline Mississippi River levee to the Steele Bayou Drainage Structure to assist in forecasting backwater levee overtopping potential

- water surface super-elevation measurements at several Mississippi River locations in the vicinity of Vicksburg, MS.

\section{Objective}

The objective of this study is to document the water surface measurements along with bed-load measurements that are calculated from the collected bathymetric data.

\section{Approach}

The approach for calculating bed load transport was to apply the Integrated Section Surface Difference over Time Version 2 (ISSDOTv2) method. ISSDOTv2 is a numerical code that calculates bed-load transport in sand-bed rivers by using multiple bathymetric surveys to track dune scour and deposition. The method is documented in more detail in Abraham et al. (2011). The method has been widely utilized on the Lower 
Mississippi River (LMR) providing a large dataset of measurements (Figure 1). This 2011 data will provide data points at higher flows where there is currently a lack of measurements. The blue dashed lines on Figure 1 represent the flow rates at which the new data were obtained.

Figure 1. LMR bed-load rating curve.

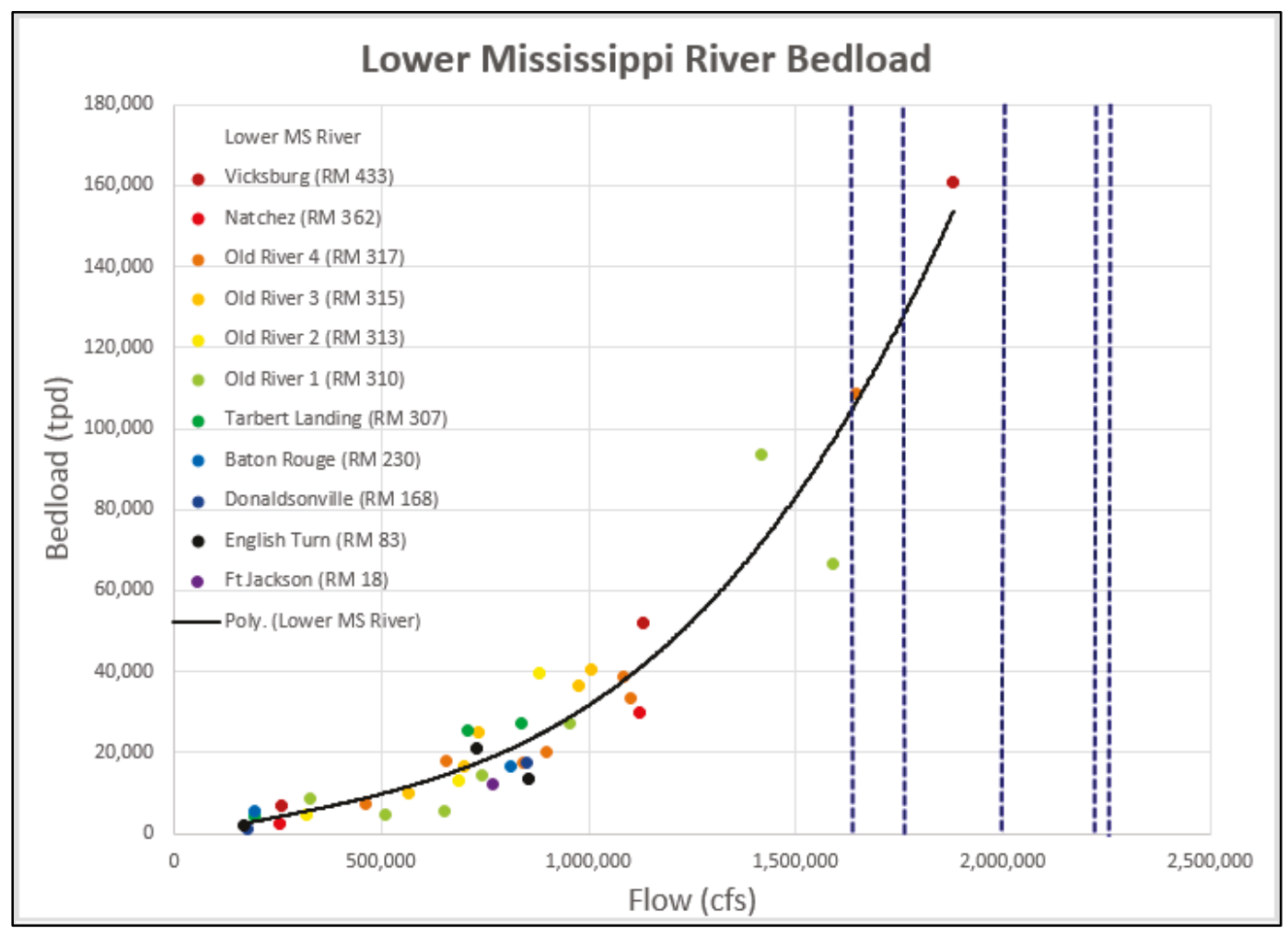




\section{Data Collection}

\section{Bathymetry and bed-load transport measurements}

Approximately 2 miles of multi-beam bathymetry was collected below the Interstate-20 Bridge at Vicksburg, MS, on 13 May 2011 to determine the best location to collect data for the ISSDOTv2 calculations. The location of this 2-mile reach (RM 434.5-432.5) is shown in Figure 2 outlined by the blue box. The data from May 13 are plotted below in Figure 3. The 2 miles contained a consistent formation of dunes along the entire length. Large dunes can be seen in the center of the channel extending towards the right descending bank to the outer extents of the data. Smaller dune formations occur along the thalweg. The ISSDOTv2 method requires sequential surveys to calculate a bed-load transport value, so trips were taken on the following dates near RM 433, which is outlined in red in Figure 2:
1. 16 May 2011
2. 19 May 2011
3. 27 May 2011
4. 31 May 2011
5. 3 Jun 2011.

Figure 2. Vicksburg bathymetry measurement locations.

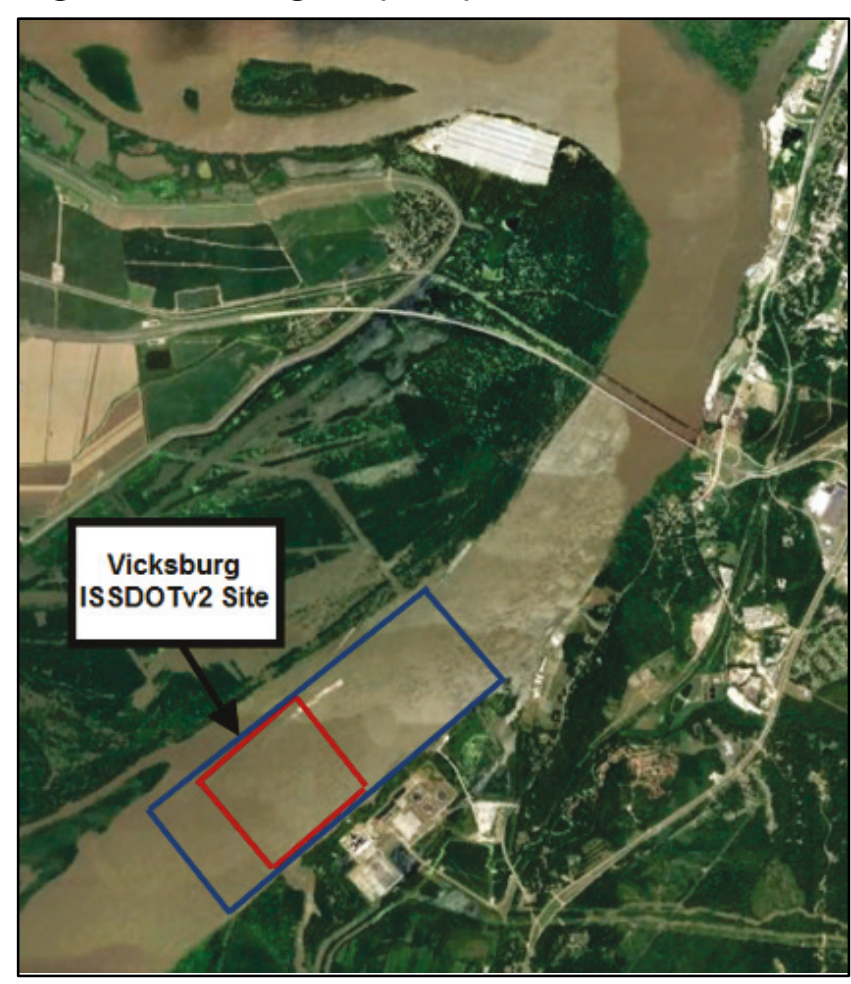


Figure 3. 13 May 2011 dune field survey.

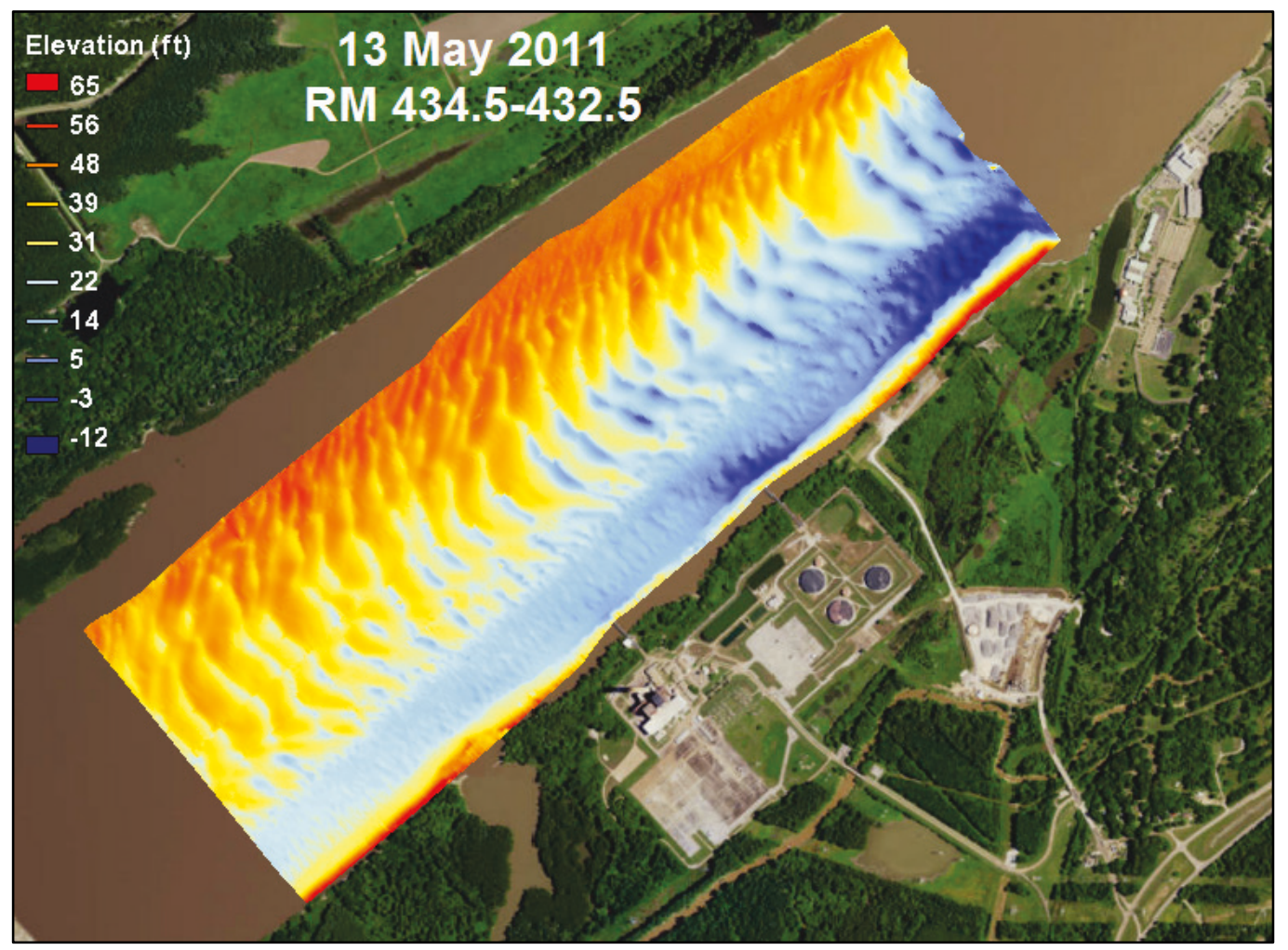

On each trip in 2011, four repeated surveys were taken over approximately a 3- to 4-hour time period. Appendix A includes one set of combined bathymetric surveys and example profiles for each 2011 trip. Results of the 2011 measurements are reported as tons per day (tpd) in Table 1 along with the results of surveys conducted in 2012, 2013, and 2016 at the Vicksburg, MS, site. All eight measurements were computed using the current version of the ISSDOTv2 code to ensure consistency among the results. When combined with the existing measurements, these new datasets produce a rating curve at Vicksburg, MS (Figure 4). These new measurements at Vicksburg, MS, were added to the LMR bed-load curve (Figure 5) providing greater data density at higher flows. The two highest points recorded during a flow of 2,280 and 2,270 thousand cubic feet per second (kcfs) have a difference of 75,000 tpd. There is some variability to be expected, but this large difference shows that flow is not the only dependent factor and that further analysis could help produce a better predictive function. 
Table 1. Vicksburg bed-load measurements.

\begin{tabular}{|c|c|c|}
\hline Date & Flow (kcfs) & Bedload (tpd) \\
\hline 16-May-11 & 2,280 & 277,322 \\
\hline 19-May-11 & 2,270 & 202,509 \\
\hline 26-May-11 & 2,000 & 183,970 \\
\hline 31-May-11 & 1,760 & 118,233 \\
\hline 3-Jun-11 & 1,640 & 113,448 \\
\hline 3-Oct-12 & 261 & 6,936 \\
\hline 29-Apr-13 & 1,132 & 52,047 \\
\hline 15-Jan-16 & 1,880 & 160,947 \\
\hline
\end{tabular}

Figure 4. Vicksburg bed-load measurements.

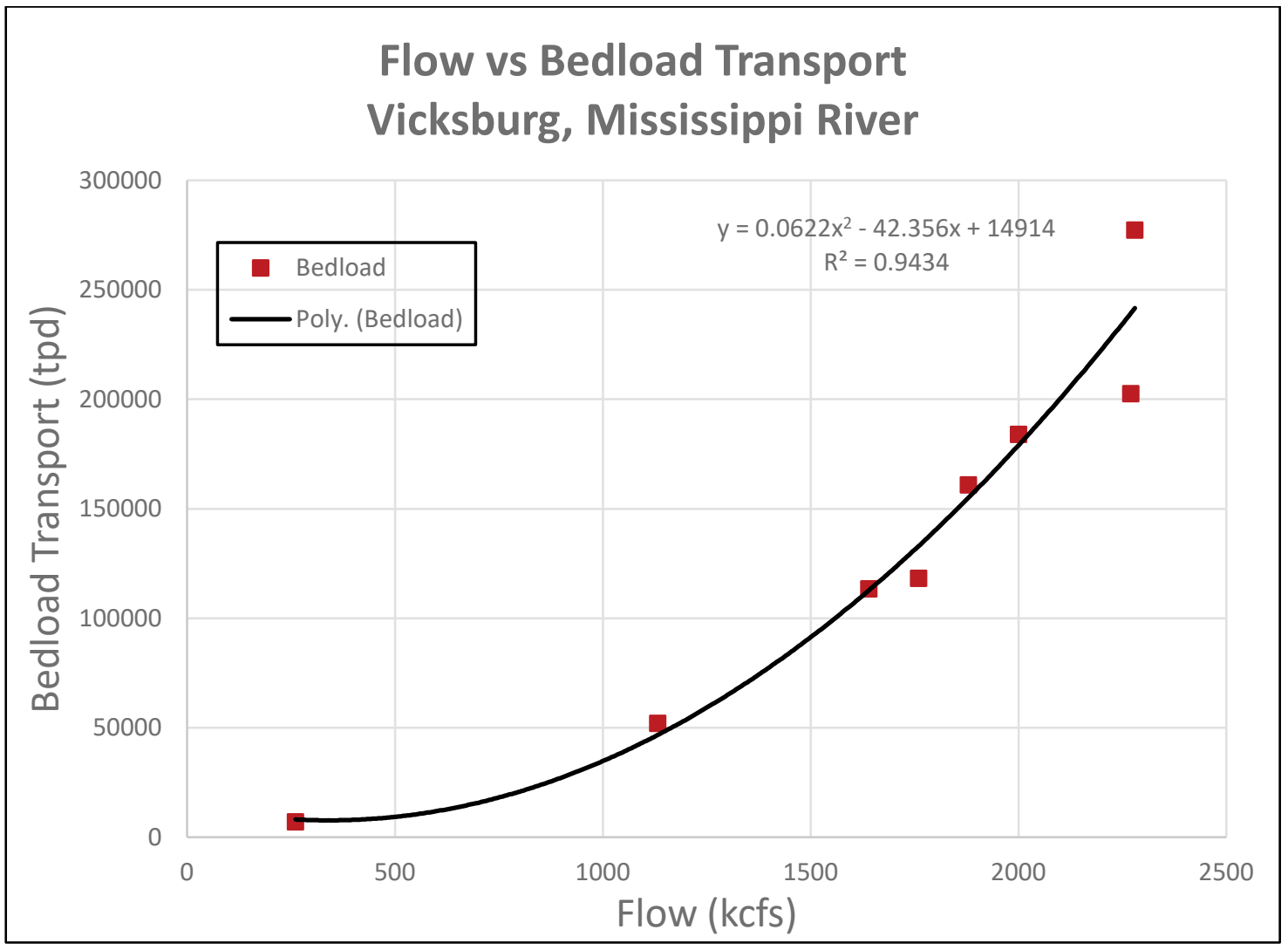


Figure 5. Mississippi River bed-load rating curve.

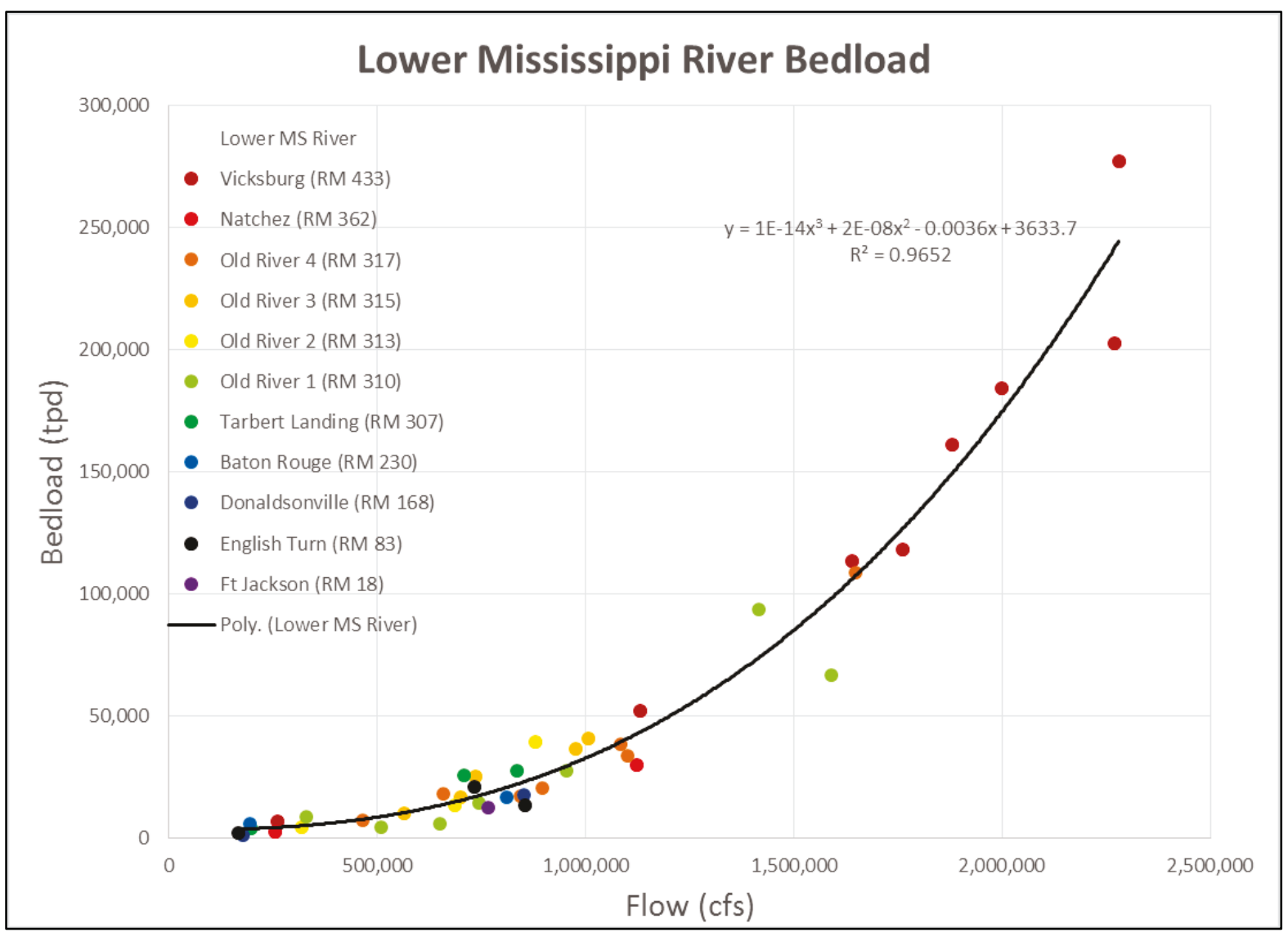

These bed-load rating curves can be used in conjunction with a hydrograph to estimate past sediment delivery at a location. Applying the rating curve to a hydrograph and creating a bed load (tons per day) vs. time curve and then integrating produces a total sediment delivered as bed load for that time period. An example is given below where this method was used to combine the Vicksburg rating curve with yearly hydrographs from the U.S. Geographical Survey (USGS) gage at Vicksburg to calculate total bed load transported past Vicksburg for each water year (October 1September 31) from 2009-2017 (Figure 6). 
Figure 6. Bed-load delivery at Vicksburg, MS, using the bed-load rating curve for 2009-2017.

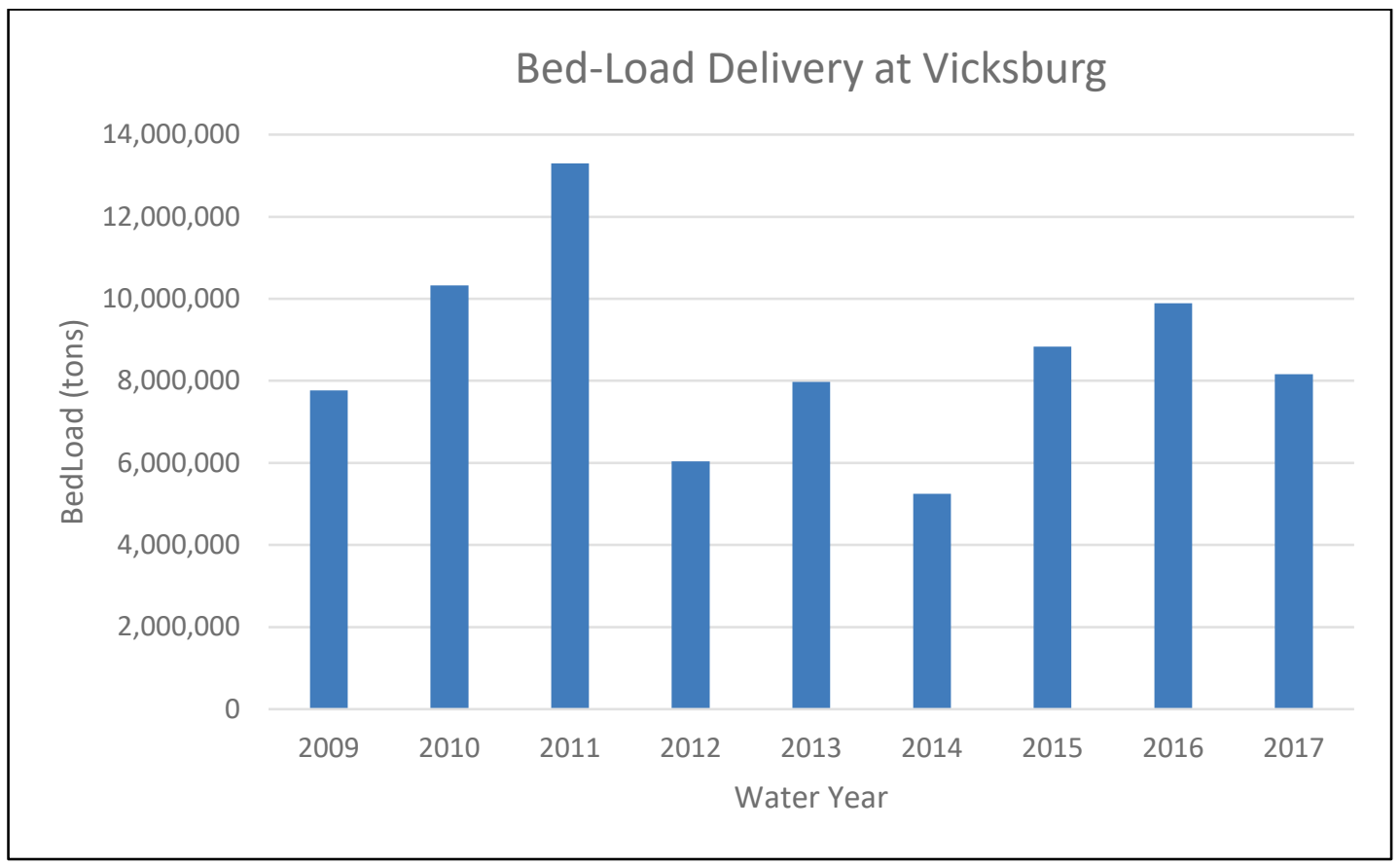

\section{Water surface elevation}

Water surface profile from Rosedale, MS, to Natchez, MS

On 18 May 2011, Coastal and Hydraulics Laboratory (CHL) personnel collected a water surface profile (Figure 7) on the Mississippi River from the mouth of the White River at River Mile 597 to below Natchez, MS, at River Mile 362. The data were collected on May 18 from 1030 to 1800 CDT because the 2011 flood was expected to crest at Vicksburg, MS, that day. The water surface profile with river mile is plotted in Figure 8.

Comparisons were made along the profile at locations where U.S. Army Corps of Engineers gages are located (Table 2). Appendix B includes the data from the profile in tabular form. 
Figure 7. 18 May 2011 water surface elevations.

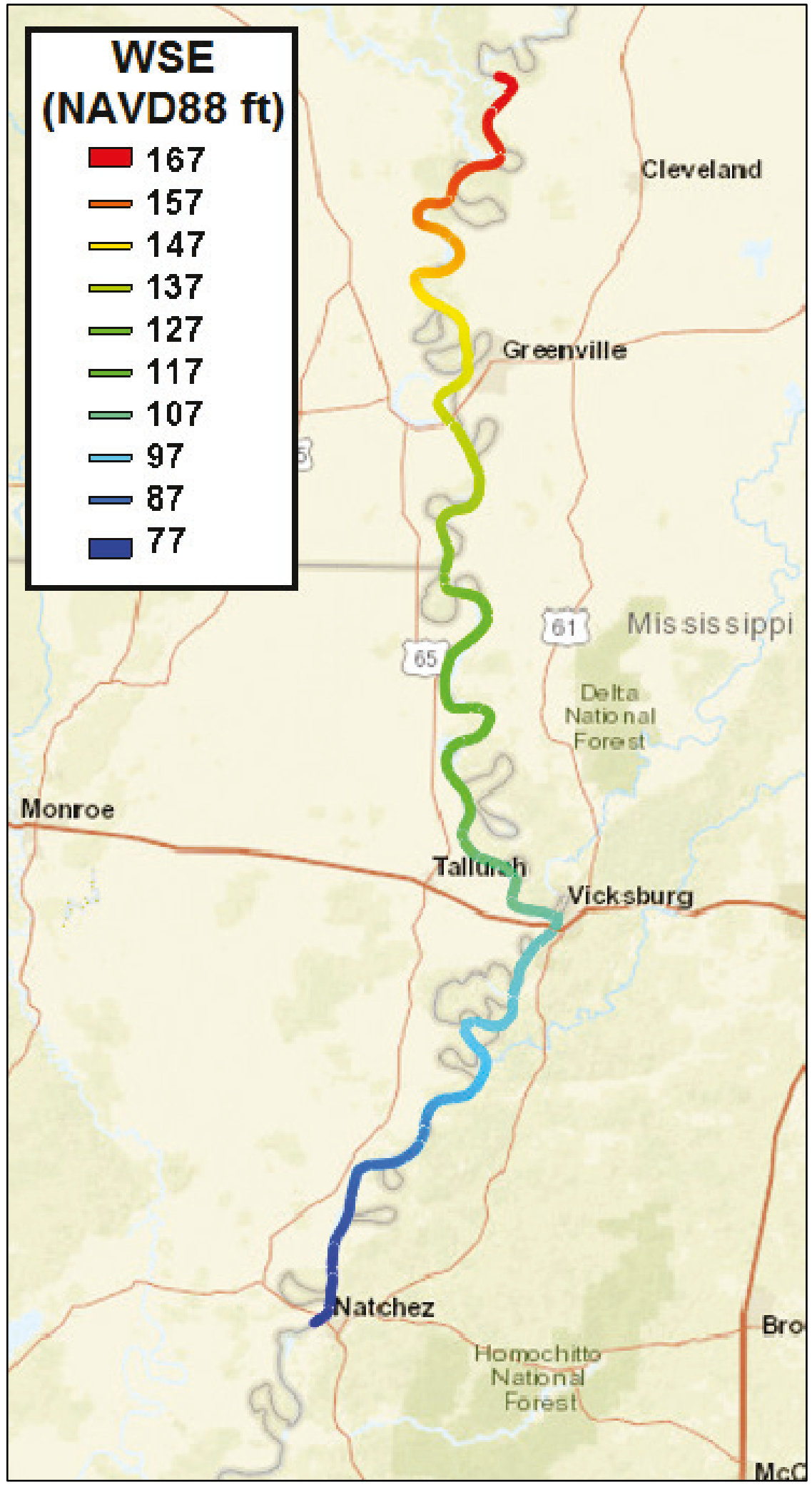


Figure 8. Mississippi River water surface profile.

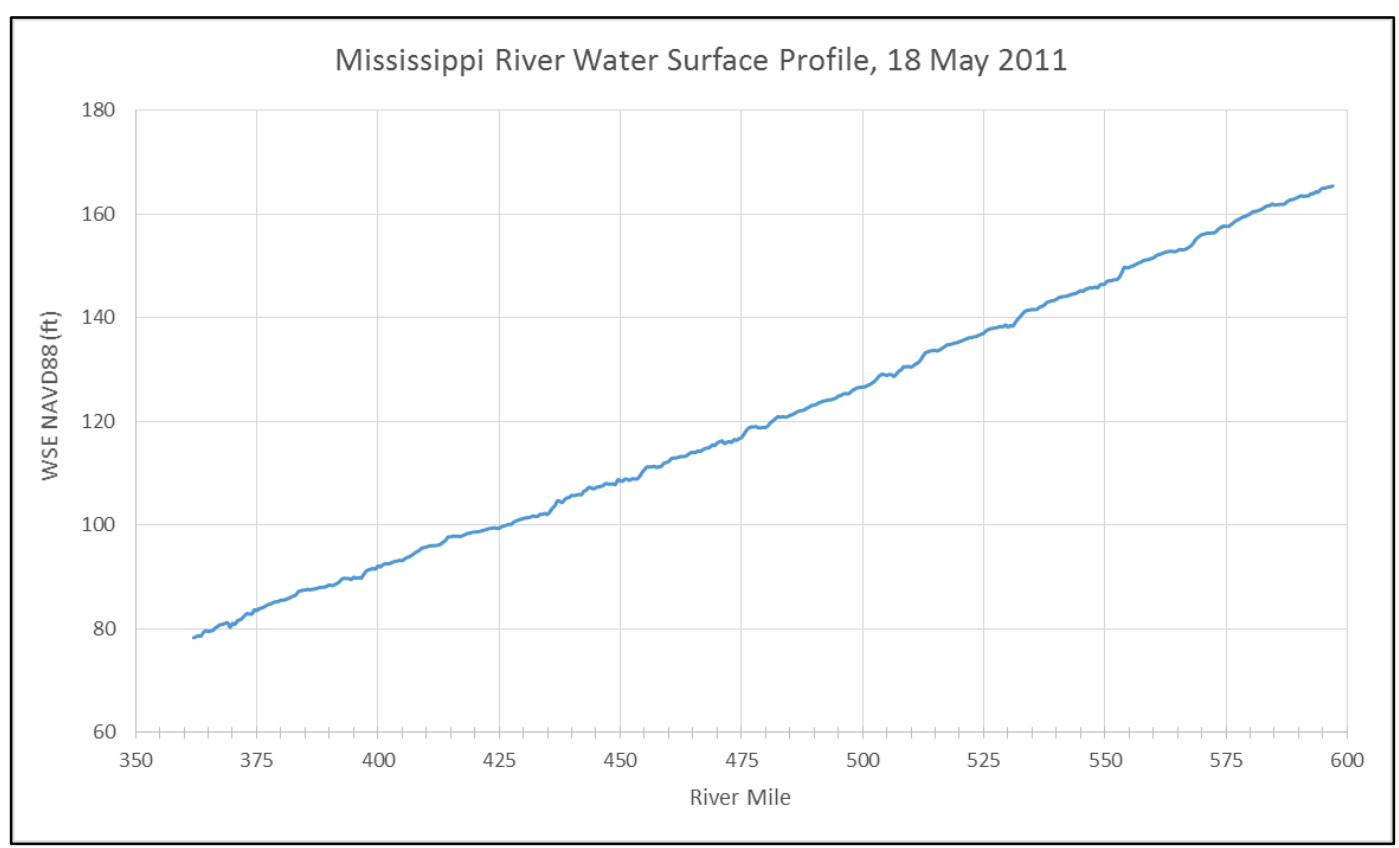

Table 2. Water surface profile comparisons at gage locations.

\begin{tabular}{|c|c|c|c|c|c|}
\hline River Mile & \multicolumn{2}{|c|}{18 May 11 } & \multicolumn{2}{c|}{ From Gage } & CHL Profile \\
\hline & UTC1 & CDT $^{2}$ & NGVD 29 & NAVD 884 & NAVD 88 \\
\hline 553.5 & 1700 & 1200 & 149.44 & 149.05 & 149.1 \\
\hline 531 & 1745 & 1245 & 138.87 & 138.68 & 138.6 \\
\hline 435.7 & 2035 & 1535 & 103.18 & 103.03 & 102.8 \\
\hline 363.3 & 2307 & 1807 & 79.10 & 79.16 & 78.8 \\
\hline
\end{tabular}

${ }^{1}$ Coordinated Universal Time

2 Central Daylight Time

${ }^{3}$ Sea Level Datum of 1929

${ }^{4}$ North American Vertical Datum of 1988

\section{Yazoo Backwater levee water surface elevations}

The CHL Field Data Collection and Analysis Branch took water surface measurements along the Yazoo Backwater levee from the mainline Mississippi River levee up past the Steel Bayou Drainage Structure. These trips took place on the 6th, 10th, and 11th of May 2011. The locations of all survey points and the dates they were collected are depicted in Figure 9. Table 3 summarizes all measurements, and Figure 10 to Figure 15 consist of plan and profile plots of the measurements for each day. 
Figure 9. Yazoo Backwater levee water surface elevation survey points and dates.

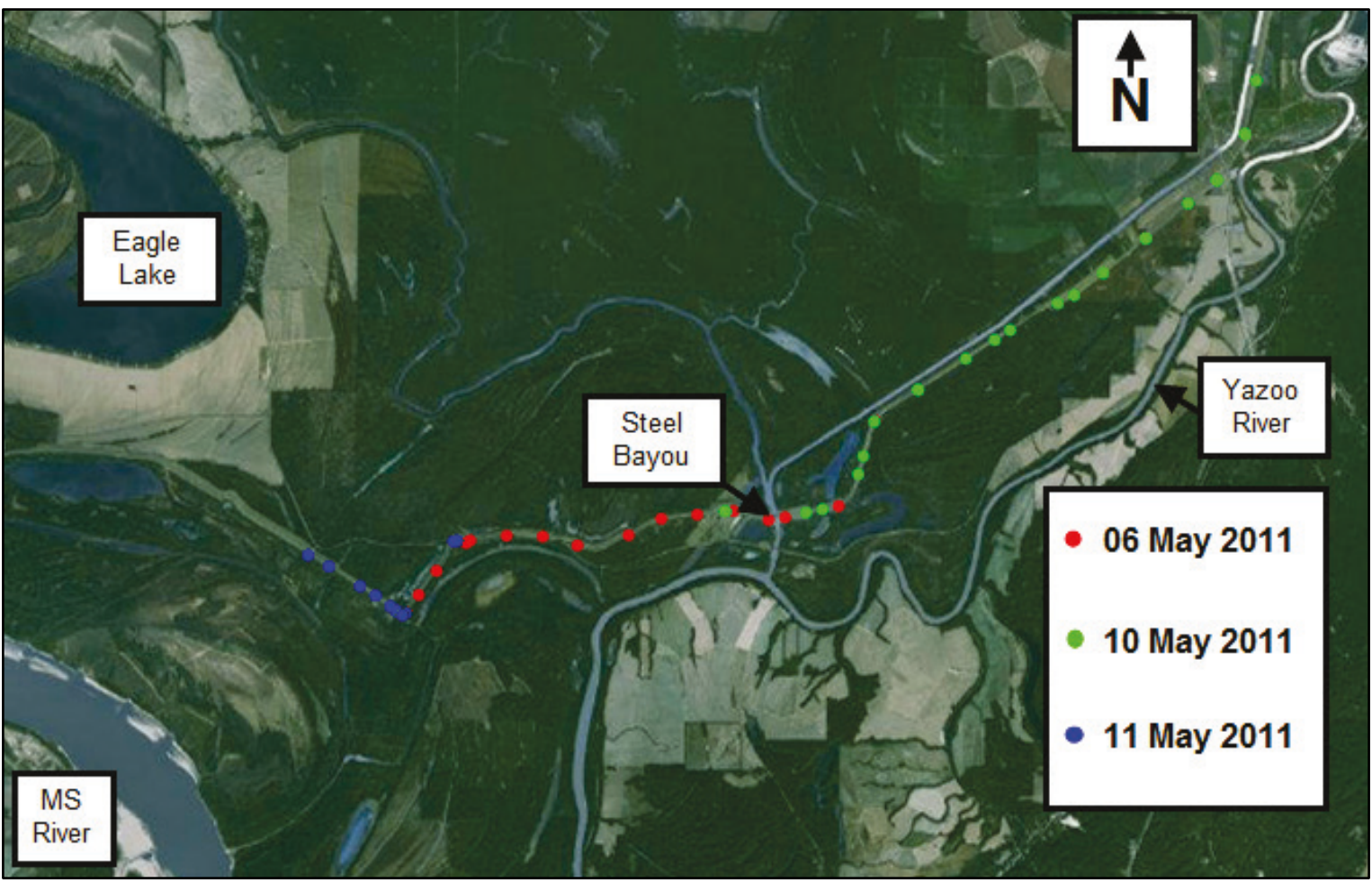

Table 3. Yazoo Backwater levee water surface elevations.

\begin{tabular}{|c|c|c|c|c|}
\hline Date/Time & Northing & Easting & Elevation & Levee Station \\
\hline $5 / 6 / 201115: 00$ & 1070809.98 & 2104618.09 & 98.51 & 0 \\
\hline $5 / 6 / 201115: 06$ & 1071775.80 & 2105288.95 & 98.25 & 1400 \\
\hline $5 / 6 / 201115: 11$ & 1073087.93 & 2106268.42 & 98.17 & 3000 \\
\hline $5 / 6 / 201115: 19$ & 1074614.98 & 2107883.90 & 98.21 & 5700 \\
\hline $5 / 6 / 201115: 26$ & 1074752.46 & 2108110.95 & 98.22 & 6000 \\
\hline $5 / 6 / 201115: 31$ & 1075009.18 & 2110103.75 & 98.34 & 8000 \\
\hline $5 / 6 / 201115: 35$ & 1074964.44 & 2112080.09 & 98.22 & 10000 \\
\hline $5 / 6 / 201115: 40$ & 1074480.07 & 2113971.26 & 98.15 & 12000 \\
\hline $5 / 6 / 201116: 00$ & 1070809.78 & 2104618.23 & 98.48 & 0 \\
\hline $5 / 6 / 201117: 00$ & 1075868.54 & 2124423.53 & 97.84 & 23000 \\
\hline $5 / 6 / 201117: 05$ & 1076358.59 & 2122506.61 & 97.86 & 21000 \\
\hline $5 / 6 / 201117: 09$ & 1076168.30 & 2120522.57 & 97.87 & 19000 \\
\hline $5 / 6 / 201117: 12$ & 1075942.85 & 2118558.99 & 97.92 & 17000 \\
\hline $5 / 6 / 201117: 16$ & 1075036.41 & 2116769.81 & 98.10 & 15000 \\
\hline $5 / 6 / 201117: 20$ & 1074472.18 & 2113985.72 & 98.10 & 12000 \\
\hline $5 / 6 / 201117: 52$ & 1076008.53 & 2125333.95 & 97.86 & 24000 \\
\hline
\end{tabular}




\begin{tabular}{|c|c|c|c|c|}
\hline Date/Time & Northing & Easting & Elevation & Levee Station \\
\hline 5/6/2011 17:57 & 1076635.43 & 2128258.84 & 97.84 & 27000 \\
\hline 5/6/2011 18:00 & 1081292.16 & 2130234.80 & 97.95 & 32000 \\
\hline 5/6/2011 18:04 & 1084144.99 & 2134411.98 & 97.82 & 37000 \\
\hline 5/10/2011 11:40 & 1076026.15 & 2125287.20 & 101.90 & 24000 \\
\hline 5/10/2011 11:45 & 1076452.64 & 2127366.09 & 101.87 & 26000 \\
\hline 5/10/2011 11:52 & 1078373.56 & 2129327.53 & 101.85 & 29000 \\
\hline 5/10/2011 11:53 & 1081243.90 & 2130198.65 & 101.90 & 32000 \\
\hline 5/10/2011 11:58 & 1082987.72 & 2132607.47 & 101.80 & 35000 \\
\hline 5/10/2011 12:06 & 1084682.64 & 2135213.07 & 101.84 & 38000 \\
\hline 5/10/2011 12:11 & 1086241.75 & 2137635.88 & 101.85 & 41000 \\
\hline $5 / 10 / 2011$ 12:20 & 1087697.41 & 2140242.72 & 101.94 & 44000 \\
\hline $5 / 10 / 201112: 24$ & 1089360.46 & 2142727.75 & 101.86 & 47000 \\
\hline 5/10/2011 12:30 & 1091256.51 & 2145047.75 & 101.87 & 50000 \\
\hline 5/10/2011 12:37 & 1093160.18 & 2147370.86 & 101.77 & 53000 \\
\hline $5 / 10 / 2011$ 12:42 & 1094440.50 & 2148931.93 & 101.82 & 55000 \\
\hline 5/10/2011 13:15 & 1096943.45 & 2150490.48 & 101.70 & 58000 \\
\hline 5/10/2011 13:22 & 1099869.70 & 2151094.85 & 101.59 & 61000 \\
\hline $5 / 10 / 2011$ 14:41 & 1096947.84 & 2150491.08 & 101.80 & 58000 \\
\hline $5 / 10 / 2011$ 14:48 & 1094440.98 & 2148931.63 & 101.91 & 55000 \\
\hline $5 / 10 / 2011$ 14:53 & 1091256.86 & 2145047.81 & 101.99 & 50000 \\
\hline 5/10/2011 14:57 & 1088141.34 & 2141133.92 & 101.91 & 45000 \\
\hline 5/10/2011 15:02 & 1085702.92 & 2136795.25 & 102.03 & 40000 \\
\hline 5/10/2011 15:06 & 1082975.70 & 2132589.52 & 101.97 & 35000 \\
\hline 5/10/2011 15:12 & 1079355.72 & 2129602.14 & 101.96 & 30000 \\
\hline 5/10/2011 15:17 & 1076279.33 & 2126471.98 & 101.88 & 25000 \\
\hline 5/10/2011 15:24 & 1076350.05 & 2122042.40 & 101.91 & 20500 \\
\hline $5 / 11 / 2011$ 12:46 & 1073951.71 & 2099242.82 & 105.21 & 61700 \\
\hline $5 / 11 / 201112: 56$ & 1073323.00 & 2100390.43 & 105.25 & 63000 \\
\hline 5/11/2011 13:02 & 1072257.31 & 2102081.93 & 104.96 & 65000 \\
\hline 5/11/2011 13:06 & 1071743.12 & 2102927.38 & 104.84 & 66000 \\
\hline 5/11/2011 13:10 & 1071151.22 & 2103737.31 & 104.75 & 67000 \\
\hline 5/11/2011 13:17 & 1070997.08 & 2103952.21 & 104.73 & 67300 \\
\hline 5/11/2011 13:21 & 1070864.76 & 2104139.77 & 104.75 & 67500 \\
\hline
\end{tabular}




\begin{tabular}{|c|c|c|c|c|}
\hline Date/Time & Northing & Easting & Elevation & Levee Station \\
\hline $5 / 11 / 201113: 50$ & 1070673.98 & 2104408.05 & 104.78 & 67800 \\
\hline $5 / 11 / 201112: 15$ & 1070672.89 & 2104408.83 & 104.81 & 67800 \\
\hline $5 / 11 / 201113: 45$ & 1070773.39 & 2104572.94 & 103.58 & 200 \\
\hline $5 / 11 / 201112: 06$ & 1070773.48 & 2104573.35 & 103.67 & 200 \\
\hline $5 / 11 / 201114: 38$ & 1074702.74 & 2107187.19 & 103.58 & 5200 \\
\hline $5 / 11 / 201114: 34$ & 1074735.44 & 2107373.99 & 103.46 & 5200 \\
\hline
\end{tabular}

Figure 10. 06 May 2011 Yazoo Backwater levee water surface measurements (NAVD 88).

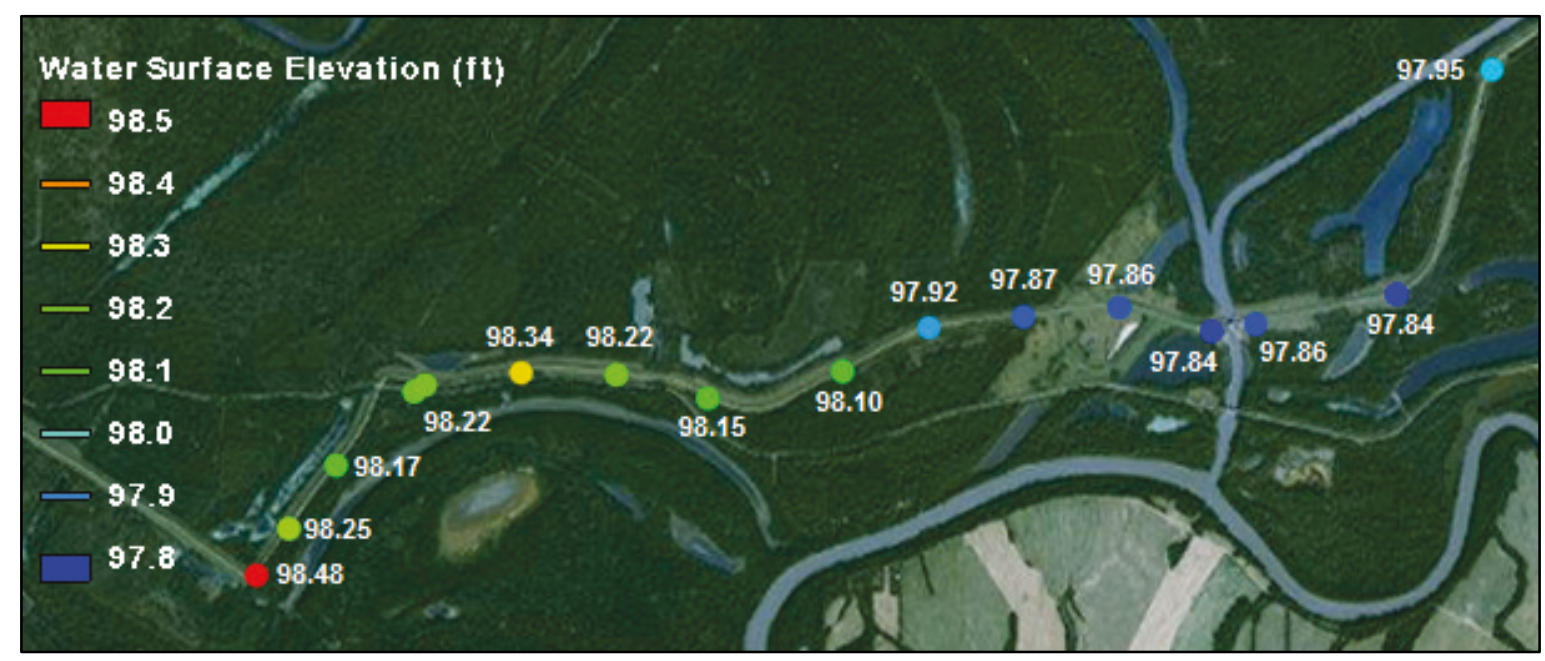

Figure 11. 06 May 2011 Yazoo Backwater profile (legend indicates time data were recorded).

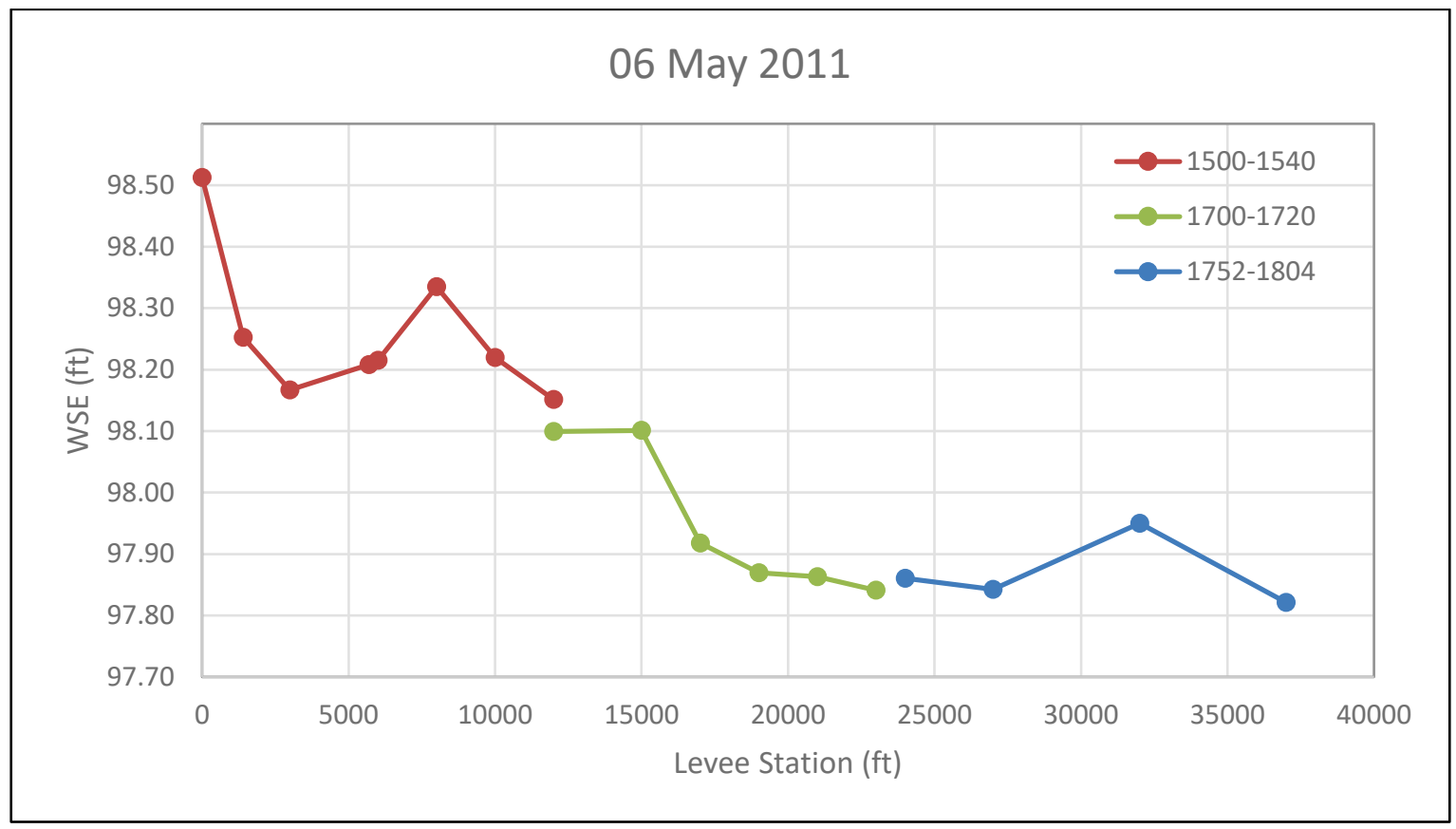


Figure 12. 10 May 2011 Yazoo Backwater levee water surface measurements (NAVD 88).

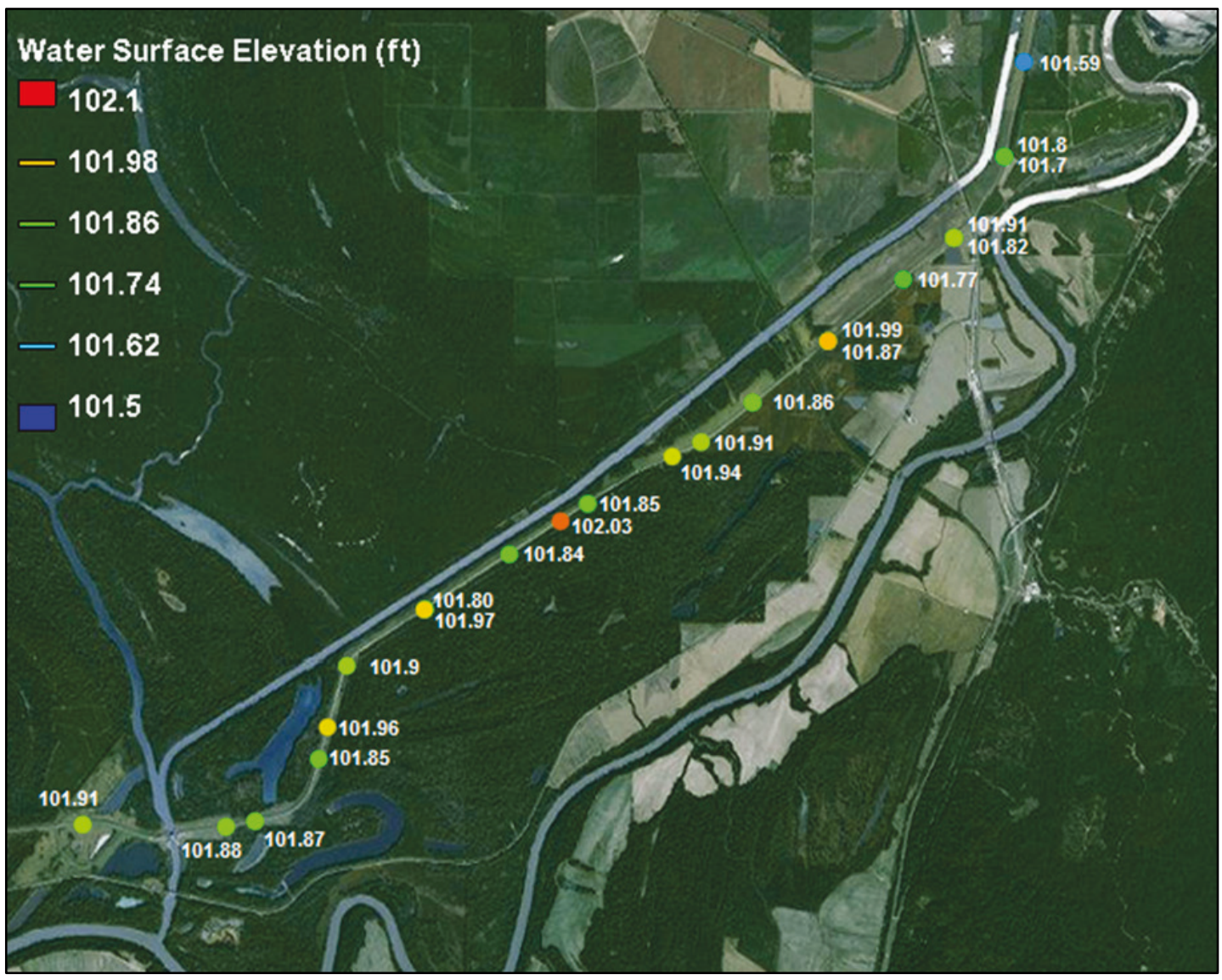

Figure 13. 10 May 2011 Yazoo Backwater profile (legend indicates time data were recorded).

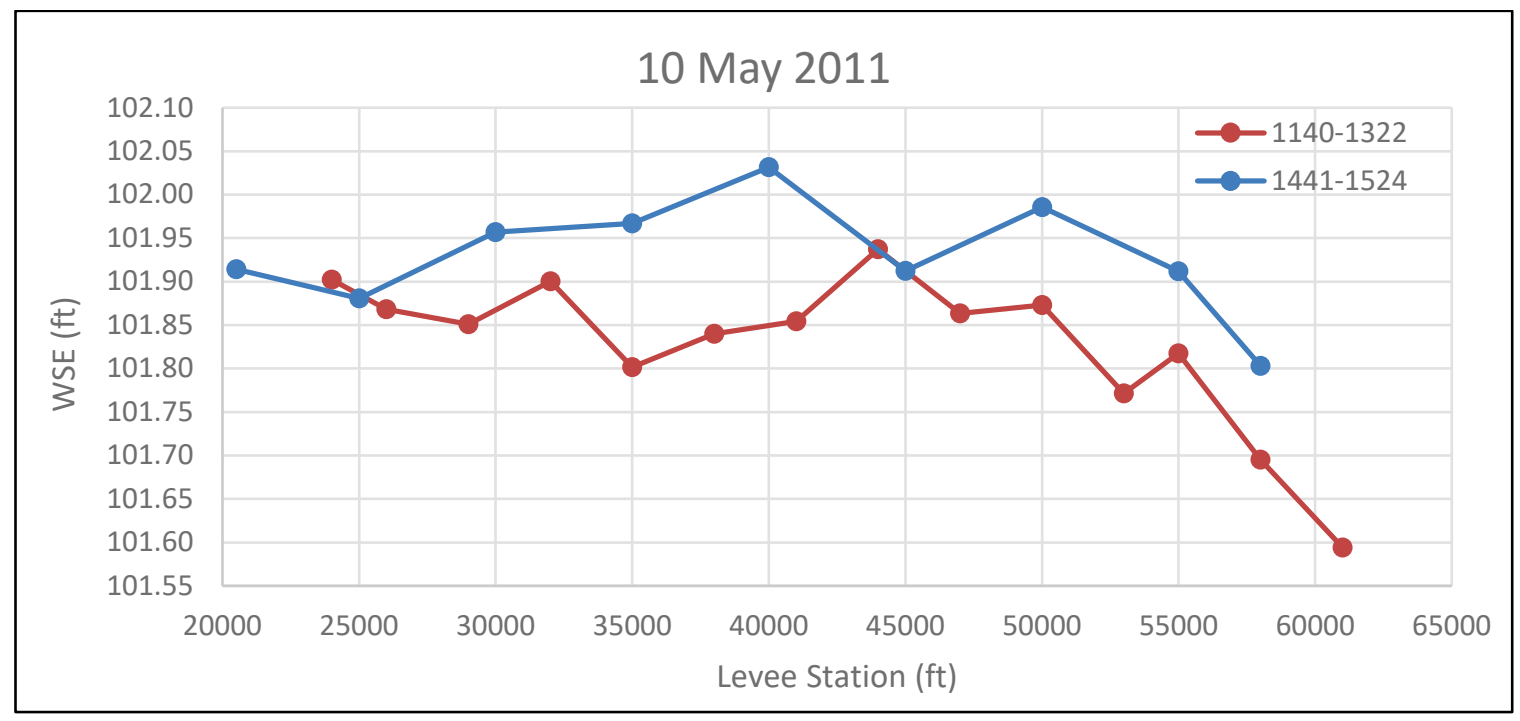


Figure 14. 11 May 2011 Yazoo Backwater levee water surface measurements (NAVD 88).

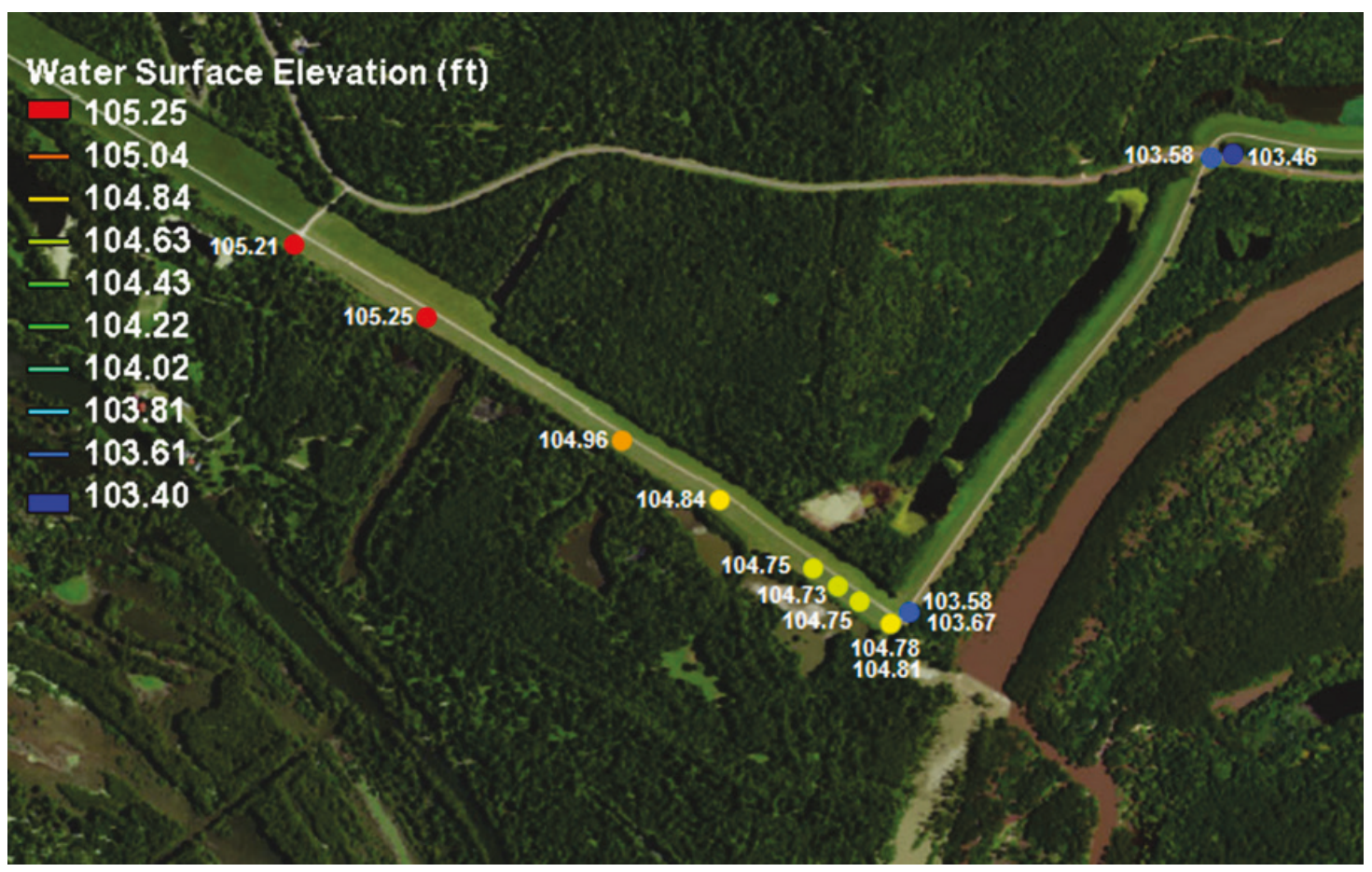

Figure 15. 11 May 2011 Yazoo Backwater profile.

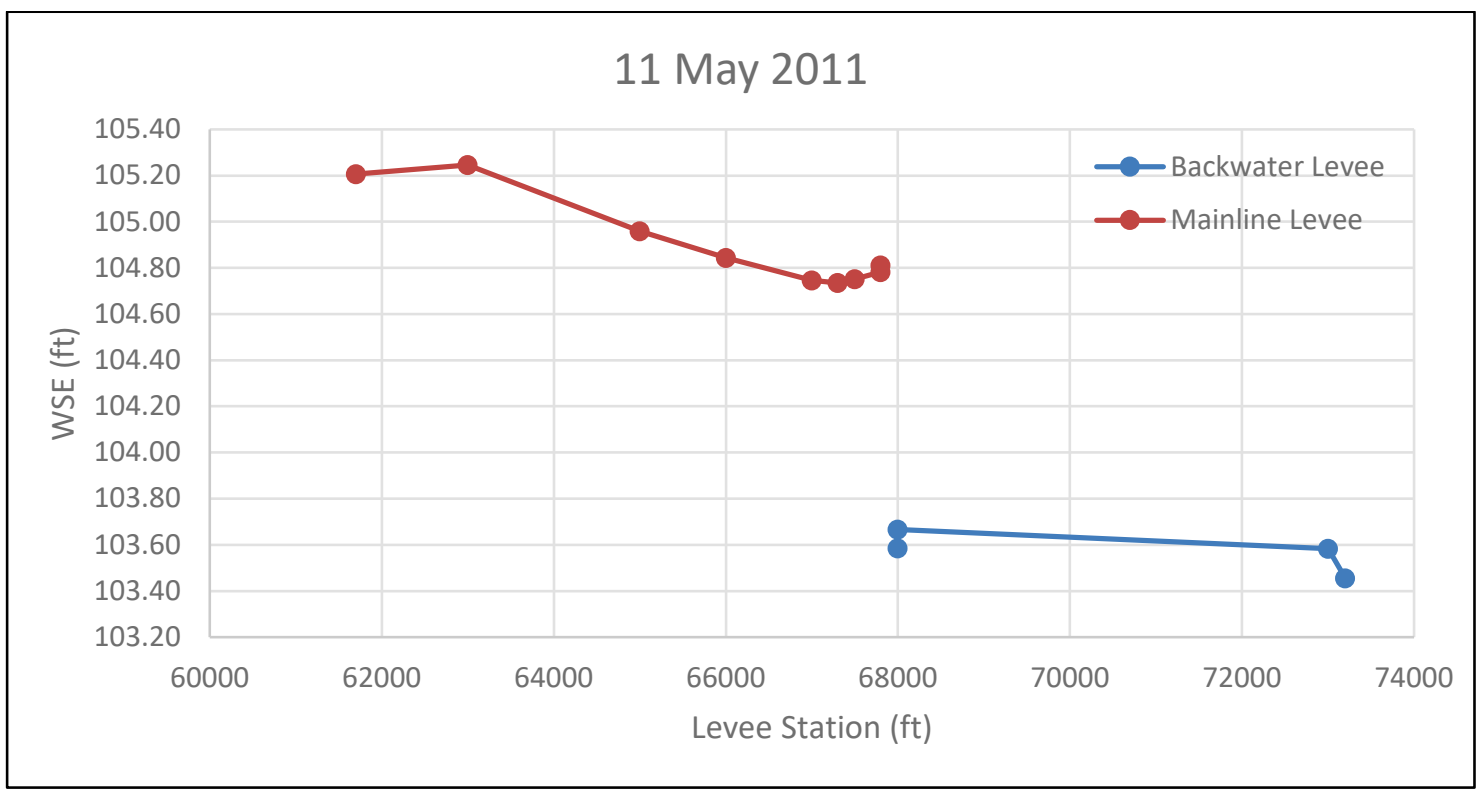

The 11 May 2011 data include measurements on both the mainline levee and the backwater levee. For the water surface profile for this day (Figure 15), the backwater levee stations continue with the mainline levee numbering instead of starting at station zero. For the data collected on 11 May 2011, there is an obvious irregular drop in the profile near station 68,000 $\mathrm{ft}$ where the backwater levee and the mainline levee connect. A member of the 
collection team observed this drawdown in the field. Water was seen rushing over the spur dike (Figure 16) with the downstream water elevation lower than upstream of the dike. The floodwaters during the 2011 Flood would be much higher than this image, which is from 30 July 2015. The Vicksburg gage reading was $11 \mathrm{ft}$ higher during the peak of the flood than it was the date this image was recorded.

Figure 16. Spur dike location (image is from 30 July 2015).

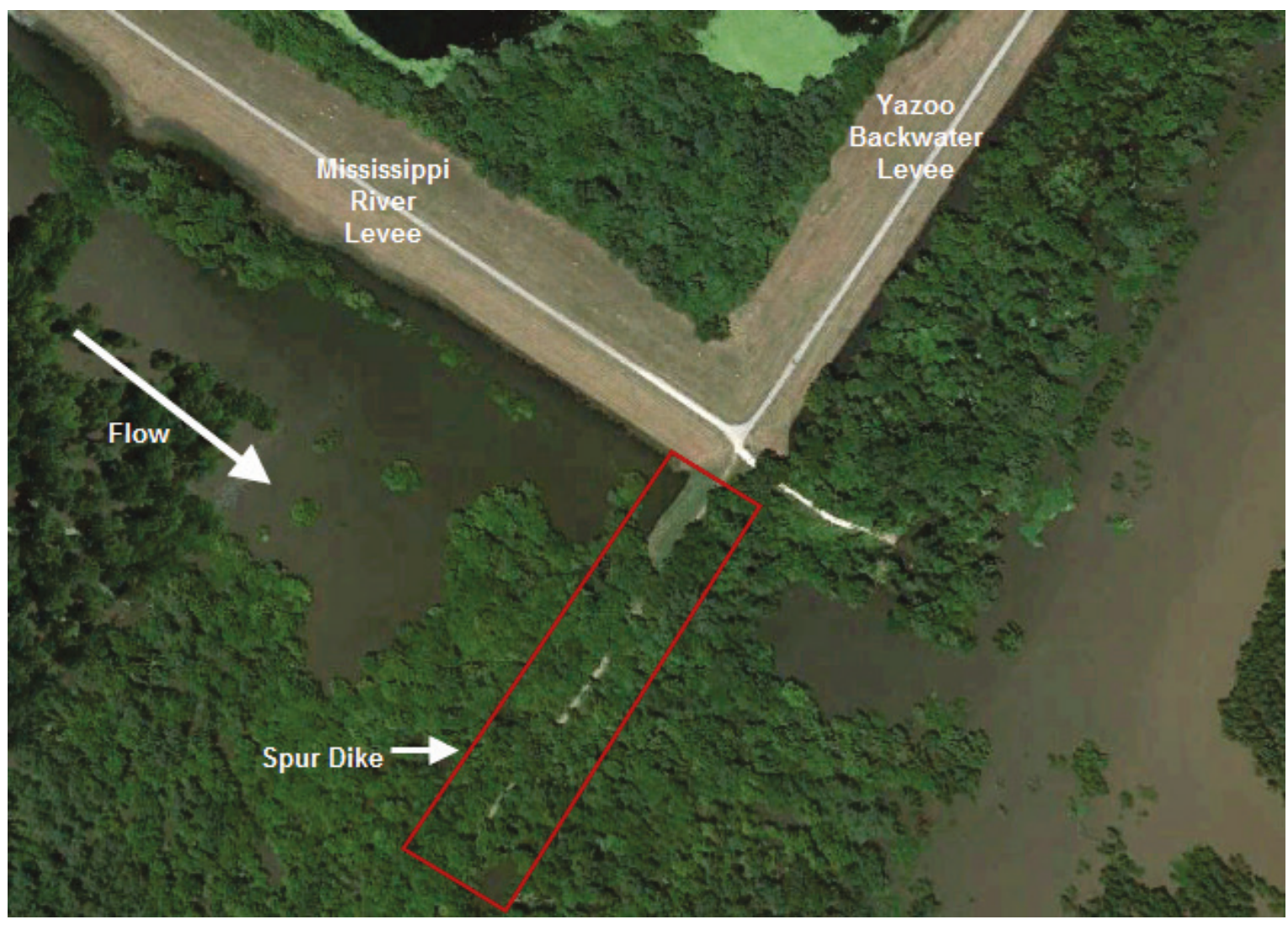

\section{Water surface super elevations in vicinity of Vicksburg, MS}

CHL also measured water surface elevation along the Mississippi River at different locations near Vicksburg, MS. Data were collected the 12th, 13th, and 16th of May 2011. Some of these were super elevation measurements with water levels being recorded on opposite sides of the channel to determine the setup or increase in elevation across the channel. These measurement locations were estimated in the field. The water surface measurements are mapped for each date in Figure 17 to Figure 19 and tabulated in Table 4. Super elevations and corresponding setup are reported in Table 5 . 
Figure 17. 12 May 2011 Vicksburg water surface measurements.

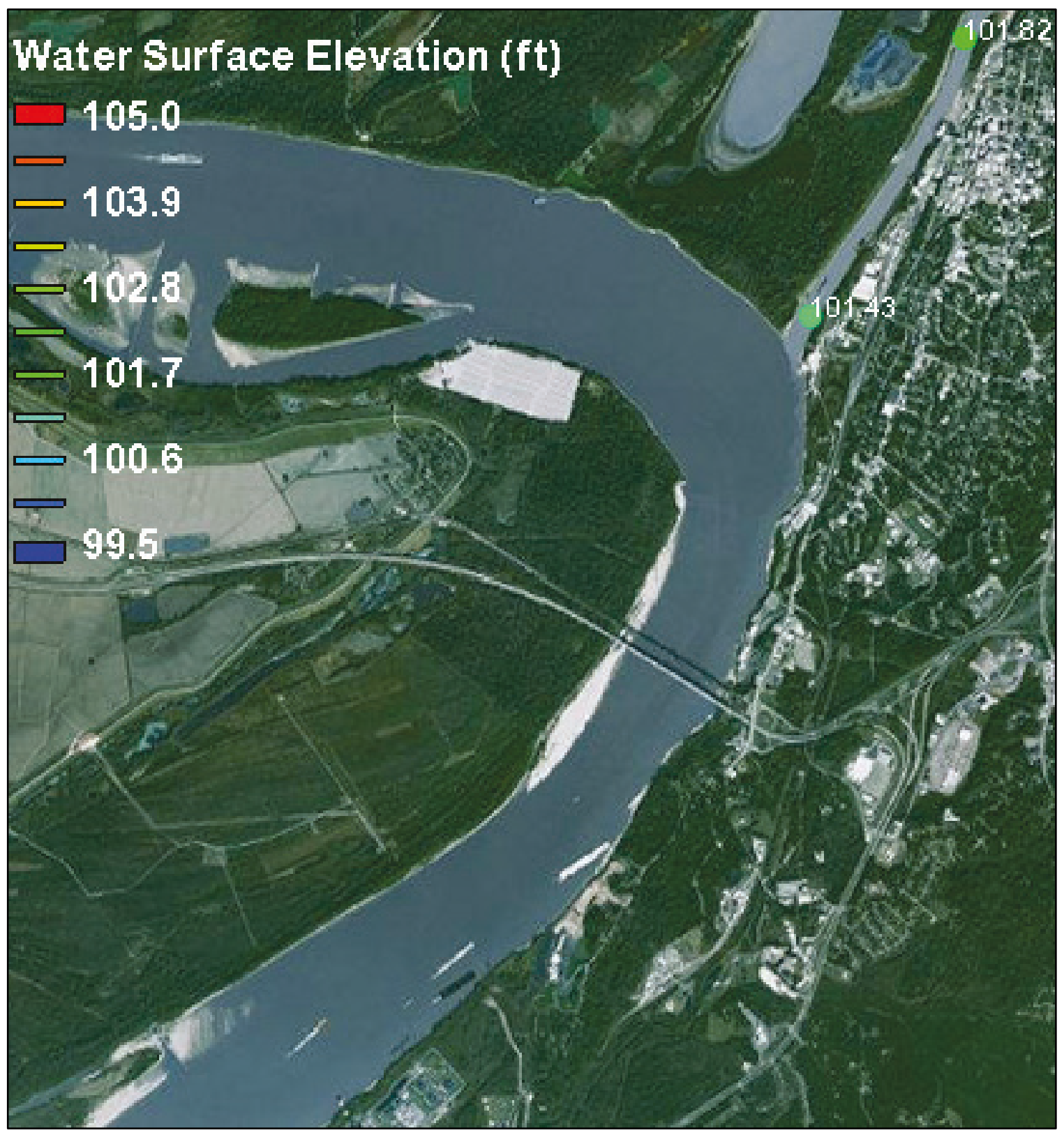


Figure 18. 13 May 2011 Vicksburg water surface measurements.

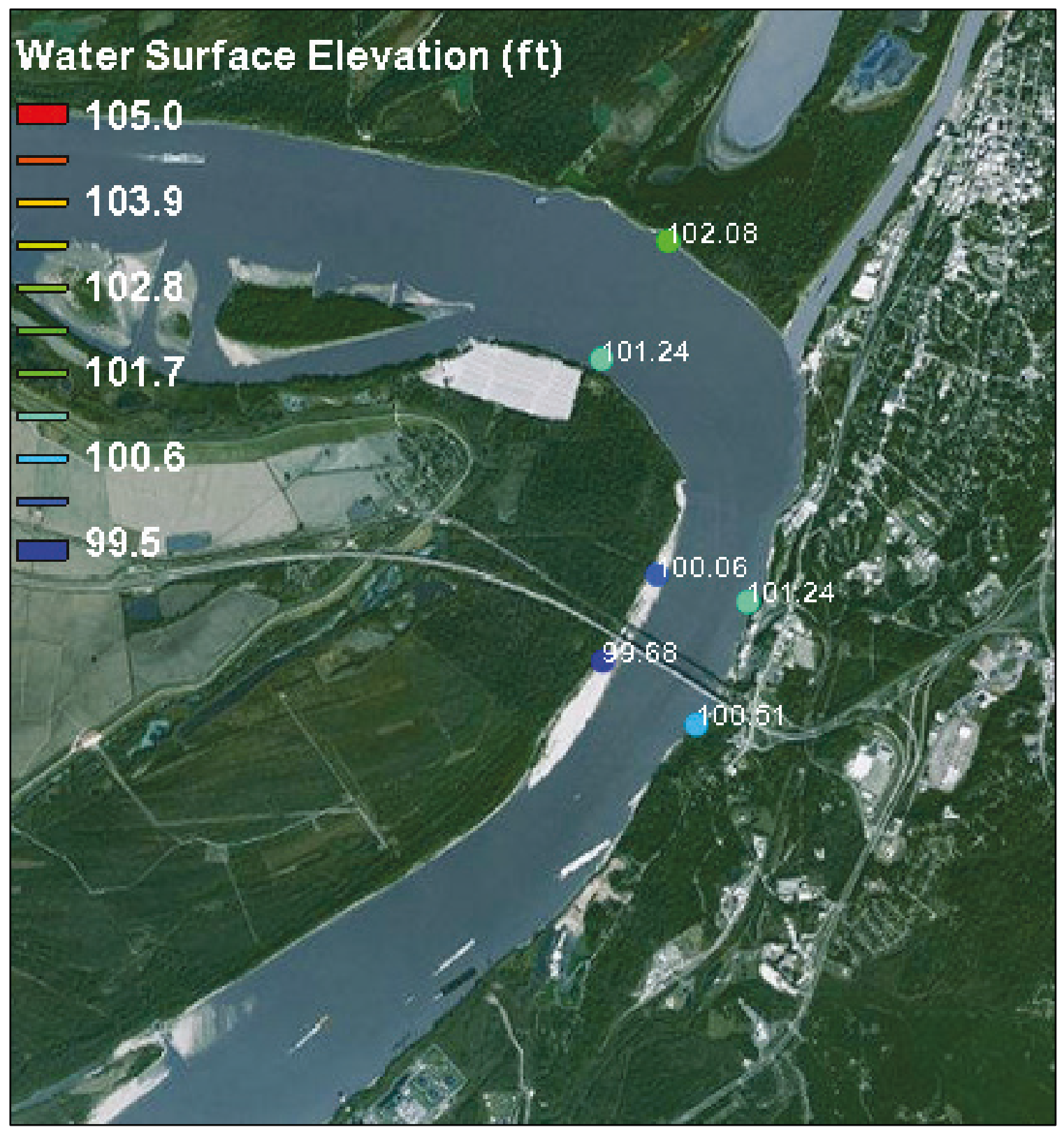


Figure 19. 16 May 2011 Vicksburg water surface measurements.

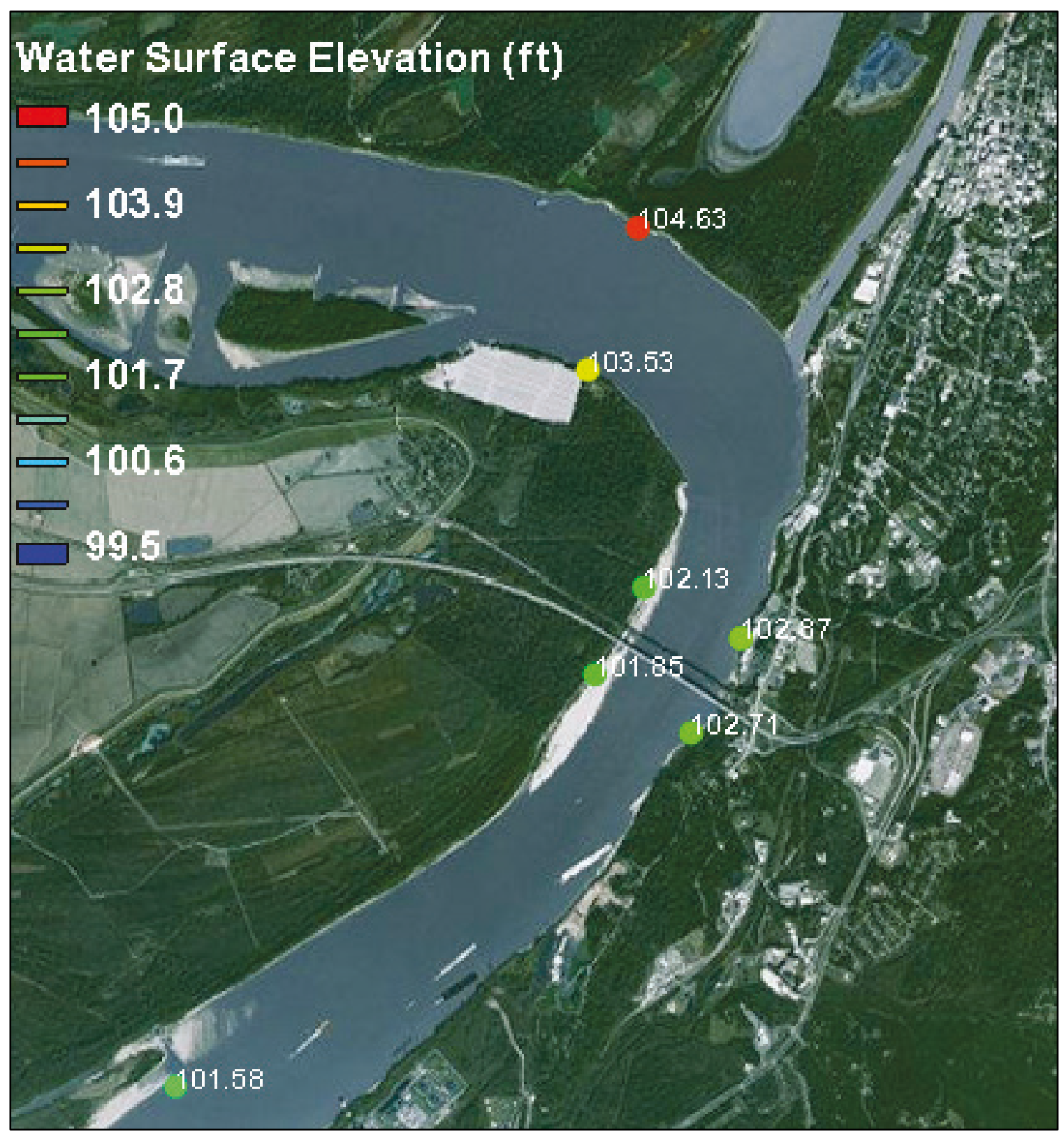


Table 4. Water surface elevations at Vicksburg, MS.

\begin{tabular}{|c|c|c|c|}
\hline \multirow[b]{2}{*}{ Date/Time } & \multicolumn{2}{|c|}{$\begin{array}{c}\text { State Plane } \\
\text { Coordinate System } \\
\text { MS WEST } 2302 \\
\text { Survey (feet) }\end{array}$} & \multirow{2}{*}{$\begin{array}{l}\text { NAVD } 88 \\
\text { (feet) } \\
\text { Elevation }\end{array}$} \\
\hline & Northing & Easting & \\
\hline 5/12/2011 17:36 & 1032599 & 2122979 & 101.43 \\
\hline 5/12/2011 17:49 & 1039280 & 2126686 & 101.82 \\
\hline $5 / 13 / 201111: 43$ & 1034386 & 2119542 & 102.08 \\
\hline $5 / 13 / 201111: 49$ & 1031538 & 2117933 & 101.24 \\
\hline $5 / 13 / 201111: 58$ & 1026332 & 2119273 & 100.06 \\
\hline $5 / 13 / 201112: 03$ & 1025681 & 2121473 & 101.24 \\
\hline $5 / 13 / 201112: 10$ & 1022720 & 2120226 & 100.51 \\
\hline 5/13/2011 12:15 & 1024248 & 2117962 & 99.68 \\
\hline 5/16/2011 16:28 & 1014109 & 2107642 & 101.58 \\
\hline 5/16/2011 16:48 & 1022617 & 2120063 & 102.71 \\
\hline 5/16/2011 16:51 & 1024021 & 2117747 & 101.85 \\
\hline 5/16/2011 16:54 & 1026118 & 2118927 & 102.13 \\
\hline 5/16/2011 16:59 & 1024895 & 2121264 & 102.87 \\
\hline 5/16/2011 17:05 & 1034768 & 2118784 & 104.63 \\
\hline 5/16/2011 17:07 & 1031351 & 2117576 & 103.53 \\
\hline
\end{tabular}

Table 5. Super elevations and setup.

\begin{tabular}{|c|c|c|c|c|c|c|c|}
\hline & \multirow{2}{*}{ Location } & \multicolumn{4}{|c|}{13 May 2011 } & \multicolumn{3}{c|}{16 May 2011 } \\
\cline { 3 - 8 } & Mile & Right Bank & Left bank & Setup & Right Bank & Left bank & Setup \\
\hline In bend & 438 & 101.24 & 102.08 & 0.84 & 103.53 & 104.63 & 1.1 \\
\hline $\begin{array}{c}\text { Upstream } \\
\text { bridge }\end{array}$ & 436 & 100.60 & 101.24 & 0.64 & 102.13 & 102.87 & 0.74 \\
\hline $\begin{array}{c}\text { Downstream } \\
\text { bridge }\end{array}$ & 436.5 & 99.68 & 100.51 & 0.83 & 101.85 & 102.71 & 0.86 \\
\hline
\end{tabular}




\section{Conclusions}

Processing of the 2011 bathymetry data proved very useful. These new data allowed bed-load calculations to populate the rating curves at extreme high-flow events. The data continued to follow the same trend observed in prior data with bedload continuing to increase significantly as flow increases. Both the Vicksburg and the Lower Mississippi River (LMR) rating curve are now bounded by two extreme events, the 2011 Flood and the 2012 drought. These rating curves can be a powerful tool to look at past sediment delivery and predict future sediment delivery given a future forecasted hydrograph.

The Vicksburg data matches well with the LMR data and is a good representation of the LMR. Future measurements at this site would be beneficial to continue to populate the rating curves and build a larger database of bed-load observations. This database could be used for further investigations such as determining other factors besides flow that influence bed-load transport. Continuous measurements over a year would produce a significant amount of data and would also allow the ability to see the evolution of dunes and how they grow and shrink during a hydrograph. This can somewhat be seen in the 2011 data in Appendix A. As flow rates decrease, the large dunes began to decrease in size. This morphing appears to be driven by smaller dunes translating over the tops of the larger dunes as can be seen in the 03 June 2011 survey and profiles in Appendix A.

Continuous improvements of the method, such as fewer data requirements, along with the close proximity of the Vicksburg site to the U.S. Army Engineer Research and Development Center, could make continuous bed-load measurements more economically feasible. The main variables affecting this would be the total length of time (e.g., 1 year) and the interval (e.g., weekly).

The water surface measurements also provide a significant amount of data. The water surface profile provides a continuous dataset along the Mississippi River between Rosedale, MS, and Natchez, MS, that is also consistent with the gage observations during the 2011 Flood. This provides high spatial fidelity water surface elevations during an extreme event. The water surface measurements at the Yazoo Backwater levee captured a unique phenomenon as a drawdown occurred at the levee intersection with the mainline levee. This data can be very useful in better 
understanding and predicting the localized energy loss and levee overtopping potential at this location. The super elevation measurements near the Vicksburg bridge captured a 1-foot higher water surface elevation on the left bank (descending) than the right bank (descending) in the bend of the river during an extreme flood. 


\section{References}

Abraham D., R. Kuhnle, and A. J. Odgaard. 2011. "Validation of Bed-Load Transport Measurements with Time-Sequenced Bathymetric Data." ASCE Journal of Hydraulic Engineering 137(7): 723-728. 


\section{Appendix A: Dune Survey and Profile Plots}

This appendix consists of an example dune survey and an example dune translation profile for each survey date. The plan views are a combination of seven surveyed swaths that are numbered 1 through 7 from right descending bank to the left. The profiles represent a longitudinal section. The numbers in the legend of the profiles represent the swath number and the time of the survey.

Figure 20. 15 May 2011 dune field.

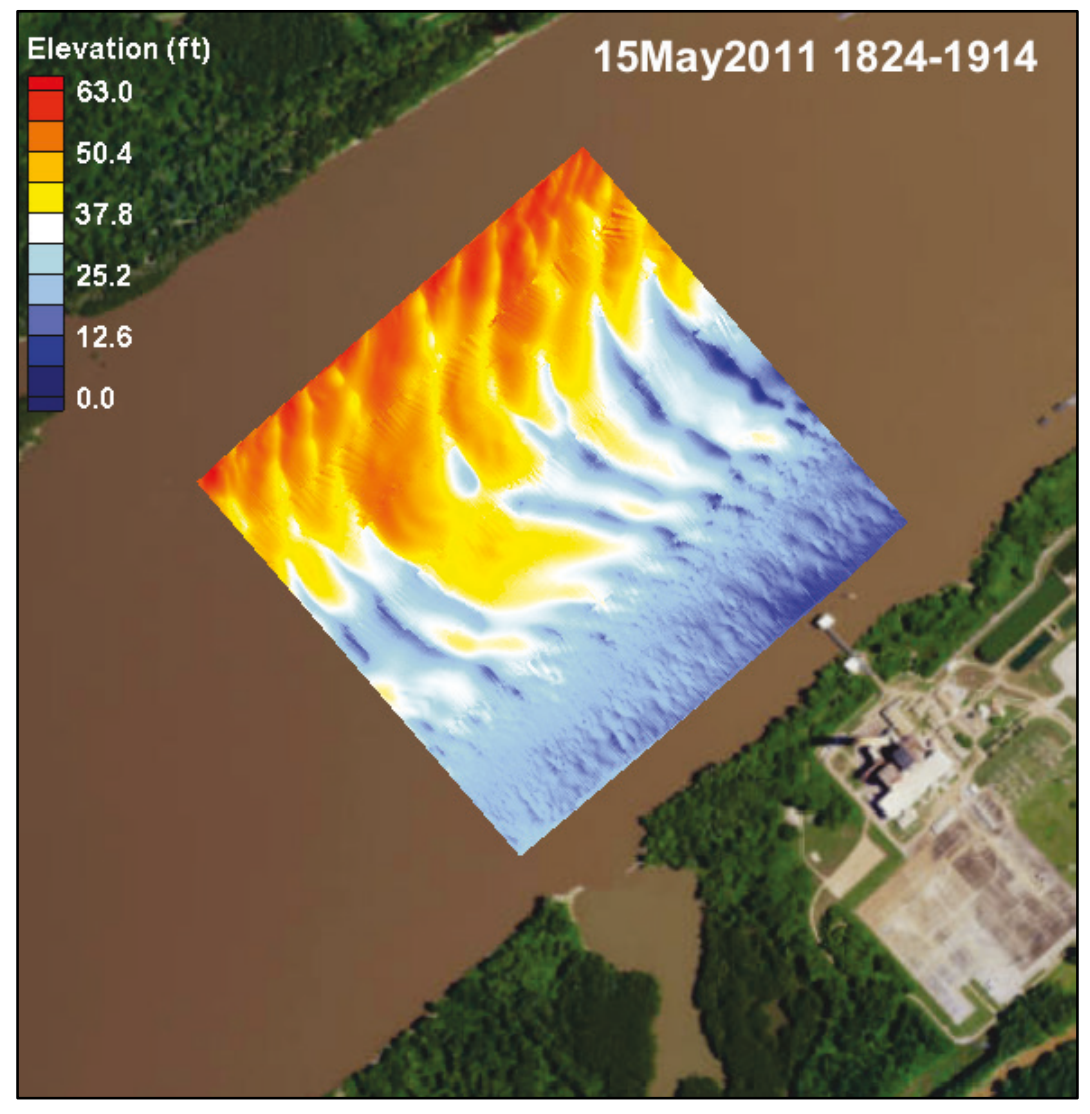


Figure 21. 15 May 2011 example profile.

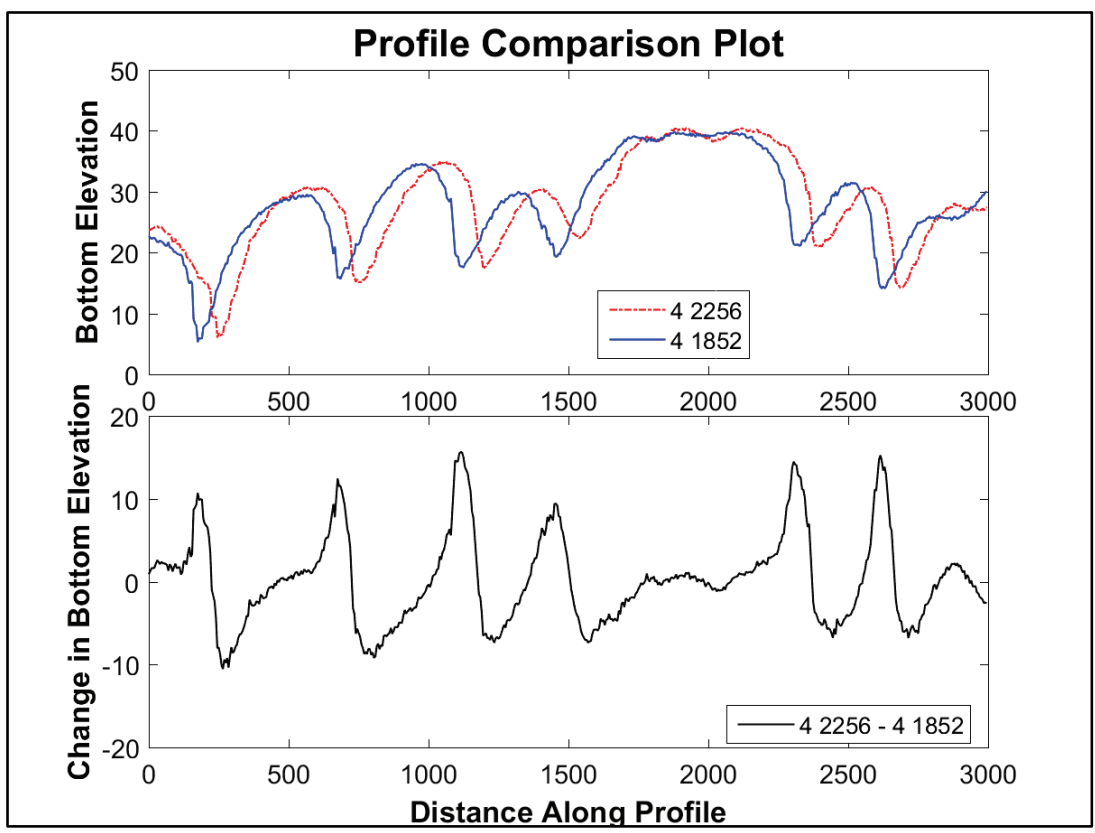

Figure 22. 19 May 2011 dune field.

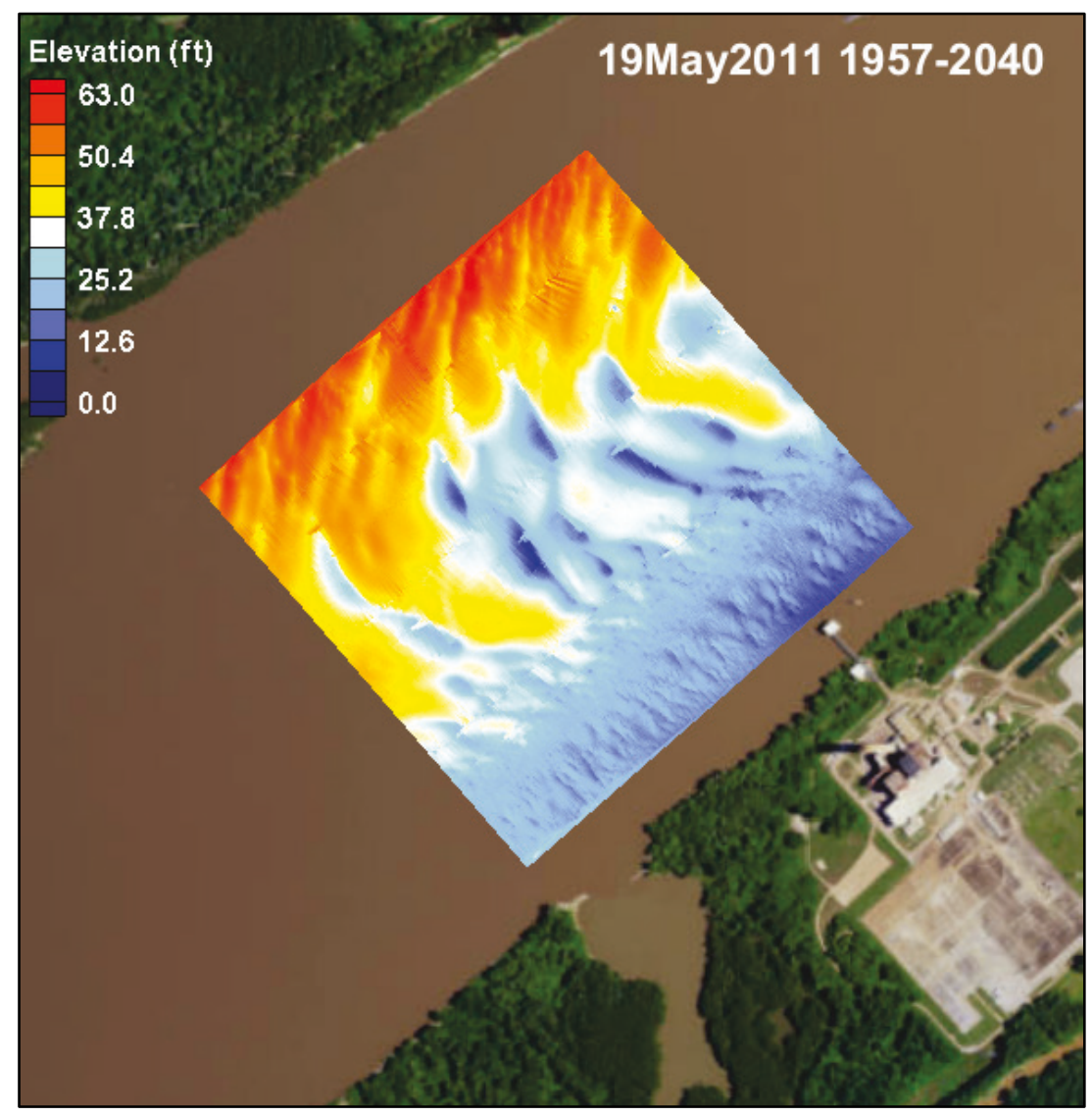


Figure 23. 19 May 2011 example profile.

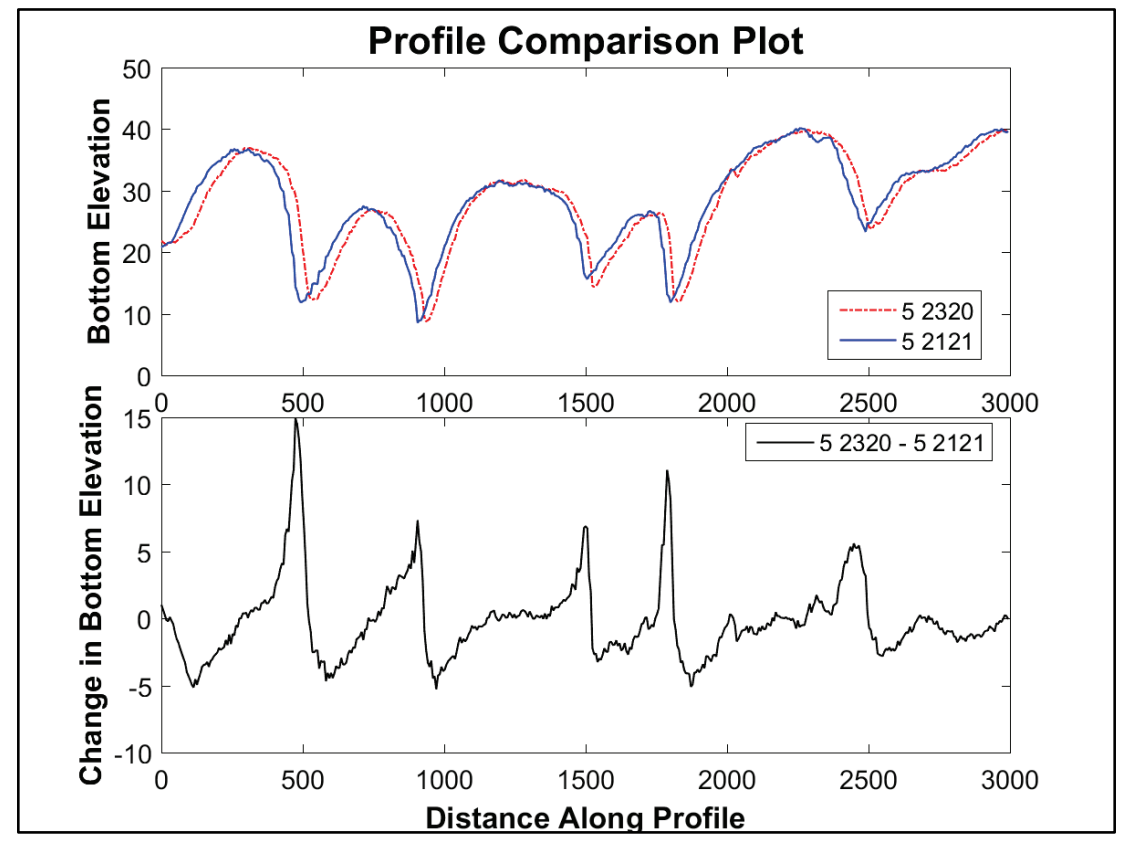

Figure 24. 26 May 2011 dune field.

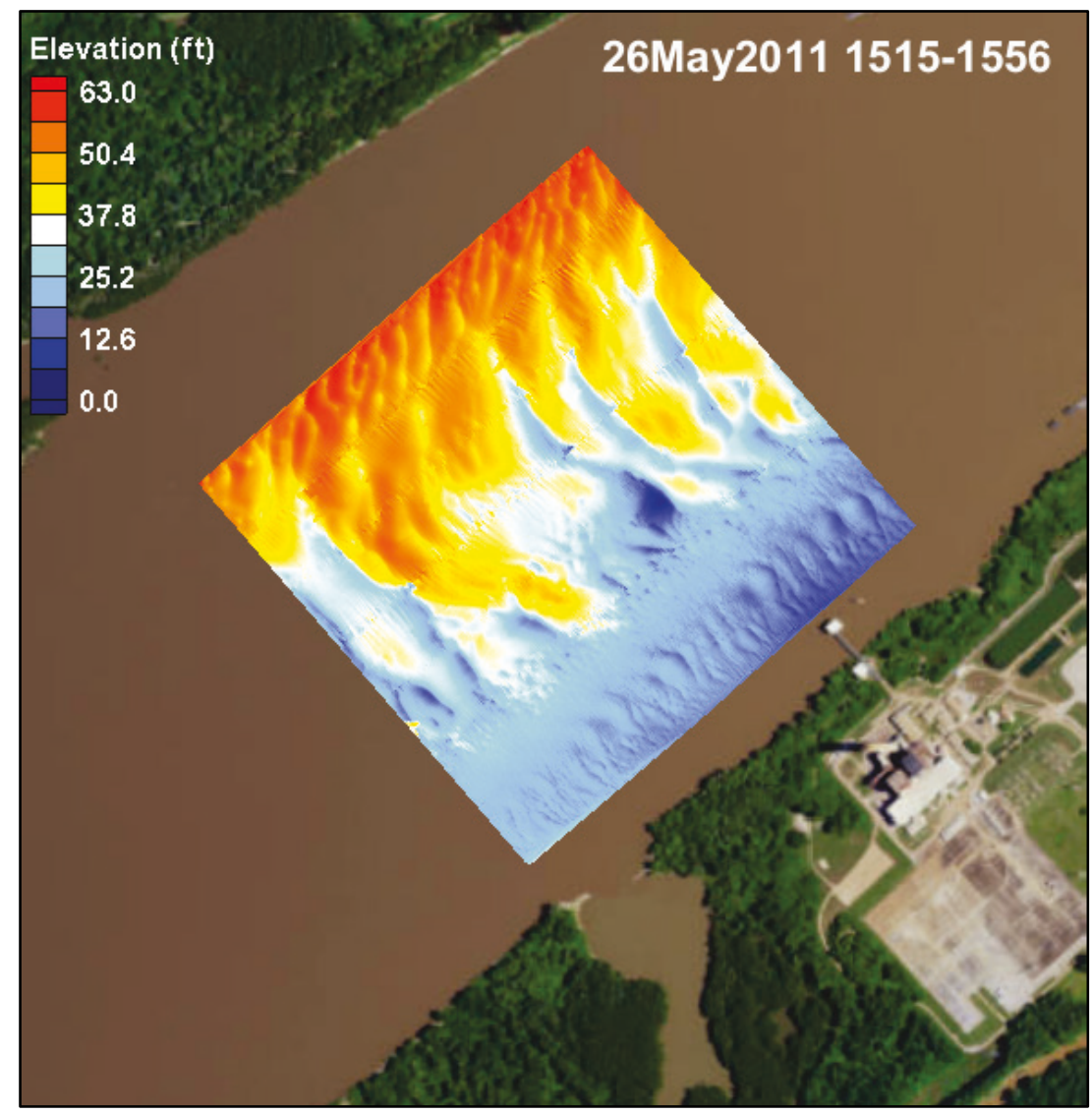


Figure 25. 26 May 2011 example profile.

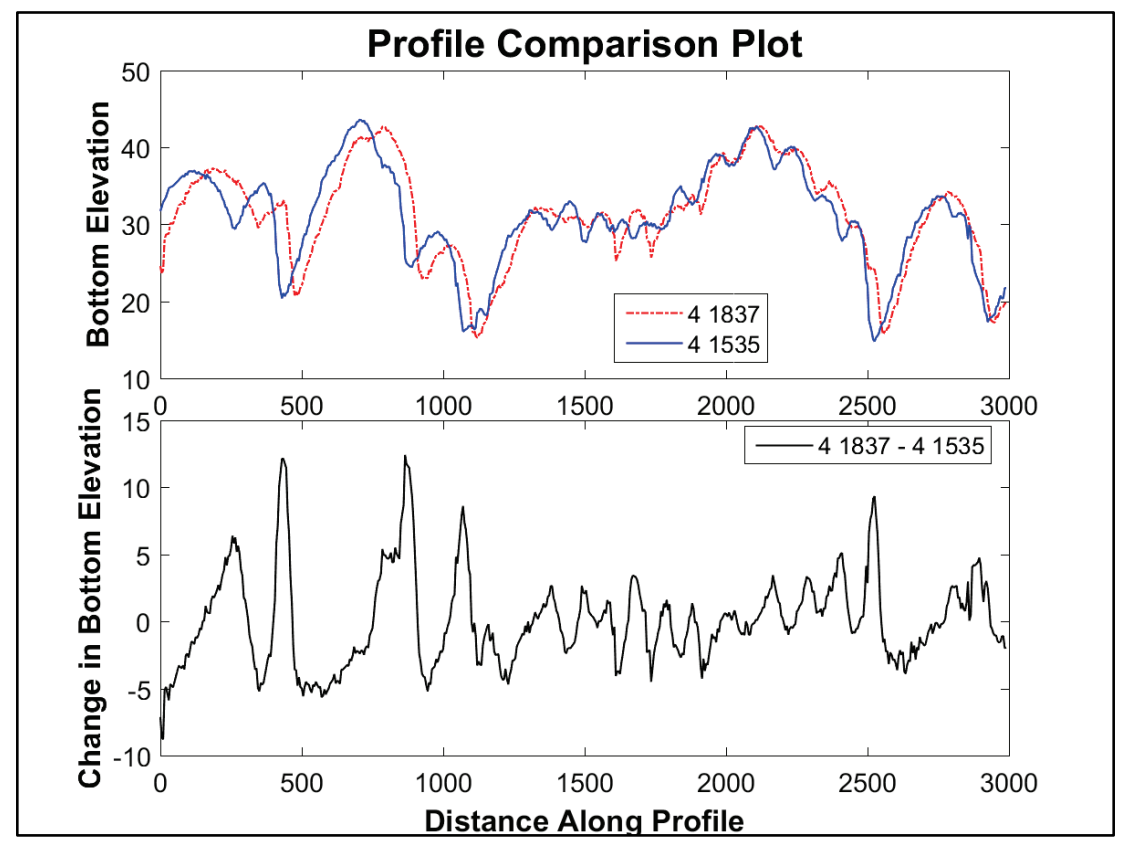

Figure 26. 31 May 2011 dune field.

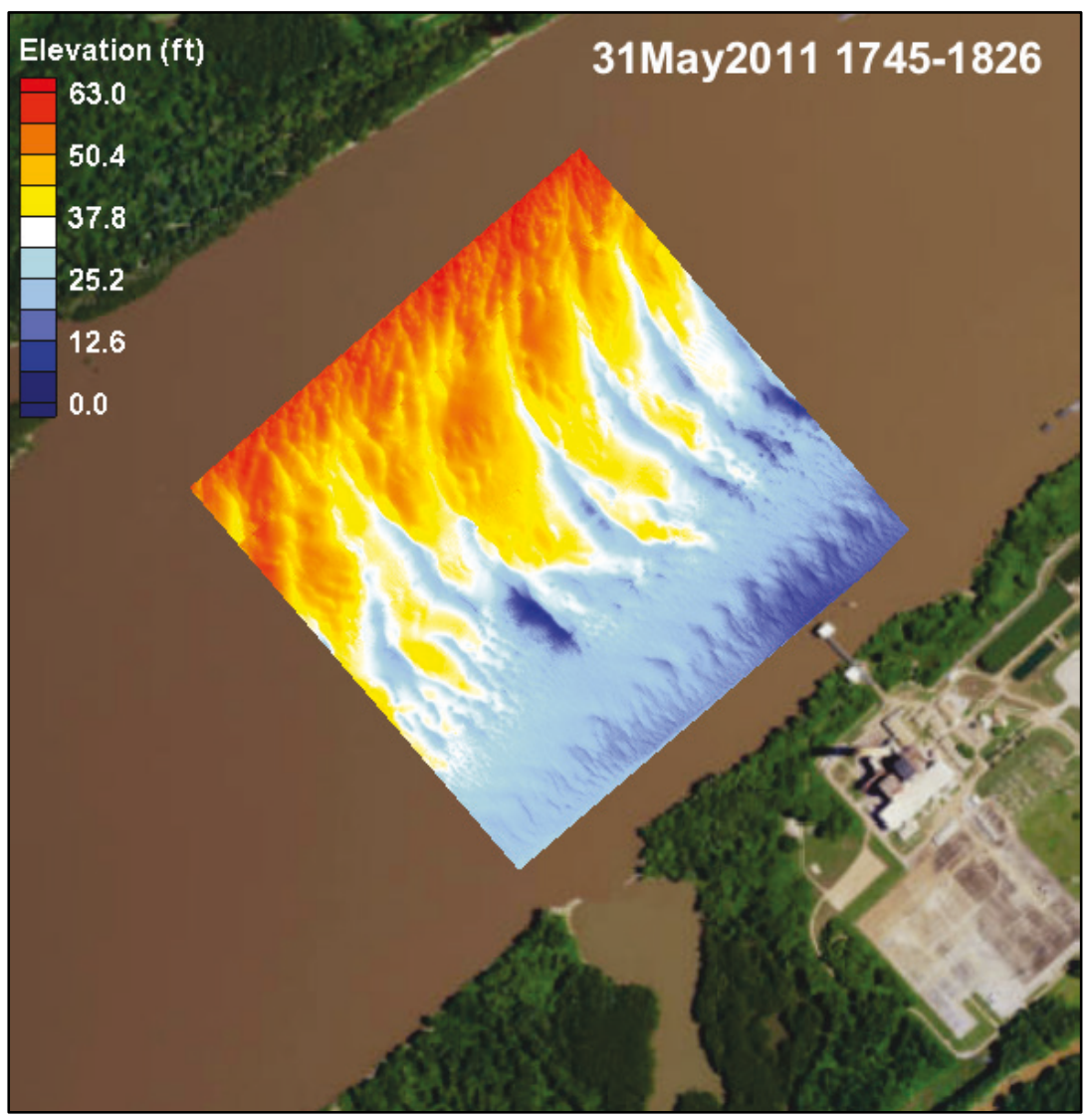


Figure 27. 31 May 2011 example profile.

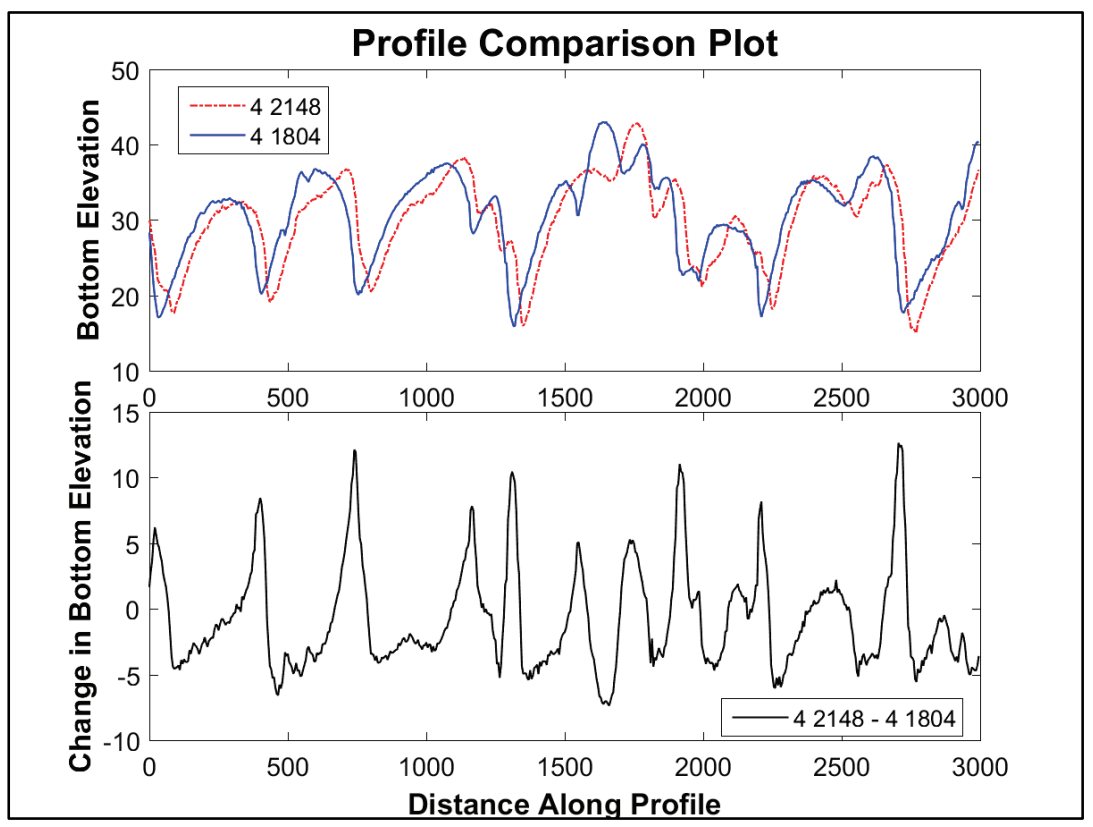

Figure 28. 03 June 2011 dune field.

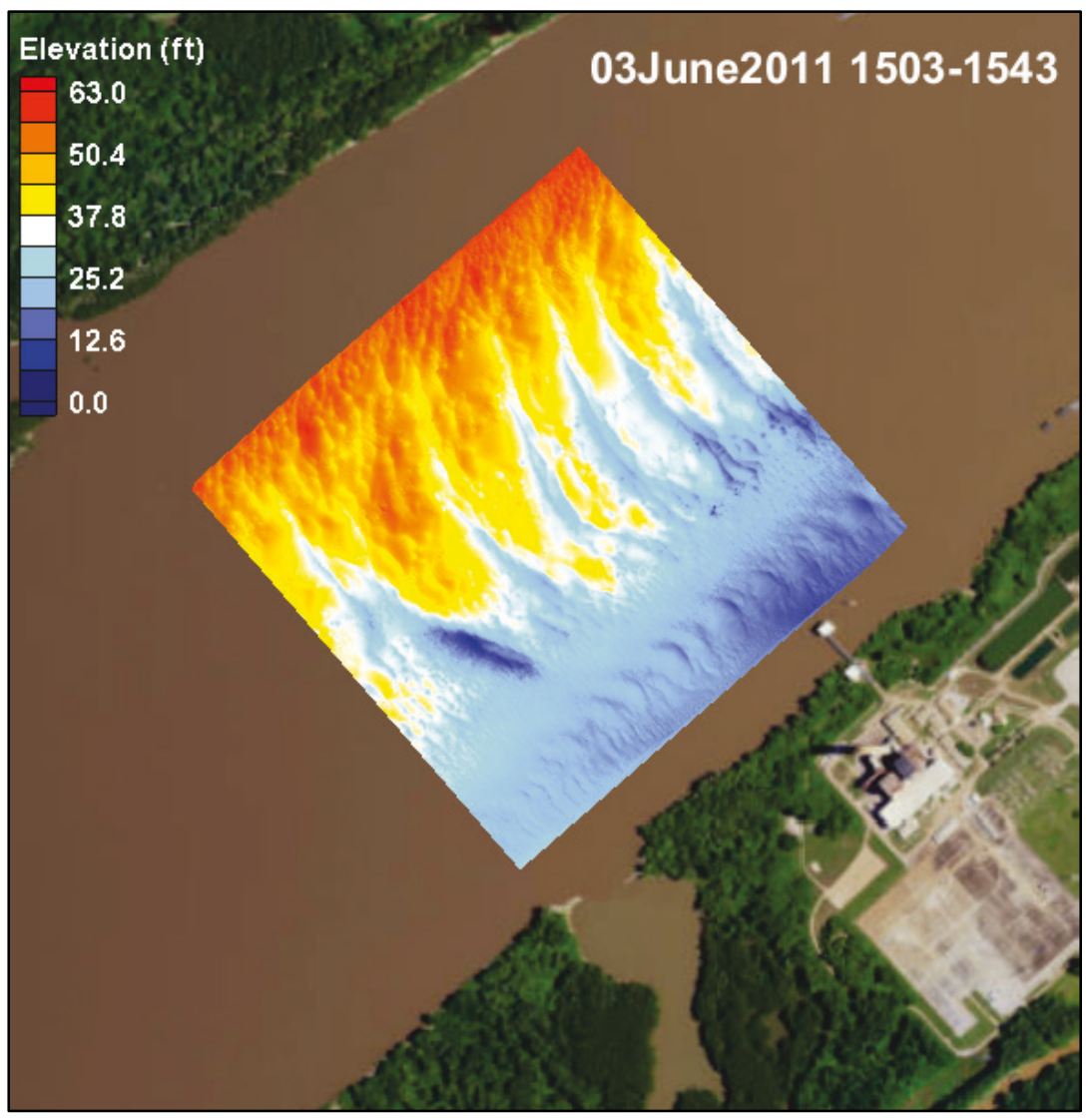


Figure 29. 03 June 2011 example profile.

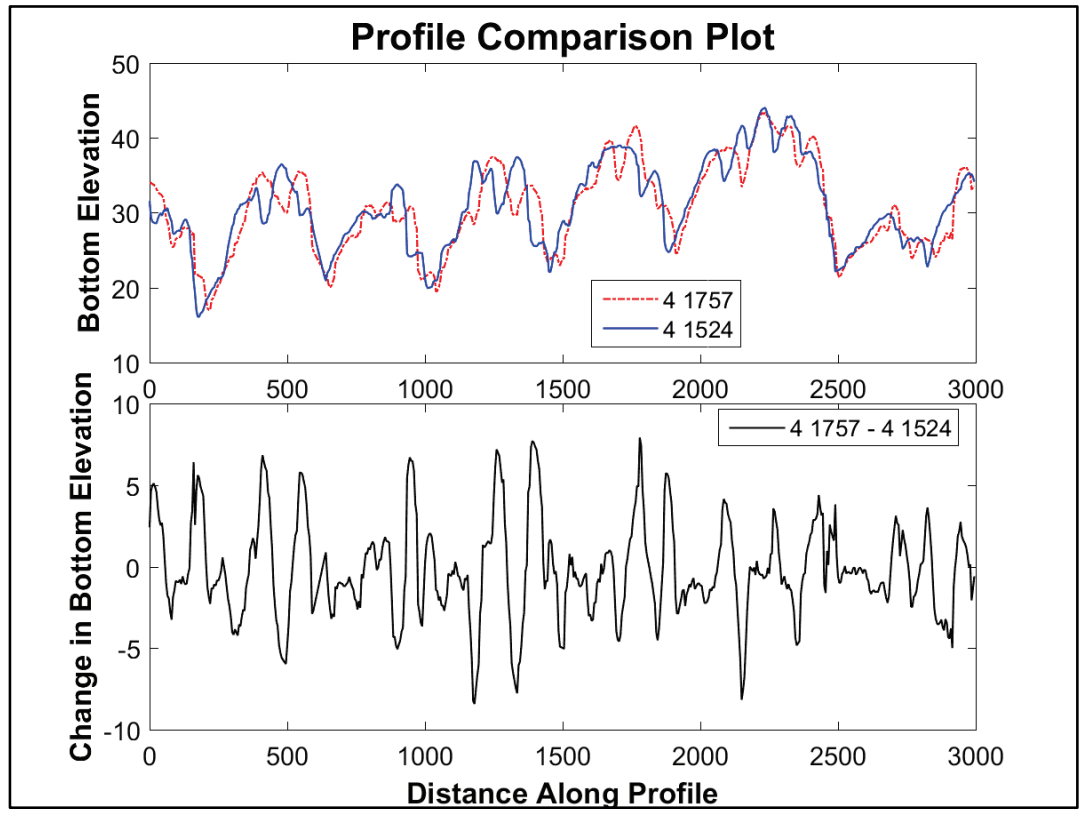




\section{Appendix B: Water Surface Profile Data}

This appendix includes tabulated information for the water surface profile from river mile 597 to 362 .

\begin{tabular}{|c|c|c|c|c|c|c|c|c|}
\hline River Mile & $\begin{array}{l}\text { GPS Time } \\
\text { (seconds) }\end{array}$ & $\begin{array}{c}\text { Easting SP } \\
\text { (feet) }\end{array}$ & $\begin{array}{l}\text { Northing SP } \\
\text { (feet) }\end{array}$ & $\begin{array}{l}\text { Elevation } \\
\text { Geoid } 03\end{array}$ & $\begin{array}{l}\text { Standard } \\
\text { Deviation }\end{array}$ & Latitude & Longitude & $\begin{array}{l}\text { Ellipsoid Ht } \\
\text { U.S. Survey } \\
\text { (feet) }\end{array}$ \\
\hline 362 & 343754 & 1952484.99 & 747626.678 & 78.248 & 0.056 & 31.550843 & -91.437943 & -6.526 \\
\hline 362.5 & 343390 & 1954853.22 & 749311.734 & 78.461 & 0.056 & 31.555540 & -91.430396 & -6.503 \\
\hline 363 & 343214 & 1956717.59 & 750574.389 & 78.622 & 0.056 & 31.559062 & -91.424452 & -6.297 \\
\hline 363.5 & 343046 & 1958843.23 & 751930.217 & 78.580 & 0.062 & 31.562847 & -91.417672 & -6.291 \\
\hline 364 & 342910 & 1960560.34 & 753402.469 & 79.275 & 0.062 & 31.566940 & -91.412207 & -5.427 \\
\hline 364.5 & 342847 & 1961961.15 & 755595.401 & 79.595 & 0.075 & 31.573005 & -91.407779 & -3.859 \\
\hline 365 & 342806 & 1962508.69 & 757963.112 & 79.457 & 0.072 & 31.579528 & -91.406096 & -4.473 \\
\hline 365.5 & 342765 & 1963041.89 & 760478.812 & 79.571 & 0.075 & 31.586457 & -91.404463 & -4.202 \\
\hline 366 & 342723 & 1963259.13 & 763057.611 & 79.673 & 0.075 & 31.593551 & -91.403846 & -3.917 \\
\hline 366.5 & 342680 & 1963518.33 & 765612.64 & 80.182 & 0.072 & 31.600581 & -91.403094 & -3.395 \\
\hline 367 & 342636 & 1963666.43 & 768196.789 & 80.479 & 0.072 & 31.607687 & -91.402700 & -3.225 \\
\hline 367.5 & 342594 & 1963931.00 & 770704.854 & 80.762 & 0.075 & 31.614588 & -91.401929 & -2.971 \\
\hline 368 & 342554 & 1964344.09 & 773132.783 & 80.831 & 0.075 & 31.621273 & -91.400678 & -3.031 \\
\hline 368.5 & 342511 & 1964719.60 & 775815.373 & 81.037 & 0.072 & 31.628656 & -91.399556 & -2.618 \\
\hline 369 & 342467 & 1964935.33 & 778492.211 & 81.115 & 0.075 & 31.636019 & -91.398947 & -2.518 \\
\hline 369.5 & 342411 & 1964686.02 & 781318.369 & 80.287 & 0.066 & 31.643780 & -91.399837 & -3.352 \\
\hline 370 & 342355 & 1964412.81 & 784244.251 & 81.028 & 0.079 & 31.651815 & -91.400806 & -2.524 \\
\hline 370.5 & 342309 & 1964474.56 & 787102.292 & 80.815 & 0.075 & 31.659672 & -91.400698 & -2.998 \\
\hline 371 & 342264 & 1964569.56 & 789873.948 & 81.511 & 0.072 & 31.667293 & -91.400479 & -2.140 \\
\hline 371.5 & 342225 & 1964723.48 & 792272.99 & 81.651 & 0.075 & 31.673891 & -91.400060 & -1.934 \\
\hline 372 & 342186 & 1965079.30 & 794614.377 & 82.003 & 0.075 & 31.680336 & -91.398990 & -1.740 \\
\hline 372.5 & 342138 & 1965259.81 & 797423.724 & 82.534 & 0.072 & 31.688062 & -91.398498 & -0.832 \\
\hline 373 & 342093 & 1965559.96 & 799986.588 & 82.916 & 0.072 & 31.695115 & -91.397614 & -0.763 \\
\hline 373.5 & 342048 & 1966000.92 & 802273.389 & 82.814 & 0.072 & 31.701412 & -91.396268 & -0.708 \\
\hline 374 & 341991 & 1966629.22 & 804652.263 & 82.841 & 0.066 & 31.707967 & -91.394322 & -0.820 \\
\hline 374.5 & 341939 & 1967423.23 & 806877.901 & 83.635 & 0.072 & 31.714105 & -91.391839 & -0.106 \\
\hline 375 & 341884 & 1968611.44 & 809085.06 & 83.465 & 0.072 & 31.720204 & -91.388087 & 0.108 \\
\hline 375.5 & 341839 & 1969992.27 & 811224.443 & 83.871 & 0.072 & 31.726120 & -91.383714 & 0.135 \\
\hline
\end{tabular}




\begin{tabular}{|c|c|c|c|c|c|c|c|c|}
\hline River Mile & $\begin{array}{l}\text { GPS Time } \\
\text { (seconds) }\end{array}$ & $\begin{array}{l}\text { Easting SP } \\
\text { (feet) }\end{array}$ & $\begin{array}{l}\text { Northing SP } \\
\quad \text { (feet) }\end{array}$ & $\begin{array}{l}\text { Elevation } \\
\text { Geoid } 03\end{array}$ & $\begin{array}{l}\text { Standard } \\
\text { Deviation }\end{array}$ & Latitude & Longitude & $\begin{array}{l}\text { Ellipsoid Ht } \\
\text { U.S. Survey } \\
\quad \text { (feet) }\end{array}$ \\
\hline 376 & 341753 & 1971462.05 & 813481.481 & 83.965 & 0.069 & 31.732363 & -91.379057 & 0.692 \\
\hline 376.5 & 341705 & 1972688.72 & 816004.499 & 84.168 & 0.072 & 31.739330 & -91.375189 & 0.721 \\
\hline 377 & 341658 & 1973893.76 & 818501.869 & 84.495 & 0.072 & 31.746226 & -91.371390 & 1.053 \\
\hline 377.5 & 341611 & 1974872.83 & 821065.714 & 84.687 & 0.079 & 31.753298 & -91.368320 & 1.198 \\
\hline 378 & 341564 & 1975770.43 & 823616.689 & 84.807 & 0.082 & 31.760333 & -91.365510 & 1.259 \\
\hline 378.5 & 341439 & 1976519.76 & 826354.918 & 85.096 & 0.085 & 31.767879 & -91.363183 & 1.539 \\
\hline 379 & 341398 & 1976656.59 & 828750.026 & 85.195 & 0.098 & 31.774465 & -91.362816 & 1.742 \\
\hline 379.5 & 341354 & 1976986.28 & 831314.385 & 85.260 & 0.102 & 31.781522 & -91.361833 & 1.678 \\
\hline 380 & 341309 & 1977436.29 & 833923.754 & 85.503 & 0.105 & 31.788706 & -91.360464 & 2.016 \\
\hline 380.5 & 341264 & 1977841.90 & 836526.628 & 85.481 & 0.105 & 31.795870 & -91.359238 & 1.986 \\
\hline 381 & 341219 & 1978389.82 & 839074.409 & 85.648 & 0.112 & 31.802887 & -91.357552 & 2.153 \\
\hline 381.5 & 341171 & 1978939.68 & 841822.212 & 85.814 & 0.112 & 31.810454 & -91.355865 & 2.314 \\
\hline 382 & 341124 & 1979627.39 & 844494.921 & 86.058 & 0.115 & 31.817818 & -91.353733 & 2.524 \\
\hline 382.5 & 341079 & 1980297.75 & 847046.051 & 86.295 & 0.115 & 31.824847 & -91.351652 & 2.873 \\
\hline 383 & 341035 & 1980960.81 & 849548.496 & 86.425 & 0.115 & 31.831742 & -91.349593 & 2.914 \\
\hline 383.5 & 340988 & 1981575.26 & 852165.827 & 87.082 & 0.121 & 31.838952 & -91.347693 & 3.611 \\
\hline 384 & 340940 & 1982955.18 & 854428.859 & 87.308 & 0.125 & 31.845207 & -91.343318 & 3.803 \\
\hline 384.5 & 340896 & 1985144.21 & 855603.842 & 87.397 & 0.128 & 31.848492 & -91.336305 & 3.907 \\
\hline 385 & 340851 & 1987407.28 & 856809.459 & 87.474 & 0.128 & 31.851863 & -91.329053 & 3.957 \\
\hline 385.5 & 340809 & 1989618.51 & 857716.563 & 87.580 & 0.128 & 31.854412 & -91.321959 & 4.043 \\
\hline 386 & 340765 & 1992132.75 & 858069.744 & 87.501 & 0.135 & 31.855445 & -91.313873 & 3.962 \\
\hline 386.5 & 340721 & 1994704.31 & 858021.647 & 87.599 & 0.138 & 31.855377 & -91.305590 & 4.110 \\
\hline 387 & 340676 & 1997303.71 & 858143.436 & 87.675 & 0.131 & 31.855775 & -91.297223 & 4.174 \\
\hline 387.5 & 340631 & 1999872.71 & 858320.98 & 87.790 & 0.131 & 31.856326 & -91.288955 & 4.261 \\
\hline 388 & 340584 & 2002481.65 & 858652.535 & 87.947 & 0.128 & 31.857300 & -91.280563 & 4.364 \\
\hline 388.5 & 340538 & 2005022.20 & 858929.539 & 87.969 & 0.128 & 31.858122 & -91.272389 & 4.415 \\
\hline 389 & 340492 & 2007557.59 & 859440.554 & 88.020 & 0.125 & 31.859586 & -91.264238 & 4.414 \\
\hline 389.5 & 340444 & 2010181.25 & 860111.376 & 88.218 & 0.121 & 31.861492 & -91.255807 & 4.616 \\
\hline 390 & 340391 & 2012922.97 & 861529.834 & 88.446 & 0.121 & 31.865454 & -91.247016 & 4.908 \\
\hline 390.5 & 340348 & 2015154.12 & 862817.755 & 88.292 & 0.118 & 31.869046 & -91.239864 & 4.666 \\
\hline 391 & 340303 & 2017458.60 & 864280.568 & 88.452 & 0.115 & 31.873119 & -91.232481 & 4.946 \\
\hline 391.5 & 340258 & 2019591.40 & 865943.943 & 88.684 & 0.108 & 31.877739 & -91.225655 & 5.001 \\
\hline 392 & 340213 & 2021741.36 & 867581.362 & 88.990 & 0.108 & 31.882289 & -91.218773 & 5.354 \\
\hline
\end{tabular}




\begin{tabular}{|c|c|c|c|c|c|c|c|c|}
\hline River Mile & $\begin{array}{l}\text { GPS Time } \\
\text { (seconds) }\end{array}$ & $\begin{array}{l}\text { Easting SP } \\
\quad \text { (feet) }\end{array}$ & $\begin{array}{l}\text { Northing SP } \\
\quad \text { (feet) }\end{array}$ & $\begin{array}{l}\text { Elevation } \\
\text { Geoid } 03\end{array}$ & $\begin{array}{l}\text { Standard } \\
\text { Deviation }\end{array}$ & Latitude & Longitude & $\begin{array}{l}\text { Ellipsoid Ht } \\
\text { U.S. Survey } \\
\quad \text { (feet) }\end{array}$ \\
\hline 392.5 & 340167 & 2023458.14 & 869701.058 & 89.533 & 0.105 & 31.888153 & -91.213298 & 5.801 \\
\hline 393 & 340123 & 2024926.32 & 871861.607 & 89.718 & 0.102 & 31.894124 & -91.208625 & 5.932 \\
\hline 393.5 & 340079 & 2026041.15 & 874118.39 & 89.665 & 0.098 & 31.900352 & -91.205091 & 5.948 \\
\hline 394 & 340037 & 2027020.13 & 876400.682 & 89.670 & 0.092 & 31.906647 & -91.201996 & 5.975 \\
\hline 394.5 & 339994 & 2027849.88 & 878837.819 & 89.491 & 0.092 & 31.913364 & -91.199385 & 5.833 \\
\hline 395 & 339949 & 2028417.88 & 881383.028 & 89.930 & 0.089 & 31.920372 & -91.197620 & 6.226 \\
\hline 395.5 & 339902 & 2029059.31 & 883996.743 & 89.714 & 0.089 & 31.927570 & -91.195620 & 6.048 \\
\hline 396 & 339852 & 2029193.26 & 886963.896 & 89.862 & 0.085 & 31.935729 & -91.195265 & 6.375 \\
\hline 396.5 & 339808 & 2029848.62 & 889510.813 & 89.724 & 0.085 & 31.942743 & -91.193218 & 5.976 \\
\hline 397 & 339766 & 2030505.91 & 891949.429 & 90.348 & 0.089 & 31.949461 & -91.191161 & 6.571 \\
\hline 397.5 & 339724 & 2031332.20 & 894329.509 & 91.031 & 0.085 & 31.956020 & -91.188558 & 7.244 \\
\hline 398 & 339681 & 2032357.79 & 896604.141 & 91.332 & 0.085 & 31.962295 & -91.185309 & 7.530 \\
\hline 398.5 & 339641 & 2033662.42 & 898522.968 & 91.446 & 0.085 & 31.967597 & -91.181152 & 7.860 \\
\hline 399 & 339603 & 2035248.27 & 900128.525 & 91.619 & 0.082 & 31.972044 & -91.176079 & 8.030 \\
\hline 399.5 & 339558 & 2037462.34 & 901570.827 & 91.489 & 0.085 & 31.976056 & -91.168975 & 7.716 \\
\hline 400 & 339519 & 2039394.71 & 902782.1 & 92.090 & 0.082 & 31.979426 & -91.162774 & 8.200 \\
\hline 400.5 & 339485 & 2041320.03 & 903386.005 & 91.889 & 0.085 & 31.981126 & -91.156580 & 7.921 \\
\hline 401 & 339446 & 2043614.61 & 903837.319 & 92.326 & 0.085 & 31.982414 & -91.149192 & 8.578 \\
\hline 401.5 & 339395 & 2046606.40 & 904175.915 & 92.518 & 0.089 & 31.983407 & -91.139552 & 8.831 \\
\hline 402 & 339347 & 2049417.47 & 904431.876 & 92.508 & 0.085 & 31.984168 & -91.130492 & 8.622 \\
\hline 402.5 & 339300 & 2052018.92 & 905092.826 & 92.577 & 0.079 & 31.986037 & -91.122118 & 8.837 \\
\hline 403 & 339262 & 2054211.12 & 905316.636 & 92.815 & 0.082 & 31.986696 & -91.115054 & 9.161 \\
\hline 403.5 & 339217 & 2056784.08 & 905868.228 & 92.986 & 0.079 & 31.988263 & -91.106769 & 9.344 \\
\hline 404 & 339169 & 2059392.11 & 906916.333 & 93.055 & 0.075 & 31.991194 & -91.098381 & 9.277 \\
\hline 404.5 & 339122 & 2061501.02 & 908688.753 & 93.190 & 0.079 & 31.996107 & -91.091620 & 9.406 \\
\hline 405 & 339081 & 2063234.00 & 910361.128 & 93.120 & 0.075 & 32.000737 & -91.086068 & 9.241 \\
\hline 405.5 & 339035 & 2065202.20 & 912237.37 & 93.459 & 0.079 & 32.005932 & -91.079761 & 9.833 \\
\hline 406 & 338988 & 2066729.84 & 914546.425 & 93.749 & 0.075 & 32.012307 & -91.074884 & 9.951 \\
\hline 406.5 & 338943 & 2067859.36 & 916940.155 & 93.881 & 0.075 & 32.018908 & -91.071293 & 10.006 \\
\hline 407 & 338898 & 2068934.03 & 919302.053 & 94.152 & 0.075 & 32.025420 & -91.067878 & 10.220 \\
\hline 407.5 & 338852 & 2070108.33 & 921704.916 & 94.556 & 0.075 & 32.032047 & -91.064142 & 10.704 \\
\hline 408 & 338802 & 2071163.83 & 924385.138 & 94.817 & 0.075 & 32.039434 & -91.060794 & 10.917 \\
\hline 408.5 & 338751 & 2071994.32 & 927139.23 & 95.085 & 0.075 & 32.047019 & -91.058174 & 11.146 \\
\hline
\end{tabular}




\begin{tabular}{|c|c|c|c|c|c|c|c|c|}
\hline River Mile & $\begin{array}{l}\text { GPS Time } \\
\text { (seconds) }\end{array}$ & $\begin{array}{l}\text { Easting SP } \\
\text { (feet) }\end{array}$ & $\begin{array}{l}\text { Northing SP } \\
\quad \text { (feet) }\end{array}$ & $\begin{array}{l}\text { Elevation } \\
\text { Geoid } 03\end{array}$ & $\begin{array}{l}\text { Standard } \\
\text { Deviation }\end{array}$ & Latitude & Longitude & $\begin{array}{l}\text { Ellipsoid Ht } \\
\text { U.S. Survey } \\
\quad \text { (feet) }\end{array}$ \\
\hline 409 & 338693 & 2072079.19 & 930352.59 & 95.502 & 0.072 & 32.055853 & -91.057970 & 11.532 \\
\hline 409.5 & 338643 & 2071205.98 & 932934.64 & 95.639 & 0.072 & 32.062934 & -91.060844 & 11.646 \\
\hline 410 & 338599 & 2070255.88 & 935077.403 & 95.762 & 0.072 & 32.068806 & -91.063958 & 11.750 \\
\hline 410.5 & 338553 & 2068800.20 & 936995.285 & 95.924 & 0.072 & 32.074050 & -91.068699 & 11.900 \\
\hline 411 & 338503 & 2066827.07 & 938747.517 & 95.988 & 0.072 & 32.078829 & -91.075107 & 11.941 \\
\hline 411.5 & 338454 & 2064761.08 & 940495.355 & 96.003 & 0.069 & 32.083594 & -91.081816 & 11.936 \\
\hline 412 & 338408 & 2062990.65 & 942405.545 & 96.051 & 0.069 & 32.088811 & -91.087575 & 12.016 \\
\hline 412.5 & 338357 & 2061215.09 & 944732.426 & 96.142 & 0.069 & 32.095172 & -91.093360 & 12.136 \\
\hline 413 & 338311 & 2059795.78 & 946949.42 & 96.340 & 0.069 & 32.101238 & -91.097994 & 12.230 \\
\hline 413.5 & 338268 & 2058457.95 & 948981.623 & 96.747 & 0.069 & 32.106798 & -91.102360 & 12.628 \\
\hline 414 & 338228 & 2057205.00 & 950914.388 & 97.081 & 0.072 & 32.112086 & -91.106451 & 12.920 \\
\hline 414.5 & 338106 & 2058200.77 & 956449.449 & 97.693 & 0.072 & 32.127319 & -91.103363 & 13.506 \\
\hline 415 & 338085 & 2059329.08 & 956799.59 & 97.697 & 0.072 & 32.128303 & -91.099727 & 13.500 \\
\hline 415.5 & 338050 & 2061119.11 & 957548.591 & 97.875 & 0.069 & 32.130397 & -91.093962 & 13.682 \\
\hline 416 & 337991 & 2064193.93 & 958076.902 & 97.807 & 0.069 & 32.131908 & -91.084042 & 13.609 \\
\hline 416.5 & 337940 & 2066936.68 & 957525.994 & 97.868 & 0.066 & 32.130446 & -91.075170 & 13.652 \\
\hline 417 & 337898 & 2069163.95 & 956529.609 & 97.779 & 0.066 & 32.127750 & -91.067954 & 13.583 \\
\hline 417.5 & 337855 & 2071603.61 & 955788.05 & 97.979 & 0.066 & 32.125757 & -91.060058 & 13.776 \\
\hline 418 & 337810 & 2074094.43 & 954892.182 & 98.165 & 0.066 & 32.123340 & -91.051993 & 13.968 \\
\hline 418.5 & 337770 & 2076398.79 & 954590.398 & 98.371 & 0.066 & 32.122553 & -91.044544 & 14.208 \\
\hline 419 & 337731 & 2078659.93 & 954609.954 & 98.448 & 0.066 & 32.122647 & -91.037241 & 14.322 \\
\hline 419.5 & 337702 & 2080316.11 & 954943.017 & 98.613 & 0.066 & 32.123593 & -91.031899 & 14.462 \\
\hline 420 & 337669 & 2082163.29 & 955301.67 & 98.654 & 0.062 & 32.124611 & -91.025940 & 14.406 \\
\hline 420.5 & 337640 & 2083672.30 & 956057.475 & 98.696 & 0.062 & 32.126715 & -91.021082 & 14.415 \\
\hline 421 & 337607 & 2085292.00 & 957066.015 & 98.750 & 0.062 & 32.129516 & -91.015871 & 14.687 \\
\hline 421.5 & 337564 & 2086884.78 & 959011.622 & 98.908 & 0.062 & 32.134891 & -91.010765 & 14.680 \\
\hline 422 & 337518 & 2088557.69 & 961136.195 & 99.023 & 0.062 & 32.140759 & -91.005404 & 14.778 \\
\hline 422.5 & 337478 & 2089996.22 & 962877.993 & 99.180 & 0.062 & 32.145572 & -91.000792 & 14.965 \\
\hline 423 & 337409 & 2090759.46 & 966381.144 & 99.325 & 0.066 & 32.155213 & -90.998396 & 15.038 \\
\hline 423.5 & 337363 & 2090769.57 & 968991.953 & 99.393 & 0.069 & 32.162390 & -90.998415 & 15.090 \\
\hline 424 & 337314 & 2090902.51 & 971784.803 & 99.444 & 0.072 & 32.170069 & -90.998042 & 15.072 \\
\hline 424.5 & 337271 & 2091024.40 & 974291.458 & 99.399 & 0.072 & 32.176961 & -90.997698 & 15.092 \\
\hline 425 & 337228 & 2091278.59 & 976812.588 & 99.362 & 0.069 & 32.183894 & -90.996927 & 14.996 \\
\hline
\end{tabular}




\begin{tabular}{|c|c|c|c|c|c|c|c|c|}
\hline River Mile & $\begin{array}{l}\text { GPS Time } \\
\text { (seconds) }\end{array}$ & $\begin{array}{l}\text { Easting SP } \\
\quad \text { (feet) }\end{array}$ & $\begin{array}{l}\text { Northing SP } \\
\text { (feet) }\end{array}$ & $\begin{array}{l}\text { Elevation } \\
\text { Geoid } 03\end{array}$ & $\begin{array}{l}\text { Standard } \\
\text { Deviation }\end{array}$ & Latitude & Longitude & $\begin{array}{l}\text { Ellipsoid Ht } \\
\text { U.S. Survey } \\
\text { (feet) }\end{array}$ \\
\hline 425.5 & 337183 & 2091608.76 & 979461.138 & 99.663 & 0.069 & 32.191180 & -90.995912 & 15.141 \\
\hline 426 & 337138 & 2092390.26 & 982041.525 & 99.830 & 0.066 & 32.198286 & -90.993437 & 15.336 \\
\hline 426.5 & 337093 & 2093377.43 & 984514.91 & 99.967 & 0.066 & 32.205101 & -90.990295 & 15.699 \\
\hline 427 & 337051 & 2094546.67 & 986732.043 & 100.147 & 0.062 & 32.211214 & -90.986559 & 15.697 \\
\hline 427.5 & 337009 & 2095153.54 & 989132.495 & 100.100 & 0.062 & 32.217822 & -90.984644 & 15.594 \\
\hline 428 & 336962 & 2095316.14 & 991868.176 & 100.559 & 0.062 & 32.225344 & -90.984172 & 16.058 \\
\hline 428.5 & 336916 & 2095777.73 & 994538.33 & 100.763 & 0.062 & 32.232691 & -90.982731 & 16.260 \\
\hline 429 & 336873 & 2096622.61 & 996943.434 & 100.973 & 0.059 & 32.239316 & -90.980046 & 16.410 \\
\hline 429.5 & 336817 & 2098694.02 & 999313.741 & 101.078 & 0.059 & 32.245865 & -90.973393 & 16.522 \\
\hline 430 & 336759 & 2100164.54 & 1002222.548 & 101.273 & 0.059 & 32.253884 & -90.968692 & 16.675 \\
\hline 430.5 & 336715 & 2101499.76 & 1004416.556 & 101.385 & 0.059 & 32.259937 & -90.964415 & 16.821 \\
\hline 431 & 336670 & 2102952.38 & 1006615.387 & 101.404 & 0.059 & 32.266004 & -90.959758 & 16.735 \\
\hline 431.5 & 336627 & 2104396.59 & 1008760.124 & 101.539 & 0.059 & 32.271922 & -90.955126 & 17.043 \\
\hline 432 & 336584 & 2106032.68 & 1010799.198 & 101.749 & 0.059 & 32.277552 & -90.949871 & 16.944 \\
\hline 432.5 & 336542 & 2107892.39 & 1012583.975 & 101.643 & 0.059 & 32.282487 & -90.943887 & 17.143 \\
\hline 433 & 336500 & 2109887.27 & 1014213.519 & 101.714 & 0.059 & 32.286997 & -90.937462 & 16.893 \\
\hline 433.5 & 336459 & 2111853.23 & 1015783.996 & 102.089 & 0.059 & 32.291344 & -90.931129 & 17.461 \\
\hline 434 & 336417 & 2113859.87 & 1017380.403 & 102.055 & 0.059 & 32.295763 & -90.924664 & 17.344 \\
\hline 434.5 & 336374 & 2115896.22 & 1019062.509 & 102.249 & 0.059 & 32.300417 & -90.918104 & 17.482 \\
\hline 435 & 336331 & 2117838.20 & 1020875.663 & 102.035 & 0.059 & 32.305429 & -90.911851 & 17.244 \\
\hline 436 & 333341 & 2120642.65 & 1025543.641 & 103.236 & 0.089 & 32.318301 & -90.902855 & 18.500 \\
\hline 436.5 & 333283 & 2121654.63 & 1027886.265 & 103.704 & 0.095 & 32.324755 & -90.899619 & 19.013 \\
\hline 437 & 333210 & 2122170.55 & 1030816.754 & 104.625 & 0.092 & 32.332817 & -90.897999 & 19.867 \\
\hline 437.5 & 332925 & 2119838.78 & 1031567.234 & 104.609 & 0.075 & 32.334846 & -90.905561 & 18.841 \\
\hline 438 & 332515 & 2116433.83 & 1032519.164 & 104.334 & 0.075 & 32.337412 & -90.916601 & 18.552 \\
\hline 438.5 & 332440 & 2114520.96 & 1033605.823 & 104.891 & 0.089 & 32.340370 & -90.922813 & 20.123 \\
\hline 439 & 332395 & 2112094.04 & 1034420.995 & 105.206 & 0.092 & 32.342574 & -90.930686 & 20.378 \\
\hline 439.5 & 332353 & 2109705.18 & 1034909.356 & 105.367 & 0.092 & 32.343879 & -90.938429 & 20.549 \\
\hline 440 & 332310 & 2107184.57 & 1035199.468 & 105.725 & 0.092 & 32.344637 & -90.946596 & 20.910 \\
\hline 440.5 & 332266 & 2104578.92 & 1035561.806 & 105.672 & 0.095 & 32.345592 & -90.955039 & 20.706 \\
\hline 441 & 332223 & 2102028.68 & 1035869.558 & 105.793 & 0.089 & 32.346397 & -90.963302 & 21.217 \\
\hline 441.5 & 332178 & 2099361.67 & 1036426.987 & 105.912 & 0.092 & 32.347885 & -90.971948 & 21.281 \\
\hline 442 & 332134 & 2096803.82 & 1037057.131 & 105.875 & 0.092 & 32.349575 & -90.980242 & 20.957 \\
\hline
\end{tabular}




\begin{tabular}{|c|c|c|c|c|c|c|c|c|}
\hline River Mile & $\begin{array}{l}\text { GPS Time } \\
\text { (seconds) }\end{array}$ & $\begin{array}{c}\text { Easting SP } \\
\text { (feet) }\end{array}$ & $\begin{array}{l}\text { Northing SP } \\
\quad \text { (feet) }\end{array}$ & $\begin{array}{l}\text { Elevation } \\
\text { Geoid } 03\end{array}$ & $\begin{array}{l}\text { Standard } \\
\text { Deviation }\end{array}$ & Latitude & Longitude & $\begin{array}{l}\text { Ellipsoid Ht } \\
\text { U.S. Survey } \\
\text { (feet) }\end{array}$ \\
\hline 442.5 & 332089 & 2094255.53 & 1037869.089 & 106.533 & 0.095 & 32.351764 & -90.988510 & 21.781 \\
\hline 443 & 332045 & 2092033.25 & 1039184.077 & 106.738 & 0.089 & 32.355341 & -90.995732 & 21.946 \\
\hline 443.5 & 332001 & 2090247.37 & 1040921.201 & 107.256 & 0.092 & 32.360085 & -91.001550 & 22.520 \\
\hline 444 & 331959 & 2089717.06 & 1043173.271 & 107.169 & 0.092 & 32.366266 & -91.003313 & 22.415 \\
\hline 444.5 & 331922 & 2090241.74 & 1045237.632 & 106.952 & 0.092 & 32.371949 & -91.001655 & 22.194 \\
\hline 445 & 331882 & 2090804.05 & 1047474.11 & 107.238 & 0.095 & 32.378106 & -90.999879 & 22.495 \\
\hline 445.5 & 331845 & 2091415.30 & 1049477.11 & 107.360 & 0.095 & 32.383621 & -90.997940 & 22.576 \\
\hline 446 & 331805 & 2092106.52 & 1051607.346 & 107.466 & 0.092 & 32.389488 & -90.995744 & 22.700 \\
\hline 446.5 & 331757 & 2092897.98 & 1054203.24 & 107.613 & 0.089 & 32.396636 & -90.993232 & 22.772 \\
\hline 447 & 331708 & 2093038.13 & 1056984.022 & 108.026 & 0.092 & 32.404282 & -90.992833 & 23.374 \\
\hline 447.5 & 331663 & 2091742.84 & 1059217.089 & 107.902 & 0.089 & 32.410398 & -90.997075 & 23.271 \\
\hline 448 & 331618 & 2090223.15 & 1061410.834 & 107.892 & 0.092 & 32.416401 & -91.002043 & 23.214 \\
\hline 448.5 & 331572 & 2088337.69 & 1063305.96 & 107.956 & 0.085 & 32.421577 & -91.008191 & 23.298 \\
\hline 449 & 331520 & 2086500.80 & 1065269.114 & 107.810 & 0.082 & 32.426941 & -91.014184 & 22.900 \\
\hline 449.5 & 331384 & 2084202.62 & 1066568.125 & 108.741 & 0.069 & 32.430471 & -91.021659 & 23.029 \\
\hline 450 & 331235 & 2081727.39 & 1067626.102 & 108.483 & 0.069 & 32.433335 & -91.029703 & 22.720 \\
\hline 450.5 & 331082 & 2079088.84 & 1068451.006 & 108.481 & 0.069 & 32.435554 & -91.038272 & 22.834 \\
\hline 451 & 330975 & 2076663.49 & 1069304.477 & 108.887 & 0.089 & 32.437856 & -91.046151 & 24.171 \\
\hline 451.5 & 330929 & 2074289.50 & 1070327.704 & 108.809 & 0.089 & 32.440624 & -91.053867 & 23.941 \\
\hline 452 & 330880 & 2071601.49 & 1071159.841 & 108.643 & 0.089 & 32.442861 & -91.062598 & 23.960 \\
\hline 452.5 & 330834 & 2069136.89 & 1071963.469 & 108.956 & 0.085 & 32.445024 & -91.070604 & 24.265 \\
\hline 453 & 330783 & 2066872.60 & 1072948.261 & 108.878 & 0.089 & 32.447687 & -91.077966 & 24.899 \\
\hline 453.5 & 330679 & 2064522.45 & 1074388.438 & 108.923 & 0.075 & 32.451600 & -91.085616 & 24.552 \\
\hline 454 & 330581 & 2062411.11 & 1075931.657 & 109.376 & 0.085 & 32.455800 & -91.092496 & 25.057 \\
\hline 454.5 & 330531 & 2060649.40 & 1077953.194 & 110.162 & 0.095 & 32.461322 & -91.098254 & 25.508 \\
\hline 455 & 330484 & 2059077.39 & 1079994.411 & 110.658 & 0.095 & 32.466901 & -91.103398 & 26.063 \\
\hline 455.5 & 330435 & 2057536.63 & 1082181.326 & 111.205 & 0.095 & 32.472881 & -91.108444 & 26.719 \\
\hline 456 & 330389 & 2056508.54 & 1084465.881 & 111.200 & 0.095 & 32.479139 & -91.111832 & 26.623 \\
\hline 456.5 & 330341 & 2055607.67 & 1086900.997 & 111.188 & 0.089 & 32.485814 & -91.114811 & 26.679 \\
\hline 457 & 330295 & 2055153.96 & 1089201.123 & 111.335 & 0.089 & 32.492127 & -91.116337 & 26.822 \\
\hline 457.5 & 330252 & 2055839.26 & 1091425.673 & 111.114 & 0.092 & 32.498254 & -91.114167 & 26.591 \\
\hline 458 & 330205 & 2056635.40 & 1093924.621 & 111.254 & 0.089 & 32.505139 & -91.111644 & 26.869 \\
\hline 458.5 & 330160 & 2057671.28 & 1096208.503 & 111.359 & 0.089 & 32.511436 & -91.108338 & 26.740 \\
\hline
\end{tabular}




\begin{tabular}{|c|c|c|c|c|c|c|c|c|}
\hline River Mile & $\begin{array}{l}\text { GPS Time } \\
\text { (seconds) }\end{array}$ & $\begin{array}{l}\text { Easting SP } \\
\text { (feet) }\end{array}$ & $\begin{array}{l}\text { Northing SP } \\
\quad \text { (feet) }\end{array}$ & $\begin{array}{l}\text { Elevation } \\
\text { Geoid } 03\end{array}$ & $\begin{array}{l}\text { Standard } \\
\text { Deviation }\end{array}$ & Latitude & Longitude & $\begin{array}{l}\text { Ellipsoid Ht } \\
\text { U.S. Survey } \\
\quad \text { (feet) }\end{array}$ \\
\hline 459 & 330113 & 2058955.08 & 1098484.474 & 111.931 & 0.092 & 32.517717 & -91.104227 & 27.487 \\
\hline 459.5 & 330062 & 2060192.36 & 1100975.584 & 112.068 & 0.089 & 32.524588 & -91.100272 & 27.612 \\
\hline 460 & 330012 & 2060925.83 & 1103558.411 & 112.262 & 0.089 & 32.531702 & -91.097953 & 27.702 \\
\hline 460.5 & 329956 & 2060994.70 & 1106505.991 & 112.775 & 0.092 & 32.539804 & -91.097798 & 28.443 \\
\hline 461 & 329904 & 2060456.57 & 1109187.143 & 112.953 & 0.092 & 32.547162 & -91.099607 & 28.503 \\
\hline 461.5 & 329858 & 2059391.65 & 1111393.566 & 112.932 & 0.095 & 32.553205 & -91.103114 & 28.310 \\
\hline 462 & 329808 & 2058691.90 & 1113980.475 & 113.092 & 0.095 & 32.560301 & -91.105446 & 28.618 \\
\hline 462.5 & 329766 & 2058015.35 & 1116155.378 & 113.212 & 0.098 & 32.566265 & -91.107693 & 28.778 \\
\hline 463 & 329718 & 2056805.05 & 1118434.571 & 113.234 & 0.095 & 32.572505 & -91.111675 & 28.806 \\
\hline 463.5 & 329671 & 2055315.74 & 1120482.427 & 113.283 & 0.102 & 32.578103 & -91.116558 & 28.880 \\
\hline 464 & 329622 & 2053514.18 & 1122410.779 & 113.600 & 0.108 & 32.583367 & -91.122452 & 29.236 \\
\hline 464.5 & 329573 & 2051752.05 & 1124306.404 & 113.941 & 0.112 & 32.588541 & -91.128218 & 29.585 \\
\hline 465 & 329522 & 2050230.97 & 1126523.846 & 114.062 & 0.112 & 32.594604 & -91.133211 & 29.881 \\
\hline 465.5 & 329473 & 2048533.91 & 1128480.731 & 113.975 & 0.115 & 32.599947 & -91.138768 & 29.687 \\
\hline 466 & 329426 & 2047082.87 & 1130505.826 & 114.297 & 0.125 & 32.605482 & -91.143530 & 30.091 \\
\hline 466.5 & 329382 & 2046153.53 & 1132646.451 & 114.208 & 0.128 & 32.611346 & -91.146600 & 30.092 \\
\hline 467 & 329336 & 2045209.20 & 1134935.497 & 114.518 & 0.135 & 32.617617 & -91.149724 & 30.331 \\
\hline 467.5 & 329294 & 2044543.16 & 1137122.324 & 114.773 & 0.138 & 32.623613 & -91.151942 & 30.767 \\
\hline 468 & 329246 & 2044172.58 & 1139700.582 & 114.862 & 0.141 & 32.630691 & -91.153210 & 30.657 \\
\hline 468.5 & 329196 & 2044363.68 & 1142377.836 & 114.976 & 0.144 & 32.638053 & -91.152656 & 30.916 \\
\hline 469 & 329140 & 2045214.16 & 1145301.306 & 115.433 & 0.138 & 32.646106 & -91.149967 & 31.312 \\
\hline 469.5 & 329084 & 2046720.34 & 1148023.675 & 115.361 & 0.141 & 32.653619 & -91.145141 & 31.187 \\
\hline 470 & 329023 & 2048826.29 & 1150560.601 & 115.859 & 0.138 & 32.660636 & -91.138362 & 31.649 \\
\hline 470.5 & 328968 & 2051389.97 & 1152069.596 & 116.053 & 0.151 & 32.664836 & -91.130070 & 31.870 \\
\hline 471 & 328917 & 2053752.57 & 1153447.755 & 116.252 & 0.144 & 32.668672 & -91.122426 & 32.048 \\
\hline 471.5 & 328867 & 2056504.39 & 1153931.426 & 115.735 & 0.144 & 32.670057 & -91.113497 & 31.406 \\
\hline 472 & 328821 & 2058949.32 & 1154762.248 & 116.009 & 0.151 & 32.672390 & -91.105572 & 31.675 \\
\hline 472.5 & 328772 & 2061601.11 & 1155317.948 & 116.109 & 0.164 & 32.673970 & -91.096968 & 31.833 \\
\hline 473 & 328653 & 2064106.79 & 1156166.014 & 115.986 & 0.118 & 32.676350 & -91.088845 & 30.628 \\
\hline 473.5 & 328503 & 2066423.17 & 1157344.447 & 116.495 & 0.115 & 32.679634 & -91.081345 & 31.155 \\
\hline 474 & 328368 & 2068475.05 & 1158865.206 & 116.356 & 0.112 & 32.683853 & -91.074711 & 30.896 \\
\hline 474.5 & 328313 & 2070359.46 & 1160715.396 & 116.689 & 0.141 & 32.688974 & -91.068629 & 32.341 \\
\hline 475 & 328269 & 2071692.24 & 1162930.314 & 116.830 & 0.138 & 32.695087 & -91.064347 & 32.305 \\
\hline
\end{tabular}




\begin{tabular}{|c|c|c|c|c|c|c|c|c|}
\hline River Mile & $\begin{array}{l}\text { GPS Time } \\
\text { (seconds) }\end{array}$ & $\begin{array}{l}\text { Easting SP } \\
\text { (feet) }\end{array}$ & $\begin{array}{l}\text { Northing SP } \\
\quad \text { (feet) }\end{array}$ & $\begin{array}{l}\text { Elevation } \\
\text { Geoid } 03\end{array}$ & $\begin{array}{l}\text { Standard } \\
\text { Deviation }\end{array}$ & Latitude & Longitude & $\begin{array}{l}\text { Ellipsoid Ht } \\
\text { U.S. Survey } \\
\quad \text { (feet) }\end{array}$ \\
\hline 475.5 & 328226 & 2072885.58 & 1165316.067 & 117.496 & 0.135 & 32.701666 & -91.060521 & 33.117 \\
\hline 476 & 328183 & 2074029.57 & 1167631.488 & 118.204 & 0.125 & 32.708051 & -91.056854 & 33.865 \\
\hline 476.5 & 328138 & 2074640.12 & 1170149.103 & 118.708 & 0.125 & 32.714982 & -91.054925 & 34.104 \\
\hline 477 & 328096 & 2074147.92 & 1172482.267 & 118.938 & 0.121 & 32.721385 & -91.056577 & 34.435 \\
\hline 477.5 & 328057 & 2072987.14 & 1174375.956 & 118.944 & 0.121 & 32.726568 & -91.060393 & 34.497 \\
\hline 478 & 328014 & 2071071.67 & 1175895.345 & 118.989 & 0.121 & 32.730708 & -91.066656 & 34.578 \\
\hline 478.5 & 327973 & 2068905.73 & 1176883.758 & 118.781 & 0.118 & 32.733383 & -91.073721 & 34.370 \\
\hline 479 & 327930 & 2066410.09 & 1177362.316 & 118.776 & 0.128 & 32.734650 & -91.081847 & 34.368 \\
\hline 479.5 & 327888 & 2063814.71 & 1177372.953 & 118.837 & 0.128 & 32.734628 & -91.090286 & 34.391 \\
\hline 480 & 327847 & 2061262.61 & 1177352.71 & 118.832 & 0.128 & 32.734522 & -91.098585 & 34.377 \\
\hline 480.5 & 327803 & 2058535.38 & 1177144.584 & 119.159 & 0.131 & 32.733896 & -91.107448 & 34.881 \\
\hline 481 & 327758 & 2055780.88 & 1176895.656 & 119.729 & 0.135 & 32.733156 & -91.116398 & 35.422 \\
\hline 481.5 & 327714 & 2053110.33 & 1176854.118 & 120.113 & 0.135 & 32.732987 & -91.125081 & 35.825 \\
\hline 482 & 327665 & 2050448.63 & 1176961.425 & 120.474 & 0.138 & 32.733227 & -91.133739 & 36.286 \\
\hline 482.5 & 327620 & 2048147.96 & 1177581.403 & 120.899 & 0.135 & 32.734883 & -91.141235 & 36.720 \\
\hline 483 & 327575 & 2046409.09 & 1179179.836 & 120.801 & 0.138 & 32.739240 & -91.146929 & 36.701 \\
\hline 483.5 & 327530 & 2045293.71 & 1181272.255 & 120.914 & 0.141 & 32.744967 & -91.150609 & 36.919 \\
\hline 484 & 327480 & 2044360.84 & 1183687.734 & 120.817 & 0.125 & 32.751585 & -91.153703 & 36.609 \\
\hline 484.5 & 327405 & 2043448.50 & 1186420.621 & 120.909 & 0.102 & 32.759076 & -91.156739 & 35.962 \\
\hline 485 & 327351 & 2043109.90 & 1188862.097 & 121.161 & 0.118 & 32.765779 & -91.157903 & 37.080 \\
\hline 485.5 & 327301 & 2043072.73 & 1191692.126 & 121.289 & 0.128 & 32.773556 & -91.158095 & 37.179 \\
\hline 486 & 327254 & 2042968.62 & 1194457.861 & 121.582 & 0.128 & 32.781155 & -91.158504 & 37.381 \\
\hline 486.5 & 327211 & 2043056.35 & 1196974.93 & 121.854 & 0.121 & 32.788074 & -91.158282 & 37.609 \\
\hline 487 & 327165 & 2043484.52 & 1199634.899 & 122.040 & 0.115 & 32.795394 & -91.156957 & 38.001 \\
\hline 487.5 & 327120 & 2044160.47 & 1202200.789 & 122.083 & 0.115 & 32.802460 & -91.154822 & 37.860 \\
\hline 488 & 327075 & 2044651.55 & 1204782.578 & 122.241 & 0.112 & 32.809566 & -91.153289 & 38.068 \\
\hline 488.5 & 327033 & 2045443.36 & 1207124.248 & 122.563 & 0.105 & 32.816018 & -91.150771 & 38.492 \\
\hline 489 & 326993 & 2046263.83 & 1209315.943 & 122.763 & 0.108 & 32.822059 & -91.148156 & 38.571 \\
\hline 489.5 & 326945 & 2047166.89 & 1211991.588 & 123.075 & 0.105 & 32.829431 & -91.145284 & 38.850 \\
\hline 490 & 326890 & 2048695.85 & 1214781.212 & 123.149 & 0.102 & 32.837130 & -91.140376 & 38.915 \\
\hline 490.5 & 326841 & 2050592.46 & 1216808.627 & 123.303 & 0.105 & 32.842742 & -91.134252 & 39.067 \\
\hline 491 & 326794 & 2052603.49 & 1218550.602 & 123.600 & 0.098 & 32.847571 & -91.127747 & 39.340 \\
\hline 491.5 & 326746 & 2054737.28 & 1220247.394 & 123.785 & 0.095 & 32.852278 & -91.120841 & 39.529 \\
\hline
\end{tabular}




\begin{tabular}{|c|c|c|c|c|c|c|c|c|}
\hline River Mile & $\begin{array}{l}\text { GPS Time } \\
\text { (seconds) }\end{array}$ & $\begin{array}{l}\text { Easting SP } \\
\text { (feet) }\end{array}$ & $\begin{array}{l}\text { Northing SP } \\
\quad \text { (feet) }\end{array}$ & $\begin{array}{l}\text { Elevation } \\
\text { Geoid } 03\end{array}$ & $\begin{array}{l}\text { Standard } \\
\text { Deviation }\end{array}$ & Latitude & Longitude & $\begin{array}{l}\text { Ellipsoid Ht } \\
\text { U.S. Survey } \\
\quad \text { (feet) }\end{array}$ \\
\hline 492 & 326698 & 2056880.09 & 1221909.501 & 123.945 & 0.092 & 32.856889 & -91.113904 & 39.645 \\
\hline 492.5 & 326656 & 2058754.18 & 1223400.071 & 124.023 & 0.089 & 32.861024 & -91.107838 & 39.722 \\
\hline 493 & 326606 & 2060941.34 & 1225188.713 & 124.146 & 0.085 & 32.865983 & -91.100758 & 39.784 \\
\hline 493.5 & 326561 & 2063116.94 & 1226498.61 & 124.168 & 0.082 & 32.869627 & -91.093704 & 39.757 \\
\hline 494 & 326517 & 2064962.39 & 1228222.876 & 124.347 & 0.075 & 32.874402 & -91.087734 & 40.103 \\
\hline 494.5 & 326473 & 2066755.46 & 1230111.016 & 124.512 & 0.075 & 32.879626 & -91.081938 & 40.175 \\
\hline 495 & 326430 & 2068552.30 & 1231937.625 & 124.875 & 0.072 & 32.884681 & -91.076128 & 40.458 \\
\hline 495.5 & 326385 & 2070209.33 & 1234022.489 & 124.989 & 0.075 & 32.890443 & -91.070778 & 40.566 \\
\hline 496 & 326339 & 2071691.15 & 1236313.465 & 125.286 & 0.072 & 32.896767 & -91.066003 & 41.005 \\
\hline 496.5 & 326290 & 2072786.17 & 1238976.266 & 125.396 & 0.072 & 32.904106 & -91.062495 & 41.016 \\
\hline 497 & 326242 & 2072696.50 & 1241849.582 & 125.305 & 0.072 & 32.912001 & -91.062852 & 41.047 \\
\hline 497.5 & 326200 & 2072444.80 & 1244400.972 & 125.628 & 0.072 & 32.919008 & -91.063730 & 41.450 \\
\hline 498 & 326155 & 2071762.41 & 1246994.239 & 126.040 & 0.069 & 32.926122 & -91.066012 & 41.698 \\
\hline 498.5 & 326112 & 2070609.42 & 1249291.907 & 126.296 & 0.072 & 32.932415 & -91.069822 & 42.124 \\
\hline 499 & 326067 & 2069231.41 & 1251598.52 & 126.514 & 0.072 & 32.938727 & -91.074366 & 42.375 \\
\hline 499.5 & 326023 & 2067312.05 & 1253460.303 & 126.584 & 0.075 & 32.943807 & -91.080664 & 42.393 \\
\hline 500 & 325976 & 2064824.79 & 1254830.349 & 126.626 & 0.079 & 32.947523 & -91.088803 & 42.510 \\
\hline 500.5 & 325934 & 2062418.50 & 1255685.647 & 126.685 & 0.082 & 32.949826 & -91.096666 & 42.606 \\
\hline 501 & 325892 & 2060000.17 & 1256338.42 & 126.927 & 0.082 & 32.951572 & -91.104565 & 42.832 \\
\hline 501.5 & 325848 & 2057440.63 & 1256950.461 & 127.128 & 0.089 & 32.953202 & -91.112923 & 43.039 \\
\hline 502 & 325804 & 2054861.30 & 1257484.84 & 127.407 & 0.089 & 32.954618 & -91.121343 & 43.482 \\
\hline 502.5 & 325757 & 2052154.96 & 1258107.867 & 127.760 & 0.092 & 32.956274 & -91.130181 & 43.789 \\
\hline 503 & 325708 & 2049389.48 & 1258571.806 & 128.297 & 0.092 & 32.957491 & -91.139207 & 44.327 \\
\hline 503.5 & 325652 & 2046331.87 & 1259187.924 & 128.799 & 0.21 & 32.959120 & -91.149190 & 44.916 \\
\hline 504 & 325607 & 2044532.29 & 1260867.349 & 129.085 & 0.217 & 32.963697 & -91.155099 & 45.230 \\
\hline 504.5 & 325568 & 2043592.43 & 1262801.012 & 129.013 & 0.207 & 32.968990 & -91.158212 & 45.200 \\
\hline 505 & 325520 & 2043209.68 & 1265408.173 & 128.883 & 0.207 & 32.976147 & -91.159527 & 45.090 \\
\hline 505.5 & 325472 & 2042774.76 & 1267997.181 & 129.069 & 0.213 & 32.983253 & -91.161011 & 45.320 \\
\hline 506 & 325424 & 2042750.76 & 1270654.967 & 129.009 & 0.203 & 32.990556 & -91.161158 & 45.310 \\
\hline 506.5 & 325385 & 2043303.86 & 1272742.772 & 128.693 & 0.207 & 32.996306 & -91.159407 & 44.945 \\
\hline 507 & 325346 & 2043952.10 & 1275026.028 & 129.173 & 0.21 & 33.002595 & -91.157352 & 45.440 \\
\hline 507.5 & 325304 & 2044655.25 & 1277506.882 & 129.698 & 0.203 & 33.009428 & -91.155122 & 46.172 \\
\hline 508 & 325258 & 2045393.65 & 1280211.039 & 130.002 & 0.21 & 33.016875 & -91.152782 & 46.427 \\
\hline
\end{tabular}




\begin{tabular}{|c|c|c|c|c|c|c|c|c|}
\hline River Mile & $\begin{array}{l}\text { GPS Time } \\
\text { (seconds) }\end{array}$ & $\begin{array}{l}\text { Easting SP } \\
\text { (feet) }\end{array}$ & $\begin{array}{l}\text { Northing SP } \\
\quad \text { (feet) }\end{array}$ & $\begin{array}{l}\text { Elevation } \\
\text { Geoid } 03\end{array}$ & $\begin{array}{l}\text { Standard } \\
\text { Deviation }\end{array}$ & Latitude & Longitude & $\begin{array}{l}\text { Ellipsoid Ht } \\
\text { U.S. Survey } \\
\quad \text { (feet) }\end{array}$ \\
\hline 508.5 & 325207 & 2045977.35 & 1283220.176 & 130.548 & 0.21 & 33.025157 & -91.150954 & 47.067 \\
\hline 509 & 325165 & 2046113.52 & 1285760.508 & 130.506 & 0.207 & 33.032141 & -91.150574 & 46.923 \\
\hline 509.5 & 325118 & 2045715.97 & 1288492.446 & 130.609 & 0.207 & 33.039640 & -91.151941 & 47.050 \\
\hline 510 & 325075 & 2044943.09 & 1290867.948 & 130.486 & 0.203 & 33.046152 & -91.154523 & 46.888 \\
\hline 510.5 & 325031 & 2043845.10 & 1293238.2 & 130.750 & 0.213 & 33.052642 & -91.158167 & 47.241 \\
\hline 511 & 324986 & 2042880.05 & 1295836.308 & 131.098 & 0.223 & 33.059761 & -91.161383 & 47.593 \\
\hline 511.5 & 324944 & 2041939.04 & 1298300.278 & 131.350 & 0.203 & 33.066512 & -91.164518 & 47.882 \\
\hline 512 & 324898 & 2041060.56 & 1301024.311 & 131.901 & 0.207 & 33.073979 & -91.167455 & 48.427 \\
\hline 512.5 & 324853 & 2040488.56 & 1303699.094 & 132.641 & 0.194 & 33.081317 & -91.169392 & 49.234 \\
\hline 513 & 324805 & 2040245.77 & 1306473.256 & 133.265 & 0.184 & 33.088936 & -91.170257 & 49.864 \\
\hline 513.5 & 324756 & 2041176.89 & 1309106.616 & 133.424 & 0.187 & 33.096193 & -91.167285 & 50.030 \\
\hline 514 & 324707 & 2042835.73 & 1311352.888 & 133.618 & 0.18 & 33.102402 & -91.161927 & 50.230 \\
\hline 514.5 & 324658 & 2044957.46 & 1313113.318 & 133.669 & 0.18 & 33.107286 & -91.155045 & 50.276 \\
\hline 515 & 324613 & 2047333.74 & 1314354.422 & 133.668 & 0.174 & 33.110747 & -91.147317 & 50.212 \\
\hline 515.5 & 324570 & 2049778.62 & 1315275.126 & 133.637 & 0.174 & 33.113329 & -91.139356 & 50.254 \\
\hline 516 & 324528 & 2052267.43 & 1315825.265 & 133.835 & 0.164 & 33.114894 & -91.131242 & 50.357 \\
\hline 516.5 & 324482 & 2054984.50 & 1316328.637 & 134.161 & 0.161 & 33.116333 & -91.122382 & 50.700 \\
\hline 517 & 324436 & 2057662.00 & 1316752.646 & 134.483 & 0.151 & 33.117554 & -91.113648 & 50.901 \\
\hline 517.5 & 324393 & 2060090.48 & 1317496.093 & 134.797 & 0.154 & 33.119646 & -91.105736 & 51.319 \\
\hline 518 & 324353 & 2062017.72 & 1318897.969 & 134.819 & 0.141 & 33.123538 & -91.099475 & 51.358 \\
\hline 518.5 & 324313 & 2063604.66 & 1320669.274 & 134.962 & 0.128 & 33.128437 & -91.094334 & 51.475 \\
\hline 519 & 324272 & 2064873.75 & 1322799.936 & 135.125 & 0.131 & 33.134318 & -91.090239 & 51.368 \\
\hline 519.5 & 324232 & 2065916.14 & 1324992.517 & 135.193 & 0.121 & 33.140364 & -91.086886 & 51.489 \\
\hline 520 & 324191 & 2066729.31 & 1327347.754 & 135.395 & 0.118 & 33.146853 & -91.084285 & 51.758 \\
\hline 520.5 & 324146 & 2067250.96 & 1330041.643 & 135.561 & 0.105 & 33.154266 & -91.082644 & 51.912 \\
\hline 521 & 324100 & 2067452.43 & 1332826.846 & 135.768 & 0.102 & 33.161924 & -91.082050 & 52.062 \\
\hline 521.5 & 324059 & 2067588.11 & 1335324.78 & 135.941 & 0.105 & 33.168792 & -91.081665 & 52.224 \\
\hline 522 & 324017 & 2067480.89 & 1337868.391 & 136.171 & 0.102 & 33.175780 & -91.082075 & 52.509 \\
\hline 522.5 & 323975 & 2066936.96 & 1340385.213 & 136.143 & 0.095 & 33.182686 & -91.083912 & 52.461 \\
\hline 523 & 323935 & 2066173.34 & 1342740.599 & 136.336 & 0.098 & 33.189143 & -91.086463 & 52.730 \\
\hline 523.5 & 323891 & 2065109.06 & 1345258.926 & 136.378 & 0.138 & 33.196043 & -91.090001 & 52.647 \\
\hline 524 & 323845 & 2063995.61 & 1347859.935 & 136.685 & 0.098 & 33.203169 & -91.093702 & 52.916 \\
\hline 524.5 & 323801 & 2062994.31 & 1350360.27 & 136.821 & 0.098 & 33.210020 & -91.097035 & 53.038 \\
\hline
\end{tabular}




\begin{tabular}{|c|c|c|c|c|c|c|c|c|}
\hline River Mile & $\begin{array}{l}\text { GPS Time } \\
\text { (seconds) }\end{array}$ & $\begin{array}{l}\text { Easting SP } \\
\text { (feet) }\end{array}$ & $\begin{array}{l}\text { Northing SP } \\
\quad \text { (feet) }\end{array}$ & $\begin{array}{l}\text { Elevation } \\
\text { Geoid } 03\end{array}$ & $\begin{array}{l}\text { Standard } \\
\text { Deviation }\end{array}$ & Latitude & Longitude & $\begin{array}{l}\text { Ellipsoid Ht } \\
\text { U.S. Survey } \\
\quad \text { (feet) }\end{array}$ \\
\hline 525 & 323757 & 2062091.64 & 1352816.905 & 137.013 & 0.098 & 33.216753 & -91.100045 & 53.221 \\
\hline 525.5 & 323711 & 2061313.98 & 1355263.71 & 137.486 & 0.092 & 33.223461 & -91.102646 & 53.642 \\
\hline 526 & 323666 & 2060110.90 & 1357490.043 & 137.750 & 0.095 & 33.229555 & -91.106633 & 53.893 \\
\hline 526.5 & 323619 & 2058924.58 & 1359856.607 & 137.932 & 0.095 & 33.236034 & -91.110570 & 54.130 \\
\hline 527 & 323574 & 2057760.39 & 1362128.506 & 137.982 & 0.092 & 33.242254 & -91.114433 & 54.159 \\
\hline 527.5 & 323523 & 2056230.54 & 1364549.728 & 138.068 & 0.092 & 33.248876 & -91.119495 & 54.263 \\
\hline 528 & 323474 & 2054942.22 & 1366989.858 & 138.186 & 0.085 & 33.255555 & -91.123769 & 54.349 \\
\hline 528.5 & 323427 & 2053514.12 & 1369252.441 & 138.324 & 0.089 & 33.261743 & -91.128497 & 54.473 \\
\hline 529 & 323382 & 2052032.30 & 1371396.264 & 138.264 & 0.089 & 33.267603 & -91.133398 & 54.466 \\
\hline 529.5 & 323336 & 2050541.32 & 1373654.198 & 138.616 & 0.085 & 33.273777 & -91.138332 & 54.882 \\
\hline 530 & 323291 & 2048675.43 & 1375621.026 & 138.220 & 0.089 & 33.279142 & -91.144486 & 54.334 \\
\hline 530.5 & 323248 & 2046862.35 & 1377412.47 & 138.502 & 0.089 & 33.284026 & -91.150464 & 54.690 \\
\hline 531 & 323201 & 2045009.52 & 1379245.631 & 138.397 & 0.085 & 33.289024 & -91.156574 & 54.615 \\
\hline 531.5 & 322873 & 2043394.24 & 1381300.192 & 139.070 & 0.082 & 33.294635 & -91.161912 & 55.228 \\
\hline 532 & 322831 & 2041781.27 & 1383291.345 & 139.694 & 0.085 & 33.300071 & -91.167242 & 55.822 \\
\hline 532.5 & 322791 & 2040344.85 & 1385151.28 & 140.213 & 0.082 & 33.305151 & -91.171992 & 56.377 \\
\hline 533 & 322743 & 2038840.34 & 1387370.484 & 140.746 & 0.079 & 33.311216 & -91.176974 & 56.893 \\
\hline 533.5 & 322689 & 2037969.46 & 1390207.813 & 141.247 & 0.079 & 33.318993 & -91.179900 & 57.381 \\
\hline 534 & 322595 & 2039711.77 & 1394930.694 & 141.427 & 0.079 & 33.332011 & -91.174322 & 57.452 \\
\hline 534.5 & 322530 & 2042302.55 & 1397426.246 & 141.509 & 0.079 & 33.338926 & -91.165906 & 57.434 \\
\hline 535 & 322477 & 2044798.87 & 1398987.566 & 141.594 & 0.082 & 33.343271 & -91.157773 & 57.493 \\
\hline 535.5 & 322430 & 2047021.82 & 1400405.091 & 141.584 & 0.079 & 33.347214 & -91.150532 & 57.410 \\
\hline 536 & 322382 & 2049483.09 & 1401585.179 & 141.644 & 0.079 & 33.350510 & -91.142503 & 57.433 \\
\hline 536.5 & 322340 & 2051677.13 & 1402698.053 & 142.044 & 0.082 & 33.353615 & -91.135347 & 57.770 \\
\hline 537 & 322296 & 2053868.31 & 1404189.286 & 142.193 & 0.075 & 33.357759 & -91.128209 & 57.905 \\
\hline 537.5 & 322252 & 2055746.45 & 1406049.441 & 142.472 & 0.079 & 33.362910 & -91.122105 & 58.102 \\
\hline 538 & 322208 & 2057538.73 & 1407945.682 & 142.939 & 0.079 & 33.368158 & -91.116282 & 58.518 \\
\hline 538.5 & 322164 & 2058781.56 & 1410237.228 & 143.133 & 0.079 & 33.374481 & -91.112268 & 58.733 \\
\hline 539 & 322121 & 2059356.59 & 1412680.54 & 143.253 & 0.079 & 33.381207 & -91.110444 & 58.790 \\
\hline 539.5 & 322074 & 2059203.84 & 1415358.169 & 143.349 & 0.082 & 33.388562 & -91.111010 & 58.803 \\
\hline 540 & 322024 & 2058627.25 & 1418132.943 & 143.575 & 0.082 & 33.396175 & -91.112967 & 59.156 \\
\hline 540.5 & 321981 & 2058854.17 & 1420565.069 & 143.881 & 0.075 & 33.402863 & -91.112283 & 59.378 \\
\hline 541 & 321937 & 2059194.26 & 1423013.343 & 144.009 & 0.079 & 33.409598 & -91.111229 & 59.514 \\
\hline
\end{tabular}




\begin{tabular}{|c|c|c|c|c|c|c|c|c|}
\hline River Mile & $\begin{array}{l}\text { GPS Time } \\
\text { (seconds) }\end{array}$ & $\begin{array}{c}\text { Easting SP } \\
\text { (feet) }\end{array}$ & $\begin{array}{l}\text { Northing SP } \\
\quad \text { (feet) }\end{array}$ & $\begin{array}{l}\text { Elevation } \\
\text { Geoid } 03\end{array}$ & $\begin{array}{l}\text { Standard } \\
\text { Deviation }\end{array}$ & Latitude & Longitude & $\begin{array}{l}\text { Ellipsoid Ht } \\
\text { U.S. Survey } \\
\text { (feet) }\end{array}$ \\
\hline 541.5 & 321892 & 2058894.24 & 1425503.198 & 144.118 & 0.079 & 33.416434 & -91.112273 & 59.523 \\
\hline 542 & 321844 & 2058317.99 & 1428092.432 & 144.140 & 0.079 & 33.423538 & -91.114225 & 59.655 \\
\hline 542.5 & 321795 & 2057872.11 & 1430755.437 & 144.267 & 0.075 & 33.430846 & -91.115752 & 59.627 \\
\hline 543 & 321745 & 2057415.64 & 1433385.167 & 144.494 & 0.079 & 33.438064 & -91.117313 & 59.922 \\
\hline 543.5 & 321696 & 2057121.01 & 1436066.309 & 144.599 & 0.075 & 33.445425 & -91.118345 & 59.999 \\
\hline 544 & 321645 & 2056935.71 & 1438708.497 & 144.705 & 0.075 & 33.452682 & -91.119018 & 59.994 \\
\hline 544.5 & 321595 & 2056730.00 & 1441216.701 & 144.956 & 0.075 & 33.459570 & -91.119754 & 60.314 \\
\hline 545 & 321537 & 2056198.82 & 1443900.735 & 145.250 & 0.075 & 33.466935 & -91.121563 & 60.627 \\
\hline 545.5 & 321469 & 2054916.45 & 1446625.44 & 145.102 & 0.075 & 33.474396 & -91.125835 & 60.445 \\
\hline 546 & 321417 & 2053619.80 & 1448926.859 & 145.508 & 0.075 & 33.480693 & -91.130145 & 60.995 \\
\hline 546.5 & 321342 & 2051217.17 & 1451483.412 & 145.645 & 0.075 & 33.487667 & -91.138089 & 61.094 \\
\hline 547 & 321280 & 2048761.35 & 1453145.246 & 145.804 & 0.075 & 33.492181 & -91.146185 & 61.328 \\
\hline 547.5 & 321200 & 2046468.52 & 1454330.038 & 145.763 & 0.066 & 33.495387 & -91.153736 & 60.144 \\
\hline 548 & 321065 & 2044152.57 & 1455920.608 & 145.933 & 0.069 & 33.499708 & -91.161373 & 60.379 \\
\hline 548.5 & 320937 & 2041566.60 & 1457404.721 & 145.805 & 0.072 & 33.503729 & -91.169894 & 61.356 \\
\hline 549 & 320844 & 2038826.06 & 1458574.014 & 146.371 & 0.089 & 33.506881 & -91.178915 & 62.160 \\
\hline 549.5 & 320801 & 2036480.72 & 1459505.002 & 146.419 & 0.092 & 33.509386 & -91.186633 & 61.823 \\
\hline 550 & 320758 & 2034214.61 & 1460654.426 & 146.545 & 0.092 & 33.512494 & -91.194098 & 62.048 \\
\hline 550.5 & 320715 & 2032033.35 & 1461945.14 & 147.035 & 0.095 & 33.515990 & -91.201289 & 62.607 \\
\hline 551 & 320671 & 2029946.62 & 1463472.292 & 147.167 & 0.102 & 33.520139 & -91.208177 & 62.800 \\
\hline 551.5 & 320627 & 2027922.74 & 1465167.486 & 147.206 & 0.105 & 33.524750 & -91.214864 & 62.856 \\
\hline 552 & 320582 & 2026144.04 & 1467264.444 & 147.388 & 0.108 & 33.530470 & -91.220758 & 63.060 \\
\hline 552.5 & 320535 & 2024589.18 & 1469624.974 & 147.386 & 0.105 & 33.536920 & -91.225927 & 63.044 \\
\hline 553 & 320491 & 2023436.23 & 1472004.009 & 147.860 & 0.112 & 33.543430 & -91.229777 & 63.523 \\
\hline 553.5 & 320411 & 2022270.00 & 1474475.709 & 148.755 & 0.082 & 33.550194 & -91.233675 & 63.415 \\
\hline 554 & 319983 & 2022283.88 & 1478360.451 & 149.735 & 0.112 & 33.560869 & -91.233740 & 65.466 \\
\hline 554.5 & 319947 & 2023609.59 & 1479949.387 & 149.629 & 0.105 & 33.565267 & -91.229434 & 65.260 \\
\hline 555 & 319900 & 2025918.30 & 1481447.977 & 149.718 & 0.108 & 33.569440 & -91.221898 & 65.338 \\
\hline 555.5 & 319852 & 2028691.73 & 1481974.115 & 149.914 & 0.112 & 33.570951 & -91.212809 & 65.462 \\
\hline 556 & 319806 & 2031427.14 & 1481878.757 & 150.120 & 0.105 & 33.570752 & -91.203827 & 65.659 \\
\hline 556.5 & 319762 & 2034026.52 & 1481685.103 & 150.357 & 0.105 & 33.570280 & -91.195289 & 65.892 \\
\hline 557 & 319719 & 2036499.36 & 1481520.889 & 150.587 & 0.105 & 33.569885 & -91.187167 & 66.056 \\
\hline 557.5 & 319674 & 2039033.33 & 1481683.467 & 150.791 & 0.102 & 33.570388 & -91.178853 & 66.250 \\
\hline
\end{tabular}




\begin{tabular}{|c|c|c|c|c|c|c|c|c|}
\hline River Mile & $\begin{array}{l}\text { GPS Time } \\
\text { (seconds) }\end{array}$ & $\begin{array}{l}\text { Easting SP } \\
\text { (feet) }\end{array}$ & $\begin{array}{l}\text { Northing SP } \\
\quad \text { (feet) }\end{array}$ & $\begin{array}{l}\text { Elevation } \\
\text { Geoid } 03\end{array}$ & $\begin{array}{l}\text { Standard } \\
\text { Deviation }\end{array}$ & Latitude & Longitude & $\begin{array}{l}\text { Ellipsoid Ht } \\
\text { U.S. Survey } \\
\quad \text { (feet) }\end{array}$ \\
\hline 558 & 319627 & 2041630.66 & 1482240.36 & 151.065 & 0.102 & 33.571977 & -91.170342 & 66.502 \\
\hline 558.5 & 319585 & 2043972.13 & 1482929.45 & 151.169 & 0.105 & 33.573922 & -91.162674 & 66.603 \\
\hline 559 & 319541 & 2046279.29 & 1484094.342 & 151.233 & 0.108 & 33.577174 & -91.155130 & 66.664 \\
\hline 559.5 & 319497 & 2048436.86 & 1485567.036 & 151.439 & 0.105 & 33.581267 & -91.148085 & 66.909 \\
\hline 560 & 319453 & 2050366.76 & 1487338.744 & 151.560 & 0.105 & 33.586177 & -91.141794 & 66.881 \\
\hline 560.5 & 319406 & 2052141.81 & 1489492.711 & 151.929 & 0.105 & 33.592134 & -91.136021 & 67.266 \\
\hline 561 & 319358 & 2053552.02 & 1491897.282 & 152.177 & 0.108 & 33.598772 & -91.131452 & 67.482 \\
\hline 561.5 & 319313 & 2054030.78 & 1494444.741 & 152.277 & 0.108 & 33.605782 & -91.129944 & 67.600 \\
\hline 562 & 319270 & 2053691.05 & 1496902.981 & 152.488 & 0.108 & 33.612530 & -91.131122 & 67.783 \\
\hline 562.5 & 319223 & 2052834.92 & 1499461.533 & 152.665 & 0.108 & 33.619542 & -91.133999 & 67.997 \\
\hline 563 & 319178 & 2051477.26 & 1501643.941 & 152.757 & 0.115 & 33.625510 & -91.138514 & 68.093 \\
\hline 563.5 & 319134 & 2049759.75 & 1503465.408 & 152.840 & 0.105 & 33.630479 & -91.144202 & 68.188 \\
\hline 564 & 319090 & 2047820.12 & 1505121.308 & 152.817 & 0.112 & 33.634987 & -91.150617 & 68.200 \\
\hline 564.5 & 319039 & 2045482.09 & 1507090.211 & 152.747 & 0.115 & 33.640346 & -91.158349 & 68.143 \\
\hline 565 & 318991 & 2043318.17 & 1509018.922 & 152.964 & 0.112 & 33.645599 & -91.165509 & 68.373 \\
\hline 565.5 & 318951 & 2041441.64 & 1510391.373 & 153.182 & 0.118 & 33.649328 & -91.171711 & 68.617 \\
\hline 566 & 318916 & 2039570.87 & 1511204.862 & 153.102 & 0.125 & 33.651522 & -91.177879 & 68.562 \\
\hline 566.5 & 318874 & 2037088.93 & 1511959.734 & 153.176 & 0.118 & 33.653540 & -91.186055 & 68.641 \\
\hline 567 & 318832 & 2034555.45 & 1512670.24 & 153.409 & 0.121 & 33.655435 & -91.194399 & 68.921 \\
\hline 567.5 & 318789 & 2032011.55 & 1513429.273 & 153.722 & 0.121 & 33.657462 & -91.202778 & 69.142 \\
\hline 568 & 318744 & 2029401.40 & 1514137.337 & 154.093 & 0.115 & 33.659347 & -91.211375 & 69.495 \\
\hline 568.5 & 318701 & 2027117.59 & 1515187.817 & 154.832 & 0.118 & 33.662180 & -91.218909 & 70.409 \\
\hline 569 & 318646 & 2024737.69 & 1517199.931 & 155.384 & 0.118 & 33.667653 & -91.226787 & 71.027 \\
\hline 569.5 & 318592 & 2024194.10 & 1520109.429 & 155.741 & 0.115 & 33.675635 & -91.228656 & 71.413 \\
\hline 570 & 318539 & 2024351.19 & 1522748.153 & 156.053 & 0.118 & 33.682889 & -91.228215 & 71.673 \\
\hline 570.5 & 318484 & 2024434.93 & 1525243.629 & 156.132 & 0.115 & 33.689748 & -91.228011 & 71.753 \\
\hline 571 & 318150 & 2027066.33 & 1528176.025 & 156.311 & 0.118 & 33.697868 & -91.219443 & 71.923 \\
\hline 571.5 & 318097 & 2029551.65 & 1529512.396 & 156.314 & 0.115 & 33.701599 & -91.211311 & 71.885 \\
\hline 572 & 318042 & 2032295.76 & 1530196.444 & 156.378 & 0.115 & 33.703542 & -91.202308 & 71.944 \\
\hline 572.5 & 317996 & 2034914.01 & 1529936.059 & 156.400 & 0.112 & 33.702887 & -91.193693 & 71.902 \\
\hline 573 & 317955 & 2037299.65 & 1529553.369 & 156.706 & 0.115 & 33.701890 & -91.185839 & 72.170 \\
\hline 573.5 & 317911 & 2039869.97 & 1529540.495 & 157.161 & 0.108 & 33.701912 & -91.177389 & 72.595 \\
\hline 574 & 317862 & 2042561.56 & 1530120.895 & 157.473 & 0.105 & 33.703567 & -91.168555 & 72.939 \\
\hline
\end{tabular}




\begin{tabular}{|c|c|c|c|c|c|c|c|c|}
\hline River Mile & $\begin{array}{l}\text { GPS Time } \\
\text { (seconds) }\end{array}$ & $\begin{array}{l}\text { Easting SP } \\
\text { (feet) }\end{array}$ & $\begin{array}{l}\text { Northing SP } \\
\quad \text { (feet) }\end{array}$ & $\begin{array}{l}\text { Elevation } \\
\text { Geoid } 03\end{array}$ & $\begin{array}{l}\text { Standard } \\
\text { Deviation }\end{array}$ & Latitude & Longitude & $\begin{array}{l}\text { Ellipsoid Ht } \\
\text { U.S. Survey } \\
\quad \text { (feet) }\end{array}$ \\
\hline 574.5 & 317813 & 2044905.88 & 1531429.013 & 157.681 & 0.098 & 33.707214 & -91.160882 & 73.148 \\
\hline 575 & 317763 & 2046792.54 & 1533415.291 & 157.653 & 0.102 & 33.712713 & -91.154731 & 73.072 \\
\hline 575.5 & 317718 & 2047982.55 & 1535654.465 & 157.645 & 0.098 & 33.718892 & -91.150877 & 73.111 \\
\hline 576 & 317676 & 2048453.33 & 1538032.823 & 157.971 & 0.108 & 33.725438 & -91.149391 & 73.435 \\
\hline 576.5 & 317631 & 2048926.32 & 1540645.834 & 158.329 & 0.102 & 33.732628 & -91.147903 & 73.791 \\
\hline 577 & 317588 & 2049613.32 & 1543127.633 & 158.715 & 0.102 & 33.739462 & -91.145708 & 74.183 \\
\hline 577.5 & 317542 & 2050884.28 & 1545534.393 & 158.952 & 0.102 & 33.746103 & -91.141590 & 74.429 \\
\hline 578 & 317497 & 2052617.85 & 1547503.54 & 159.192 & 0.102 & 33.751551 & -91.135938 & 74.525 \\
\hline 578.5 & 317451 & 2054526.77 & 1549430.176 & 159.478 & 0.098 & 33.756886 & -91.129707 & 74.840 \\
\hline 579 & 317401 & 2056664.83 & 1551108.652 & 159.566 & 0.095 & 33.761544 & -91.122716 & 75.035 \\
\hline 579.5 & 317358 & 2058419.71 & 1552574.426 & 159.854 & 0.098 & 33.765608 & -91.116979 & 75.279 \\
\hline 580 & 317272 & 2060804.49 & 1553856.859 & 160.049 & 0.082 & 33.769182 & -91.109165 & 74.509 \\
\hline 580.5 & 317188 & 2063166.17 & 1554834.099 & 160.472 & 0.095 & 33.771916 & -91.101418 & 75.846 \\
\hline 581 & 317140 & 2065765.01 & 1555658.573 & 160.521 & 0.095 & 33.774234 & -91.092887 & 75.854 \\
\hline 581.5 & 317094 & 2068400.54 & 1555953.646 & 160.631 & 0.092 & 33.775098 & -91.084222 & 75.958 \\
\hline 582 & 317048 & 2071044.00 & 1556066.638 & 160.826 & 0.092 & 33.775461 & -91.075526 & 76.125 \\
\hline 582.5 & 317002 & 2073678.97 & 1556297.835 & 161.031 & 0.089 & 33.776148 & -91.066861 & 76.342 \\
\hline 583 & 316955 & 2076309.37 & 1556796.004 & 161.353 & 0.092 & 33.777568 & -91.058217 & 76.657 \\
\hline 583.5 & 316911 & 2078551.33 & 1557837.624 & 161.595 & 0.092 & 33.780474 & -91.050864 & 76.897 \\
\hline 584 & 316867 & 2080495.19 & 1559285.394 & 161.659 & 0.092 & 33.784489 & -91.044500 & 76.951 \\
\hline 584.5 & 316823 & 2081707.89 & 1561330.514 & 161.967 & 0.095 & 33.790132 & -91.040555 & 77.265 \\
\hline 585 & 316769 & 2082064.59 & 1564171.643 & 161.770 & 0.092 & 33.797946 & -91.039446 & 77.090 \\
\hline 585.5 & 316715 & 2081371.39 & 1567027.643 & 161.789 & 0.092 & 33.805780 & -91.041792 & 77.113 \\
\hline 586 & 316664 & 2080311.00 & 1569648.762 & 161.879 & 0.089 & 33.812963 & -91.045342 & 77.221 \\
\hline 586.5 & 316614 & 2078733.28 & 1571936.326 & 161.908 & 0.095 & 33.819219 & -91.050588 & 77.273 \\
\hline 587 & 316567 & 2077175.86 & 1574126.751 & 161.916 & 0.095 & 33.825208 & -91.055766 & 77.312 \\
\hline 587.5 & 316520 & 2075685.47 & 1576380.927 & 162.326 & 0.095 & 33.831373 & -91.060726 & 77.726 \\
\hline 588 & 316472 & 2074232.54 & 1578720.679 & 162.675 & 0.095 & 33.837774 & -91.065564 & 78.084 \\
\hline 588.5 & 316426 & 2073274.21 & 1581137.251 & 162.802 & 0.098 & 33.844396 & -91.068777 & 78.221 \\
\hline 589 & 316377 & 2072827.55 & 1583862.213 & 162.900 & 0.098 & 33.851875 & -91.070312 & 78.331 \\
\hline 589.5 & 316324 & 2072838.18 & 1586859.745 & 163.106 & 0.098 & 33.860112 & -91.070348 & 78.539 \\
\hline 590 & 316262 & 2073639.35 & 1590256.407 & 163.332 & 0.095 & 33.869461 & -91.067789 & 78.777 \\
\hline 590.5 & 316206 & 2075353.43 & 1592923.486 & 163.570 & 0.098 & 33.876823 & -91.062205 & 79.017 \\
\hline
\end{tabular}




\begin{tabular}{|c|c|c|c|c|c|c|c|c|}
\hline River Mile & $\begin{array}{c}\text { GPS Time } \\
\text { (seconds) }\end{array}$ & $\begin{array}{c}\text { Easting SP } \\
\text { (feet) }\end{array}$ & $\begin{array}{c}\text { Northing SP } \\
\text { (feet) }\end{array}$ & $\begin{array}{l}\text { Elevation } \\
\text { Geoid 03 }\end{array}$ & $\begin{array}{c}\text { Standard } \\
\text { Deviation }\end{array}$ & Latitude & Longitude & $\begin{array}{c}\text { Ellipsoid Ht } \\
\text { U.S. Survey } \\
\text { (feet) }\end{array}$ \\
\hline 591 & 316160 & 2077304.76 & 1594697.504 & 163.429 & 0.098 & 33.881736 & -91.055818 & 78.883 \\
\hline 591.5 & 316119 & 2078867.36 & 1596460.259 & 163.498 & 0.098 & 33.886610 & -91.050710 & 78.999 \\
\hline 592 & 316077 & 2080399.93 & 1598371.596 & 163.543 & 0.102 & 33.891891 & -91.045704 & 78.982 \\
\hline 592.5 & 316030 & 2082320.70 & 1600322.508 & 163.930 & 0.102 & 33.897288 & -91.039419 & 79.404 \\
\hline 593 & 315985 & 2084372.54 & 1601966.49 & 163.944 & 0.098 & 33.901844 & -91.032695 & 79.439 \\
\hline 593.5 & 315938 & 2086252.61 & 1603968.843 & 164.318 & 0.098 & 33.907381 & -91.026544 & 79.769 \\
\hline 594 & 315892 & 2087138.22 & 1606403.57 & 164.222 & 0.102 & 33.914088 & -91.023679 & 79.771 \\
\hline 594.5 & 315783 & 2089753.69 & 1608800.416 & 164.790 & 0.102 & 33.920722 & -91.015112 & 80.229 \\
\hline 595 & 315724 & 2091171.26 & 1611616.906 & 164.981 & 0.102 & 33.928487 & -91.010500 & 80.471 \\
\hline 595.5 & 315650 & 2089731.56 & 1615030.38 & 165.035 & 0.108 & 33.937841 & -91.015321 & 80.505 \\
\hline 596 & 315564 & 2085848.30 & 1617124.269 & 165.213 & 0.105 & 33.943523 & -91.028170 & 80.720 \\
\hline 596.5 & 315516 & 2083360.82 & 1617931.452 & 165.281 & 0.112 & 33.945694 & -91.036389 & 80.741 \\
\hline 597 & 315469 & 2080793.07 & 1618485.591 & 165.436 & 0.108 & 33.947168 & -91.044868 & 80.934 \\
\hline
\end{tabular}




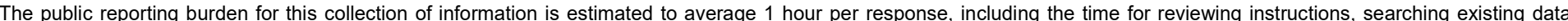

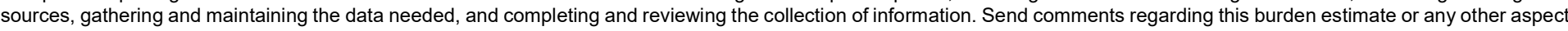

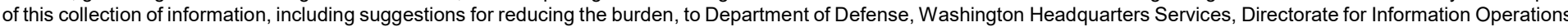

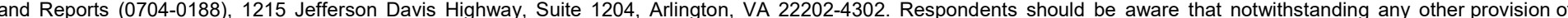
law, no person shall be subject to any penalty for failing to comply with a collection of information if it does not display a currently valid OMB control number.

PLEASE DO NOT RETURN YOUR FORM TO THE ABOVE ADDRESS.

\section{\begin{tabular}{l|l} 
1. REPORT DATE & 2. REPORT TYPE
\end{tabular}}

April 2018

\section{TITLE AND SUBTITLE}

Final Report

Bed-Load and Water Surface Measurements during the 2011 Mississippi River Flood at Vicksburg, Mississippi

\section{DATES COVERED (From - To)}

5a. CONTRACT NUMBER

5b. GRANT NUMBER

5c. PROGRAM ELEMENT NUMBER

5d. PROJECT NUMBER

127672

5e. TASK NUMBER

5f. WORK UNIT NUMBER

8. PERFORMING ORGANIZATION REPORT NUMBER

MRG\&P Report No. 18

10. SPONSOR/MONITOR'S ACRONYM(S)

USACE MVD

11. SPONSOR/MONITOR'S REPORT NUMBER(S)
1400 Walnut Street

Vicksburg, MS 39180

\section{DISTRIBUTION/AVAILABILITY STATEMENT}

Approved for public release; distribution is unlimited.

\section{SUPPLEMENTARY NOTES}

\section{ABSTRACT}

The U.S. Army Engineer Research and Development Center, Coastal and Hydraulics Laboratory, Field Data Collection and Analysis Branch collected bathymetry data and water surface elevations during the 2011 Flood. According to the gage at Vicksburg, MS, the flood peaked at a stage of 57.1 and a flow of 2,310,000 cubic feet per second (cfs) on 17 May 2011, both the highest of record. The reported water surface data include a continuous water surface profile from river mile (RM) 597 to RM 362 and water surface measurements in the vicinity of the Yazoo Backwater levee and Vicksburg, MS. Bathymetry data were also collected and include five sets of surveys, compatible with the Integrated Section Surface Difference over Time Version 2 (ISSDOTv2) method of measuring bed-load transport. These five sets were surveyed at flow rates between 1.6 million and 2.3 million cfs. ISSDOTv2 was used to calculate bed-load transport for the five sets of data. These new bed-load data populate the Lower Mississippi River bed-load rating curve at higher flows and provide a higher level of confidence in the higher-flow portions of the curve. The resulting curve can be a powerful tool for inferring past bed-load transport and predicting future transport.

\section{SUBJECT TERMS}

Bed load-Measurement, Mississippi River, Stream measurements, Vicksburg (Miss.), Water levels

\section{SECURITY CLASSIFICATION OF:}

\begin{tabular}{|l|l|l|}
\hline a. REPORT & b. ABSTRACT & c. THIS PAGE \\
Unclassified & Unclassified & Unclassified \\
& &
\end{tabular}

17. LIMITATION OF ABSTRACT

SAR 18. NUMBER OF
PAGES

52 19a. NAME OF RESPONSIBLE PERSON Ty V. Wamsley

19b. TELEPHONE NUMBER (Include area code) 602-634-5062 